This is an informal report intended primarily for internal or limited external distribution. The opinions and conclusions stated are those of the author and may or may not be those of the laboratory.

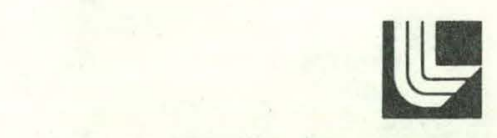

LAWRENCE LIVERMORE LABORATORY

University of California/Livermore, California

\title{
FEASIBILITY OF OBSERVATION OF CAVITY GROWTH \\ BY E.M. SCATTERING (Appendix)
}

(U)

\author{
L. F. Wouters
}

Apri1 1, 1969

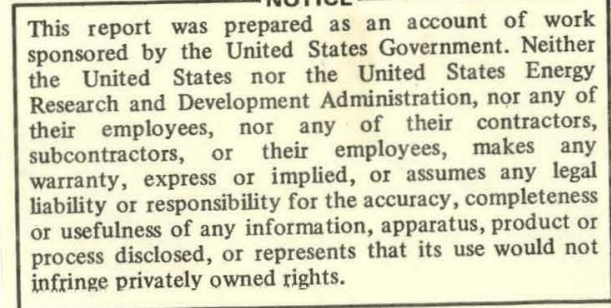




\section{DISCLAIMER}

This report was prepared as an account of work sponsored by an agency of the United States Government. Neither the United States Government nor any agency Thereof, nor any of their employees, makes any warranty, express or implied, or assumes any legal liability or responsibility for the accuracy, completeness, or usefulness of any information, apparatus, product, or process disclosed, or represents that its use would not infringe privately owned rights. Reference herein to any specific commercial product, process, or service by trade name, trademark, manufacturer, or otherwise does not necessarily constitute or imply its endorsement, recommendation, or favoring by the United States Government or any agency thereof. The views and opinions of authors expressed herein do not necessarily state or reflect those of the United States Government or any agency thereof. 


\section{DISCLAIMER}

Portions of this document may be illegible in electronic image products. Images are produced from the best available original document. 


\section{FEASIBILITY OF OBSERVATION OF CAVITY GROWTH \\ BY E.M. SCATTERING \\ APPENDIX \\ L. F. Wouters \\ Apri1 1, 1969}

\section{INTRODUCTION}

The general concept studied here is that of illuminating an underground nuclear explosion cavity with various types of EM energy, and possibly observing the interaction with that growing, conducting spherical body - al1 in the hopes of learning something about the cavity history and the total yield. Here I will present in detail, the line of analysis and its quantitative and qualitative rationales and justifications, with respect to the question of "visibility" only. This leads to the six representative case calculations which were summarized in UCID-16781. I do not here consider the subsequent question of interpretive "quality".

The general idea is illustrated in figure 1 ; the analys is scheme is outlined in Table I and its general results in Table II. These and subsequent figures are reproduced from UCID-16781. This appendix includes: Introduction; Analysis Strategy; Specific Cases
A. Experimental Conditions
P. 21
B. Configuration Criteria and Constraints
P. 30
C. Analysis Descriptions
P. 47
D. The Prototype Cases
P. 98
Tables and Figures
P. 120 
Vocabulary

A number of terms used in these feasibility developments deserve clearer definition:

"Cavity" identifies a hot, conducting, expanding, spherical nuclear explosion cavity. It does not here mean an electromagnetically empty space. This is important, because we are concerned with the fields outside such a spherical conductor - not the ones inside.

"System" will be used to identify the entire configuration of Transmitter, Cavity and Receiver. When suitably. modified, reference is made to a sub-system, such as "receiver system".

"Component", where not otherwise specified, will mean a smal1 portion of a sub-system, such as a filter device, or an electronic amplifier.

"Element" will usually identify a field-producing or a fieldsensing device, such as a loop or an "antenna".

"Transmitter" wi11 identify the complete field generating package, including the connected generating element.

"Generator" will specifically reference that transmitter element.

"Receiver" will identify the complete field measuring package, including the connected sensing element. 
"Sensor" will specifically reference that receiver element.

"Illumination" will indicate the process of coupling generator field energy to the conducting cavity (or directly to the sensor).

"Scattering" will indicate the process of reradiation of e.m. energy by the cavity.

"Signal" (S) identifies the scattered field signal as seen by a sensor.

"Background" (B) identifies the directly-induced transmitter field signal as seen by the sensor. (Note again figure 1)

"Noise" (N) will refer to the variety of extraneous signals generated by other sources, such as UG EMP, Test power, and T \& F systems, strong shock, VLF and MF radio stations, lightning, switching transients, etc.

$\underline{S / B}$ obviously identifies the Signal/Background ratio.

S/N likewise identifies the Signal/Noise ratio.

Finally, I will use MKS units exclusively. 
ANALYSIS STRATEGY

First, certain relevant conditions imposed by established underground test practices are discussed at some length in Section $A$. The chief ones included the adoption of a single prototype explosion model - a $5 \mathrm{kt}$ test at nominal containment depth $(200 \mathrm{~m})$, and the selection of just two representative test media. (A typical alluvium and granite.) The intrinsic electromagnetic properties of these media are characterized by the two parameters:

$$
\begin{aligned}
\text { Dielectric constant: } \cdot \mathrm{K}_{\mathrm{g}} & =9 \text { for both media } \\
\text { Resistivity: } \quad \rho=\frac{1}{\sigma} & =400 \text { ohm-meter for alluvium } \\
\rho & =\frac{1}{\sigma}=10^{4} \text { ohm-meter for granite }
\end{aligned}
$$

A credible model also was needed for the em properties of the explosion cavity. Here it is assumed to contain a high-conductivity plasma $\left(\rho=\frac{1}{\sigma}=10^{-3}\right.$ ohm-meter or lower). The adopted cavity growth curve is shown in figure 2 , along with some other related variables of significance to the study. The final radius is $\sim 28 \mathrm{~m}$. The validity of such an "infinitely conductive" sphere model is also discussed in Section A.

Next, in considering the electromagnetic problem itself, it was immediately evident that we would need appropriate criteria and constraints by which to eliminate redundant and "low-grade" possibilities, and to concentrate attention on optimum typical systems and on systems exhibiting singular features. These criteria fell into three categories:

Frequency Domains - Generator Coupling - Sensor Configurations Their logic and definition are discussed extensively in Section B. 
A broad economic and physical constraint which was imposed early in the study, was that the major system elements would be located on or near the earth's surface. Any significant upward or downward departure therefrom could easily involve a higher price tag than that of the test facility itself!

Another early conceptual aspect concerned the appropriate frequency domains. There were initially no restrictions to this, other than the well known, severe propagation cutoff in alluvium, above the VLF regime.

An important feature in this respect, is the resonance mechanism for electromagnetic fields outside a conducting sphere. The lowest order mode is defined by $x_{\mathrm{g}}=7.3 \mathrm{R}$ which corresponds to a frequency $\sim 600 \mathrm{kHz}$. It would be detectable in granite only. As a result of these and other constraints, three specific frequency domains were chosen:

(1) D.C. - artificial and natural fields

(2) V.L.F.- specifically $\omega=2 \times 10^{4}$

(3) M.F. - specifically $\omega=2 \times 10^{6}$.

Self-evidentiy, one avenue for overcoming some of the circumstantial problems could lie simply in "more power". To prevent the analys is from becoming an exercise in field escalation, as well as to provide realistic case comparisons, certain hardware limitations were invoked on the generation of artificial environments:

(1) Maximum power input - $100 \mathrm{~kW}$

(2) Maximum generator voltage - $10^{4}$ Volts 
Ultimately, our considerations identified three broad forms of applied field distributions, in association with the most feasible hardware systems:

(1) Earth conduction

(2) Quasi-static magnetic fields

(3) EM radiation fields

This array of conditions and alternatives would still seem to lead to a large number of permutations. However, only a limited number of possibilities survived the tests of self-consistency and selection criteria. These qualitatively meaningful combinations include:

Earth conduction schemes - DC and VLF in alluvium only. Quasi-static magnetic fields - VLF in both alluvium and granite.. EM radiation fields - MF in granite only.

\section{System Evaluation Plan}

Further consideration could not avoid specific hardware issues, and we continued by taking a closer look at the "signal paths".

Figure 1 indicates that there are two general routes for energy transfer of interest to us: The indirect, signal path from generator to cavity to sensor, and the direct, background path from generator to sensor. The indirect path is distinguished by attenuation through the medium, as well as by the equivalent of an additional solid angle. It would carry the cavity dimension information. of interest, as an amplitude (and possibly "phase") signature. 
Thus there is an intrinsically disadvantageous signal/background situation, as well as the more conventional signal/noise problem. The feasibility of the various system options will therefore also depend on the opportunities which they present for redressing those major shortcomings. In part, this can be measured by two dominant hardware optimization criteria:

(1) Generator configurations which maximize the EM energy coupled to the conducting cavity surface.

(2) Sensor geometries which maximize the signal/background $\operatorname{ratio}(S / B)$.

In these evaluations, I have otherwise neglected the air-earth interface; in effect, this is equivalent to burying both the generator and sensor elements, but still assuming the "worst-case" background.

Sets of analytic models were constructed, corresponding to the "meaningful combinations" outlined above, for the purposes of calculating the representative systems, and of optimizing the selection of such case parameters. In the interests of not getting mired here in the swamps of exact solutions (of Maxwe11's equations), a catalogue of pertinent calculational formulae was collected or derived. Some of these are "exact" and some are approximations. They are discussed in Section C. A particularly useful concept is the "Laplace equivalence" principle, which identifies the commonality of solutions for identical field problems, irrespective of the field vehicle. Finally, the specific 
case calculations are described in Section $D$. Those results are believed to be good to better than an order of magnitude and in some cases, down to a factor of two. (N.B. on "idealizations".) Everything is done in mks units.

An exact, detailed, computerized, general solution of Maxwell's equations has also been written for the "figure 1 " problem, and it is in use. Some of its initial results have guided certain choices and avenues discussed here. Its development and "mid-course" results are being separately reported. It has the further ultimate purposes of:

locating possible, more sophisticated, "surprises", describing quantitatively the (exterior) resonance case, and providing a precise basis for experimental design and interpretation, if one elects to "try". 


\section{THE SPECIFIC EVALUATION CASES (Section D)}

The prima fascie purpose for numerical evaluation is to furnish quantitative measures by which various classes of schemes may be "eliminated", or at least be found to be clearly deficient relative to others. The principle evaluation numbers here turn out to be the scattered signal amplitude itself $(S)$, and the ratio $S / B$ (or, in one or two cases, $S / N)$.

We will see that this is a deceptive cause. For one thing, the circumivention possibilities are so numerous, and potentially so powerful (in some cases), that relatively poor S/B numbers are not necessarily fatal. I have therefore elected to "turn the problem around", by specifying a "Nulling factor" $\left(N_{f}\right)$. It is essentially the inverse of $S / B$, and it measures "how hard one has to work" in order to redress that number to unity (or better).

\section{The Conduction Current Schemes ( $D C$ and VLF)}

This configuration category is illustrated in figure 3 . The basic idea is to illuminate the explosion region with a two-dimensional current field. (Thereby removing one order of divergence in energy coupling.) The expanding conducting sphere increasingly distorts the current distribution, producing a locally higher current density. It then behaves as a magnetic field generator, whose field strength may be related to the cavity growth. 
The general concept is limited to alluvium, by the necessity of obtaining substantial current densities at reasonable electrode voltages, and it is Timited to D.C. and VLF frequencies by virtue of phase/dimension and skin-depth penetration considerations.

The physical requirements may be envisioned by summarizing certain specification features of the generator and sensor elements, which are more or less common to both cases.

Typical Hardware - The generator element would amount to a pair of "earth contacts". As a prototype specification, I use a contact $200 \mathrm{~m}$ long, separated from its opposite number by $400 \mathrm{~m}$. These could be constructed by surface burial of $2 \mathrm{ft}$ diameter, perforated (and corrugated) galvanized, sheet steel culvert pipe, $660 \mathrm{ft}$ long each. This would be periodically filled with a saturated $\mathrm{CuSO}_{4}$ solution $(30,000$ gallon per fill). Alternatively, 11 vertical, $1 \mathrm{ft}$ diameter, perforated steel casings could be driven down $60 \mathrm{ft}$ along each contact line. They would be similarly treated with $\mathrm{CuSO}_{4}$ solution.

The power supply' connection must have as low an inductance as feasible (in the VLF case), and this could be ideally provided by means of two large triangular copper sheets (!). Their apex would connect to the power source, which would be located at the center of the array. More practically, one could probably get by using divergent, flat copper strips, $1 \mathrm{ft}$ wide, to each vertical casing (or equivalent culvert point). 
The sensor is not "trivial" either. In the DC case, it consists of a 5000 turn loop of 1/8" diameter stranded wire, $30 \mathrm{ft}$ in diameter, and erected in a vertical plane immediately above the shot point. (It would preferably be buried in a "slot" trench - but that may be going too far.)

The winding would be about $1 \mathrm{ft}$ square in cross section, probably plastic-cast, and weighing about 25 tons. It would probably not have a "simple" winding pattern.

\section{D.C. Excitation Case}

The current field is produced by passage of a direct current through the earth. Since the sensor is chosen to be a rate-of-change device, in principle it will respond only to the cavity signal, and should not "see" the generator. The feasibility estimate includes the following calcurational sequence as major steps:

The field power requirement (and generator element specification).

The local current distribution (by the Laplace principle).

The current moment of the cavity (assuming the situation to be that of an infinitely conducting sphere immersed in a once-uniform current field).

The magnetic field distribution of the current moment as a dipole.

The induced sensor signal (in which the cavity history appears in the form of a characteristic rate of change $\alpha_{c}=\frac{1}{R} \frac{\partial R}{\partial t}$ - the "cavity alpha"). 
As just indicated, S/B is not an appropriate parameter for this particular case. However, because it is an intrinsically real-time scheme (with no shift in frequency domain by virtue of an R.F. carrier), it is very susceptible to noise contributions. (As the magnetic bubble was once thought to be.) Overwhelming noise sources include:

Power Supply Ripple

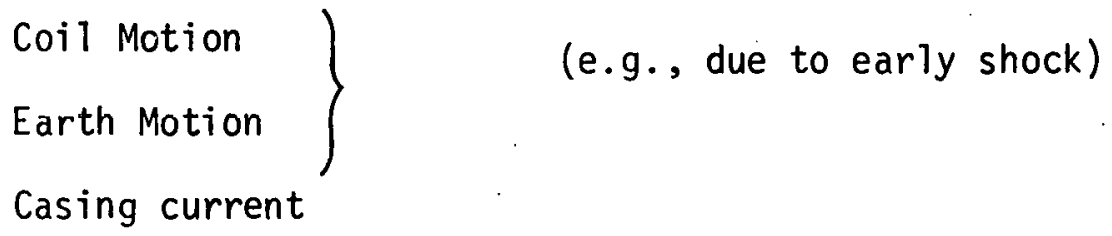

\section{VLF Excitation Case}

Now we impose a VLF voltage on the electrodes in order to produce the current field. This is essentially a carrier scheme, so that the noise considerations are replaced by background estimates. The wavelength is chosen long enough to permit quantitative consideration on a quasi-static basis $\left(x_{g} \sim 5000 \mathrm{~m}\right)$. Thus, essentially the same calculational sequence applies, as was outlined for the previous D.C. case. One difference is in the (sensor) rate-of-change definition, which is now $\omega$ instead of $\alpha$. The background estimate adds a few steps to the sequence:

The surface background magnetic field (due to the diffuse earth current distribution).

The induced sensor background signal.

The $S / B$ ratio and $N_{f}$. 
The VLF Magnetic Field Schemes (Figures 4, 5, 6)

The basic idea here is to illuminate the explosion region using a VLF Magnetic Field, whose distribution and interaction may be treated as quasi-static. * The expanding spherical conductor intercepts this field, and by self-induction, excludes it from the cavity. This can be modeled to first order, as an oppositely oriented magnetic dipole moment, whose field strength may be related to the cavity growth.

This is perhaps latently the broadest class of schemes. It includes the general idea of "going out there with some loops and some borrowed R.F. gear to see what happens". At the very least, I hope that this study will dissuade people from the folly of that particular empiric approach. It constituted the class which was most difficult of optimization and selection - yet, it also has turned out to contain the less attractive schemes, as we can see from Table II.

The three schemes of figures 4,5 and 6 are optimum variants, in some way, of configurations using resonant loop generators and resonant loop sensors. Here, the basic concept is to induce surface currents on the conducting cavity by means of VLF magnetic field illumination, and to observe the re-radiated flux. This "return" field strength would be presumed to be related to cavity size. The instrumentation scheme has to sort it out of the "direct background".

\footnotetext{
"Quasi-static" here corresponds to wavelengths large compared to major configuration distances (dob, cavity size, etc.).
} 
The cavity rate-of-growth is sufficiently smaller than the frequency $\left(\alpha_{c}<\omega\right)$, such that the R.F. interaction may also be evaluated on a quasi-static geometrical basis - that is, point-by-point along the expansion history.

An early result of a "universal" optimization exercise was that, for a given VLF generator power and voltage, maximum coupling was attained with the cavity by using a (100p) generator of dimensions comparable to the depth of burial $(\sim 200 \mathrm{~m})$ : This turns out to be a relatively inviolate criterion, with steep fall-off penalties for significantly smaller (or larger) choices. It does have one side benefit - such a loop is always much larger than the subsidence crater, so that the physical investment is easier to salvage!

Other optimization features involve: generator placement as near as possible to the cavity (hence immediately overhead), sensor orientation orthogonal to generator ("crossed loops") in the interests of best $S / B$, and finally, sensor placement for optimum scattered field vector linkage (which is not necessarily directly overhead).

Typical Hardware - The VLF generator element would be a very large, two-turn loop, $200 \mathrm{~m}$ in radius (1300 ft across!). (One could probably do almost as well with three turns, $100 \mathrm{~m}$ in radius.) The conductor would consist of $4^{\prime \prime} \times 7^{\prime \prime}$ copper bus (or equivalent area) and this coil would weigh around 50 tons. It would probably be supported by means of insulated "Lord" shock mounts fastened to a circular array of reinforced concrete piers. 
The VLF sensor element would be somewhat less imposing, consisting of an 80-turn 10op, $30 \mathrm{ft}$ in diameter, and again erected in a vertical plane. Using 1/8" diameter stranded wire, it would only weigh about one ton, having about a 1 " square section.

\section{Axial Illumination - Crossed Loops (figure 4)}

This scheme exactly epitomizes the hardware just described. The "magic" distance (for the Sensor element) is $\ell=\sqrt{2} \mathrm{~d}$. The induced magnetic dipole moment of the cavity is vertical, and the sensor is thus located in an (orthogonal) fringe field as shown. The feasibility estimate here includes the following calculational sequence as major steps:

Signal: Criterial specification of generator and sensor loops. The cavity field description (Q.S. axial loop expression). The induced cavity dipole moment (again as a perfectly diamagnetic sphere in a once-uniform field).

The magnetic field distribution due to that dipole moment.

The induced sensor signal.

The S/B ratio goes to infinity here for an ideal physical geometry. The reason for this, is that the small radial components of the background field generate equal and opposite contributions in the two halves of the loop area. (Above and below its axis.) This presupposes precise alignment 
of the sensor axis in the equatorial plane of the transmitter 1oop, of course. The required "perfection" of this geometry is measured by calculating the maximum possible induced signal (i.e., $90^{\circ}$ rotation):

The direct background field at the sensor.

The induced background signal (S).

The $S / B$ ratio and overall $N_{f}$.

\section{Axial Illumination - Concentric Loops (figure 5)}

The difficulty of generating a. "comfortable" sensor signal together with a reasonable $S / B$ ratio, provoked the idea of abandoning $S / B$ as a constraint, and simply going for the maximum possible sensor-cavity, coupling. The severely adverse background penalty would then be recouped by excessive cleverness and ingenuity! Obviously, maximum sensor-cavity coupling is achieved in exactly the same way as maximum generator-cavity coupling. Since we're stuck on the surface, this also leads at once to maximum generator-sensor coupling. Hence, we obtain the mutual axis scheme of figure 5 .

A way out of the dilemma is the singular concept of a quadrupole sensor loop. Basically, it consists of two identical but separated loops, located coaxially and symmetrically, with respect to the generator loop, and connected "back-to-back". In an idealized situation, no net background signal would be generated. Yet, because of the gradient in the fringe field of the cavity dipole, there would be a net signal of cavity origin. 
The hardware elements assumed here are identical to the first case except that the sensor elements consist of two of those loops, arranged and connected as just discussed and illustrated.

The signal calculation sequence is basically identical to the previous case, down to the induced sensor signal step. Here this becomes:

Expression of the quadrupole-coupled field gradient component (by a first-order space derivative).

The effective field rate-of-change expression.

The induced sensor signal.

For this case, the background calculation amounts to an estimate of the "perfection" to which the pair of loops must be a quadrupole -i.e., . the ratio of the residual dipole moment to quadrupole moment. The generator-induced voltage in each loop is estimated by the same routine as was the background for the previous (VLF) case.

Incidentally, a "real" quadrupole pair would probably be constructed quite differently than is assumed in this ideal model.

\section{Equatorial Illumination - Crossed Loops (figure 6)}

This is included principally because it is geometrically the "mirror image" of the first case (figure 4). Pragmatic limitations drastically reduce the permissible size of the generator element. (Its plane is vertical.) The induced magnetic dipole moment of the cavity is now horizontal, but again we place the sensor at the "magic" distance in the fringe field $\left(\ell=\frac{d}{\sqrt{2}}\right)$. 
In principle, $S / B$ is again infinite here for an ideal physical geometry. Here the reason for this, is that the plane of the sensor loop is in the axis of the generator loop; if the sensor is truly symmetric and well aligned, there is no net coupling to the generator field. Again, I estimate the necessary "perfection" of such symmetry, by calculating the maximum possible induced signal (i.e., $90^{\circ}$ rotation), thereby defining the overall nulling factor.

The specific hardware elements assumed for this evaluation include a sensor loop identical to the first case, but a redefined generator loop, now subject to a vertical size criterion (rather than optimum coupling). We will see that this loses us a factor of $\sim 100$ in system effectiveness.

The feasibility estimates are identical in procedure as for the first VLF case.

Here also a "real" sensor coil would probably have a fairly complex design, involving such features as interlaced and carefully matched winding layers. 
The M.F. Radiation Scheme (Similar to figure 6)

This is a relatively straightforward, "radar" scheme; the basic idea is to illuminate the cavity with radiation of wavelengths comparable to the cavity dimension, and to observe the re-radiated energy. This should have cavity growth written into it, in respect to amplitude, phase and frequency-dependent anomalies. (Exterior resonance.)

This application is limited to granite, because the absorption in alluvium at the required frequencies is completely unacceptable. (e.g., $e^{-15}$ per path.)

For estimation purposes here, a wavelength sufficiently removed from the lowest spherical mode was chosen, such that the coupling problem could still be credibly dealt with by quasistatic field models, but also such that the path propagation was predominantly radiative. Several "primitive" generator-sensor alternatives were estimated, but, as would be expected, the fixed power criterion resulted in essentially identical illumination and scattered fields. So we count this as a single case.

The feasibility estimate follows this sequence:

Generator dipole moment (from power criterion).

Cavity illumination field definition.

The induced cavity dipole moment.

The cavity dipole radiation field.

The induced sensor signal. 
Again, the background was estimated on a "worst-case" basis, to specify the required suppression factor:

The direct background field (at sensor).

The induced background signal.

The $S / B$ ratio and $N_{f}$.

Typical hardware - There are a variety of "standard practice" means to satisfy the generator-sensor requirements here - some of them "off-theshelf" even. For instance, a typical generator element would consist of a 10-turn loop, $10 \mathrm{ft}$ in diameter, in the vertical plane. The conductor would be $\frac{1}{2} "$ copper pipe, probably water-cooled. The sensor element could be a ferrite-loaded resonant loop, such as is used in standard LF/MF direction finders. In practice, the MF-granite scheme would doubtlessly be engineered with much more sophistication than is indicated by this "primitive" model. 
A. THE EXPERIMENTAL CONDITIONS

For our purposes, certain circumstances and consequences of environment and of test explosion are considered as not "open to appeal", from the viewpoint of experimental design. These include such matters as the electromagnetically significant features of the early explosion cavity, the EM properties of the principal test media, the constraints imposed by the broad restriction to surface installations. For instance, this last restriction has the immediate qualitative consequence that energy transferred along the scattering "signal" path suffers significant attenuation, whereas that transferred along the surface, "background" path, does not. Certain ingenious EM devices (shields, diffusers, field nulling) were devised which could ameliorate this particular handicap in a worthwhile way. (These are not discussed further here.)

\section{The Prototype Test Explosion}

The choice of a single, prototype explosion case was motivated in the interests of ready comparison of different configurations, and again, by a desire to remove issues extraneous to the basic concept. The selected case is a $5 \mathrm{kt}$ yield at "nominal" containment depth, as defined by the $350 \mathrm{~W}^{1 / 3}$ criterion. In mks units this translates to a DOB:

$d=115 W^{1 / 3} \simeq 200$ meters. $\left(W^{1 / 3}=1.71\right)$

The EM scattering scheme is addressed to the broad problem of total yield determination, albeit indirectly. Actually, the high yield regime is the one of principal concern in this respect. However, some brief extrapolations of the models discussed below, suggested that "seeing" 
a high-yield (megatori) cavity at its containment depth would involve an even higher order of difficulty. What's more, the basic steps towards greater feasibility would probably require a substantial redirection of test program investments to locations of relatively special media, such as large, competent masses of uniform, dry granite. In any case, the likely experimental investment at high yields, would indicate that one should first execute at least one successful low-yield experiment at a readily available site (e.g., Area 18). Also, if there is any chance of "seeing something" in the more conventional NTS tests in alluvium, it would necessarily lie in the nominal yield domain. This was the basic rationale for the $5 \mathrm{kt}$ prototype choice.

\section{The Earth Medium}

In the continuing interests of pursuing electromagnetic feasibility studies, the considerations were restricted to two representative geologic cases:

(1) Alluvium of low specific resistivity $\left(\frac{1}{\sigma}\right) \sim 400 \mathrm{ohm}-\mathrm{meter}$, and $\kappa_{g} \sim 9$. This is also called the high-conductivity or "wet" case.

(2) Granite of high specific resistivity $\left(\frac{1}{\sigma}\right) \sim 10^{4}$ ohm-meter, and $\mathrm{k}_{\mathrm{g}} \sim 9$. This is also called the low-conductivity or "dry-rock" case.

It is well known that NTS alluvia and tuffs exhibit resistivities as low as 20 ohm-meter; perhaps a value of 100 ohm-meters is a better average of in-situ resistivity measurements. However, our UG EMP observations suggest that a higher mean value, better satisfies such 
theoretical models as we have to date. Considerations of dry-rock systems (in granite, basalt, etc.) are much less sensitive to specific choice of the resistivity number. For the situations considered here, the rock conduction effects are trivial.

Finally the choice of $\mathrm{K}_{\mathrm{g}}$ is also relatively insensitive, since it enters predominantly as $\sqrt{k_{g}}$ in the effective propagation velocities. Again. the indicated value arises from fitting to UG EMP data. Throughout, the earth medium is considered to be electrically uniform in the regions of interest.

In the consequence of these broad features of the native media, the following numbers apply:

\section{ALLUVIUM $\quad$ GRANITE}

Dielectric Constant 9 9

Velocity of Propagation (limit value)

$\begin{array}{rcc}10^{8} & 10^{8} & \mathrm{~m} / \mathrm{s} \\ .0025 & 10^{-4} & \mathrm{mho} / \mathrm{m} \\ 400 & 10^{4} & \text { ohm-m }\end{array}$

EM Absorption Length

$$
\delta=200 \quad \gamma_{\mathrm{g}}=160 \mathrm{~m}
$$

at $\delta \sim 2 \times 10^{4}$ in MF-HF domain

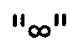
in VLF domain 


\section{The Air-Earth Interface}

Another invariant physical feature of electromagnetic significance is the dielectric interface at the earth's surface. Aside from the economic constraint which "freezes" experiments to its vicinity, it may also do strange things to our EM field distributions - perhaps not beneficially so.

of course, this will depend on the frequency domain of interest. Inasmuch as we assume $\mu=1$, then quasi-static magnetic field distributions will be unaffected thereby. Further along, such field descriptions will be associated with the DC and VLF domains, and to first-order, I will neglect interface effects there. But it is a valid reservation.

At higher frequencies (the MF radiation domain) in granite, there certainly will be significant interface effects, such as the equivalents of refraction and total internal reflection. We can get around this conceptually by assuming that the transmitter and receiver elements are buried "sufficiently" below the surface. In terms of physical reality, one can conjure a legion of ingenious devices to circumvent (or even, to opportune) the problem, since the element dimensions are physically modest. For estimation purposes here, I assume that air is granite(!).

\section{The Plasma Cavity Model}

Another initial study assumption was the electromagnetic representation of the explosion cavity as an expanding, infinitely-conducting sphere. Related to this, it must be emphasized that the EM considerations are 
addressed entirely to interaction mechanisms involving EM fields exterior to the growing (or ultimately stabilized) cavity. Implicitly, the applied fields must induce surface ("eddy") currents on that sphere - or perhaps more accurately, in the hot plasma just beneath the instantaneous solid matter interface.

The treatment of this model will involve another basic interaction assumption: That a sufficiently accurate electromagnetic description obtains, by calculating the field interaction with the instantaneous cavity as if it were dimensionally stagnant - i.e., a quasi-static geometric model. (I have also indicated the use of quasi-static field descriptions. This is a notice not to confuse them.)

The extent to which this is an acceptable electromagnetic model, may be judged from the following explosion plasma description.

As soon as the thermal energy "breaks out" of the reacting device, the gas in the test canister becomes essentially completely ionized. During the early expansion phases, the plasma temperature is in the tens of eV, and it is customary to analyze cavity plasma problems in terms of numbers of ionized electrons per atom. This will range from the order of 10 down to 1 , as the cavity expands. In this domain, it is difficult to speak of conductivity or resistivity in the conventional sense. For example, when the plasma condition corresponds to one free electron per atom, it still contains about $10 \%$ of the yield energy. The electron density is $\sim 10^{26} / \mathrm{m}^{3}$ and if we simply use $\sigma=\mathrm{Ne} \mu$, we find $\sigma \sim 10^{5} \mathrm{mho} / \mathrm{m} ! !$ The corresponding skin depth at $3 \mathrm{khz}\left(\omega \sim 2 \times 10^{4}\right)$ would be $\delta=\sqrt{\frac{2}{\mu_{0} \sigma \omega}} \sim .1 \mathrm{~m}$. Evidently these may be somewhat artificial numbers. 
If we think of $\sigma$ in terms of electromagnetic attenuation - i.e., in terms of $\sigma E$ in Maxwell's equations - it takes on equivalent values of $10^{3}$ down to $10(\mathrm{mho} / \mathrm{m})$. Sea water has a value of 4 , and the boundary conductivity of the conduction zone in the classicial atmospheric EMP problem is $\sim 10^{-4}(!)$. Note that our earth media lie in the value range $10^{-2}$ to $10^{-4}$.

As the cavity expansion proceeds further, and we approach the "knee", the temperature falls nominally below the ionization thresholds, although its "upper tail" still preserves a significant electron density. At $\sim 10 \mathrm{eV}$ the electron density is still $\sim 1$ per atom, and even at $2 \mathrm{eV}$, it is $\sim 10^{-4}$ per atom, corresponding to a conductivity of order $1 \mathrm{mho} / \mathrm{m}$. It cannot yet support large fields. Only in the final phases of expansion, does the gas become electromagnetically transparent. Quasistatically, the imposed field lines would diffuse back in. It thus appears that a "perfect conductor" is a good starting approximation here.

It is evident that the cold cavity (prior to collapse) may also exhibit interesting electromagnetic features, since it now appears as an. "empty" spherical cavity in a (poorly-conducting) dielectric. The granite case in fact corresponds to a standard textbook problem. Notice that we have implied in this model, that the cavity must effectively "switch" from one state to the other rather quickly and discontinuously. (The lines "snap" inward.) It is not a "poor conductor" very long. (This neglects ionization due to residual gaseous radioactive products, and due to the radioactive products trapped in the cavity surface "glass".) 
Cavity Growth - In the "scattering" arithmetic, the characteristics of chief interest will be the radius (or volume) growth function $R(t)$, its rate of change $\frac{\partial R}{\partial t}$, and the final stabilized radius. For the prototype $5 \mathrm{kt}$ explosion, a typical expansion history is depicted in figure 2.

Certain useful parameters are also plotted there, including:

The "Cavity Alpha": This is defined as

$$
\alpha_{c}=\frac{1}{R} \frac{\partial R}{\partial t}
$$

Note that this has a maximum value of $210^{3} \mathrm{sec}^{-1}$. It is not yield sensitive, incidentally.

The $k$ ratio:

$$
k=\frac{R}{d}
$$

At final cavity radius, it has the nominal maximum value of $k_{m}=\frac{50}{350}=.14$. (Most of the feasibility expressions turn out to be conveniently expressed in this language.) Then $k_{m}^{3} \simeq 3 \times 10^{-3}$.

The initial part of the expansion converges to a final radius corresponding to infinite dob, and a somewhat smaller $k$ value. For estimates depending on $k^{3}$ only, I will use a conservative value: $k^{3} \simeq 10^{-3}$. This also compensates in part for "early" plasma/field "collapse".

The cavity volume (and $k^{3}$ ). This exhibits a more noticeable "knee", which may find fiducial usefulness.

The final radius. This is also defined by:

$$
R_{C}=16 W^{1 / 3} \text { (meters) }
$$

which is about $28 \mathrm{~m}$ here. 
Certain other "final condition" features may be of interest to us: The "cracking" limit ( $49 \mathrm{~W}^{1 / 3}$ meters), and the probable crater diameter, which is taken as somewhat larger than the stabilized final cavity diameter ( $\sim 35 \mathrm{~W}^{1 / 3}$ meters).

Strong Shock. An early hydrodynamic feature of an underground explosion, is the shock wave which runs out ahead of cavity expansion, essentially at the speed of sound in the medium. For our purpose here, this major environmental perturbation precedes the cavity growth phenomena, and concerns us in at least two ways:

(1) Generation of early false signals. The magnitude of these must depend on the extent to which a strong shock perturbs the electromagnetic properties of the medium, notably in $\kappa_{g}$ and $\sigma$, and by possible piezoelectricity. This problem is not understood at present, even poorly.

(2) Pre-cursor ground motion. Here the principal problems are related to:

Disturbance in energy injection processes (especially in earthconduction methods).

Disturbance in local magnetic field (particularly in real-time sensing schemes).

False signals due to motion of transmitter and receiver elements.

Physical damage to equipment.

While the last two "physical" aspects may not seem (superficially) serious, they take on a different meaning when one contemplates surrounding the test area with a $200 \mathrm{~m}$ loop of copper bus-bar weighing many tons. 
These possible strong-shock complications are quantitatively disregarded in this paper. They may prove to introduce severe experimental and interpretational barriers. However, I felt that the feasibility of the basic schemes should be considered first, assuming the medium to behave post-shock as if it were electromagnetically still in the preshock state. Those schemes which survive that test might well be easier and more specific to deal with, in respect to shock considerations.

Cracking. Contemporary explosion models attribute cracking in the medium outside the cavity, both to early strong shock effects (pulverizing and shearing), and to late expansion forces. (Quasi-static violation of the shear strengths.) Again the degree of electromagnetic perturbation would probably depend on the nature of the medium, and on the type of EM energy injection. Thus a conductive technique in alluvium might be seriously interfered with, whereas a radiative technique in granite might hardly be affected.

I have elected to neglect this intractable feature also, in pursuing these feasibility studies, well recognizing that it may all turn out to be a good way to investigate cracking! 
B. CONFIGURATION CRITERIA AND CONSTRAINTS

In an initial "back-of-the-envelope" survey, several dominant problem aspects emerged. First, many possible and distinct arrangements could test the idea of "illumination scattering" in the VLF-MF regimes. A criterial structure was desirable to "filter" these out. Second, an "exact" Maxwellian calculation for each "survivor" would be a challenging (if not enormous) undertaking. Some intermediate framework of approximations should be adopted for further selection. Third, certain performance characteristics would dominant technical "feasibility":

The EM energy delivered to the cavity.

The "scattered" signal amplitude.

The S/B ratio.

Fourth, the electromagnetic properties of the environments imposed strong biases related to the practical frequency domains, and to the means of energy transfer.

In considering the two significant routes by which RF energy could reach the sensors from the generator, it would appear that the situation would be reciprocal: In principal, a description could be written which would be invariant to the exchange of those elements. This reciprocity did appear somewhat indirectly in the behavior of signal amplitude and S/B. Systems which showed good S/B ratios tended to have low sensitivity; high-sensitivity systems tended to have poor S/B ratios; and these groups belonged to "mirror-image". geometries. 
There were a variety of pragmatic considerations which pre-empted any such simplistic analytic approaches, and which quickly established two broad optimization requirements:

(1) Transmitter schemes which would deliver the maximum energy to the conducting cavity. This was also a good idea on broader grounds of "noise" competition from other EM sources, as will be illustrated.

(2) Receiver schemes which would maximize the S/B ratio while maintaining an acceptable sensitivity to the scattered signal. Tradeoffs have appeared here, which could be further exploited.

Three first-level categories of qualitative "constraints and criteria" were established:

Frequency Domain

Generator/Cavity Coupling

Cavity/Sensor Coupling (and S/B aspects) 
Frequency Domain Considerations - A "Historical" Categorization.

The various possibilities encompass three source frequency regimes:

(1) "D.C." or steady-state current excitation.

(2) V.L.F. or "non-resonant" schemes (1 kHz to $20 \mathrm{kHz})$.

(3) M.F. or "resonant" illumination (100 kHz to $2 \mathrm{mHz}$ ).

These categories are bounded by both pragmatic and analytic corisiderations which find some correspondence to one another.

"Evolution". The original R.F. scattering concept which initiated this study was devised about three years ago. It involved VLF illumination of the cavity region in alluvium. The EM frequencies then considered, were of order 1 to $5 \mathrm{kHz}$. After a generally negative sequence of "hardware" ideas, the possibility of observing the external "resonance" of a conducting sphere was explored. (That resonance was "we11-known" from other studies - note, for instance, Stratton, p. 555.) It was at once apparent that for nominal yield cavities, this involved significantly higher environmental frequencies $(2500 \mathrm{kHz})$, whereas the classical "skin depth" attenuation (or "EM $m f p "$ ) is very short in alluvium at those frequencies $(210 \mathrm{~m})$. The two underground energy paths generally would incur extremely high overall attenuations (e.g., $\mathrm{ve}^{-40}$ ), whereas the background path over the surface would be virtually unattenuated.

At about this point, we were reminded of the H.F. transparency of granite. (Note UCID 15437, Part 2.) Such an environment would greatly improve the feasibility of the resonance scheme, and the detailed "exact solution" program was launched. An early result of this effort is that 
the resonance should indeed be visible as an amplitude and phase anomaly, dependent also on relative orientation and distance. One could then perhaps consider that a workable "Cavity Growth History" scheme could be developed for nuclear tests in granite.

Finally, the (paper) behavior of some of the example VLF schemes suggested that extremely low frequencies - in fact "D.C." - might be explored in an interesting way for alluvium. In such case, the signal rates-of-change become entirely determined by cavity growth. The artificial environment has an effect analogous to the earth's magnetic field, although the experimental embodiment would be quite different. While these recent ideas have not been encouraging, the D.C. example well illustrates, by contrast, the advantages of using an R.F. intelligence carrier which removes the information from a "noisy" domain. Domain Choice Factors. That brief history implicitly indicates some of the chief criteria which define the preferable frequency domains:

a. Power frequencies and their low-order harmonics. Here the Continental U.S. environment is electromagnetically well-polluted. This effectively excludes the spectrum from about $30 \mathrm{hz}$ to about $720 \mathrm{hz}$. This is one criterion for distinguishing classes (1) and (2).

b. The Electromagnetic mean-free-path (or Diffusion length). These amount to the same thing pragmatically, in that they define the distance in which a planar field attenuates by $e^{-1}$. 
In alluvium (400 ohm-m), this is defined by a "skin depth":

$$
\delta=\sqrt{\frac{2}{\mu_{0} \sigma \omega}} \text { meters }
$$

There are distinct restrictions on the validity of this representation; we can use $i t$, as long as $\delta<x_{g}$, where $x_{g}=\frac{v_{g}}{\omega}=\frac{c}{\sqrt{\kappa_{g}} \omega}$.

In granite $\left(10^{4} \mathrm{ohm}-\mathrm{m}\right)$, we may use instead a frequency-independent absorption coefficient:

$$
\frac{1}{\gamma}=\frac{\sigma}{2} \sqrt{\frac{\mu_{0}}{\kappa_{g} \kappa_{0}}}
$$

Now this is valid only for $\gamma>x_{g}$. Since $\gamma \sim 160 \mathrm{~m}$, this description applies to the MF-HF class (3). In the VLF regime, the absorption is essentially negligible in granite. $(\delta \rightarrow \infty)$.

For intermediate frequencies and conductivities, the field solutions involve a transition from one limiting case to another. I have artfully contrived to avoid such situations in this initial study. The Maxwell code includes this business correctly.

The absorption factors enter into the field descriptions as exponential terms, of course:

$$
\begin{aligned}
& \mathrm{e}^{-x / \delta} \text { in Alluvium and for VLF } \\
& \mathrm{e}^{-x / \gamma} \text { in Granite and for MF }
\end{aligned}
$$

(where $x$ is the total path distance). 
If. we set $\delta \sim d$ (the dob) as an upper limit for the non-resonant VLF category in alluvium, we find:

$$
\omega=\frac{2 W^{-2 / 3}}{\mu_{0} \sigma d_{0}^{2}}
$$

where $W$ is in $k t$, and $d_{0}=115 \mathrm{~m}$. Later on, we will fix $W$ at $5 \mathrm{kt}$, so that $\mathrm{d}=200 \mathrm{~m}$, and $\omega \simeq 2 \times 10^{4}$ or $3 \mathrm{kHz}$. Also $x_{\mathrm{g}}=5 \times 10^{3} \mathrm{~m}$. Obviously, we could probably accept somewhat more attenuation and go to a higher frequency, up to say $12 \mathrm{kHz}$, since $\frac{1}{\delta} \propto \sqrt{\nu}$.

Note that (fortuitousiy) $\gamma \sim d$ for this prototype explosion in granite (in the MF domain).

c. The Quasi-Static vs. Plane-Wave Approximations. Speaking in terms of a dipole field approximation, I have tried to also avoid the regimes of more complex problems in which the various pairs of terms are competitive in field description - as quasistatic vs. inductive and inductive vs. radiative. (In fact, I have almost entirely avoided "inductive" descriptions.) I use the dob (d) as a measure of characteristic distances for this purpose, and then this "criterion" becomes defined by two statements:

$$
d<x_{g} \text { (VLF regime) - The local field distributions will (usually) }
$$
be approximated by an equivalent static description. Note that this also means $\delta<\chi_{g}$, which is compatible with $c$. above (in alluvium).

$d>\lambda_{\mathrm{g}}$ (MF regime) - This category is dominated by a radiation field description, and it is also compatible for our purposes, to use plane-wave approximations. (Alluvium is essentially excluded here, of course.) In granite, since $\gamma \sim d$, it follows that $\gamma>x_{g}$, which leads to some coincident simplifications later on. 
d. Information Bandwidth. The precision with which the cavity interaction signal would be measured and interpreted will depend in part on system bandwidth. One basic criterion is the discernible increment of cavity radius:

$$
\frac{\Delta R}{R}=\frac{\dot{R}}{R} \Delta t=\alpha_{c} \Delta t \approx \frac{\alpha_{c}}{2 \Delta f}
$$

For instance if we set $\frac{\Delta R}{R}=.1$ (a $10 \%$ measurement) and $\alpha_{c} \simeq 500$ (a typical number), then $\Delta f \simeq 2.5 \mathrm{kHz}$. In the M.F. domain, this is evidently no problem, but in the VLF regime, we will see that this number is comparable to the generator or carrier center frequency. Such high-percentage modulation schemes are very difficult to instrument, implying a preference for frequencies above $\sim 10^{4} \mathrm{hz}$.

c. Cavity "Resonance" $\left(R \sim x_{g}\right)$. For underground wavelengths of the same order of dimension as the cavity, one might expect to find recognizable coherent scattering effects. The solutions of Maxwell's equations for the case of an infinitely conducting sphere imbedded in a dielectric, do exhibit a series of anharmonic mode "resonances" in the exterior field distribution. The lowest of these is characterized by a propagation constant of value:

$$
k=-(1 \pm j \sqrt{3}) \frac{c t}{2 \sqrt{k g}^{R}}
$$

This describes an oscillator whose resonant frequency is:

$$
f=\frac{\sqrt{3} c}{4 \pi \sqrt{k_{g}} R}
$$

and which has a decrement measured by the time constant:

$$
\tau=\frac{2 \pi \sqrt{\mathrm{Kg}_{\mathrm{g}}} \mathrm{R}}{\mathrm{c}} \simeq \frac{\sqrt{3}}{2} \frac{1}{\mathrm{f}}
$$


Note that this is a fraction of a cycle. This oscillator radiates away most of $i$ ts energy within one cycle. (Ref. Stratton, E.M. Theory, Sec 9.23.). In a plane-wave interaction, one might expect to generally see this as a strong effective absorption (since the re-radiation reduces the effective wave energy density) with perhaps spatially singular constructive interference effects, and noticeable phase-frequency anomalies.

For our nominal cavity example ( $5 \mathrm{kt}, \mathrm{d}=200 \mathrm{~m}$, and $\mathrm{R}^{\prime} \simeq 30 \mathrm{~m}$ ), this "asymptotic" resonance occurs at $\sim 800 \mathrm{kHz}$ for the final cavity size. In alluvium, the EM $\mathrm{mfp}$ is $\sim 12 \mathrm{~m}$ and there is no prospect of seeing it. In granite, $\gamma \sim 160 \mathrm{~m}$ and the cavity resonance should be visible. Evidently for higher yields, the resonance frequency is lower, and for earlier times, it is higher. Somewhat arbitrarily, I have assigned the range of $300 \mathrm{kHz}$ (corresponding to $\mathrm{W} \sim 100 \mathrm{kt}$, with $x_{\mathrm{g}}=50 \mathrm{~m}$ ) to $3 \mathrm{mhz}$ (corresponding to $R \sim 7 \mathrm{~m}$ with $x_{\mathrm{g}} \sim 5 \mathrm{~m}$ ) to this MF class (3). Evidently too, this class also falls in the category of "Plane-wave" approximation analysis as just defined, inasmuch as $x_{g} \lll d$.

The earth medium should not affect this model very much, since it is difficult to further damp an oscillator which is already "loaded" so heavily. As a first-order approximation, we will estimate the signal levels at a lower non-resonant frequency in the same domain (e.g., $\frac{1}{2} f_{\text {res }}$ ). The feasibility of observation of an amplitude - phase anomaly at resonance, can then be gauged by that off-resonance, "plateau", S/B number. 
Generator Energy Coupling

Evidently those frequency criteria (above) are already pointing to three broad regimes of systems possibilities:

(1) Conduction ("Earth") Currents - DC \& VLF in Alluvium

(2) (Quasistatic) Magnetic Fields - VLF in Alluvium and Granite

(3) Radiation Fields (RF "illumination") - MF in Granite

While we seek criteria which relate to optimization of the cavity field, we also are looking for guidelines for choosing tractable calculational design models. Some of these topics have relevance to Sensor design as we11.

a. The Adiabatic Equilibrium Assumption. Towards the end of cavity expansion (note figure 2 again), the logarithmic rates-of-change $\left(\frac{l}{R} \frac{\partial R}{\partial t}\right)$ become small compared to the radial frequencies $(\omega)$ of interest to us. In consequence, a reasonable calculational assumption is that the "scattered" EM fields are (instantaneously) very close to a steadystate description. In particular, their wave-trains are quasi-sinusoidal. We can then calculate the interaction on a point-by-point basis along the cavity expansion time base.

This perhaps violates the information bandwidth concept, but we remedy this to first order, by considering the instantaneous solution as a "center-frequency" representation. The bandwidth requirement would be imposed on this as an effective modulation, as indicated before. 
b. The Displacement Criterion. This arises from the relative influence of the conduction and displacement terms in Maxwell's equations: $E$ vs. $k_{g} k_{0} \frac{\partial E}{\partial t}$. For a sinusoidal field, $k_{g} k_{0} \frac{\partial E}{\partial t} \rightarrow \omega K_{g} k_{0} E$, and we write:

$$
\begin{aligned}
& \text { If } \frac{{ }^{K} g{ }{ }^{\omega}}{\sigma} \ll 1 \quad \text { conduction coupling is indicated } \\
& \text { If } \frac{{ }^{{ }_{\mathrm{g}}{ }{ } \omega}{ }^{\omega}}{\sigma .} \gg 1 \quad \begin{array}{l}
\text { radiation coupling is indicated } \\
\text { (high resistance media) }
\end{array}
\end{aligned}
$$

This is evidently not an "exclusive" criterion, as it says nothing about the quasi-static magnetic schemes. It is not necessarily true that, if $\frac{{ }^{K}{ }^{k}{ } \omega}{\sigma} \sim 1$, so-called "inductive coupling" is an optimum choice.

This one has relevance primarily in the "deep" cases: $>$ or $\ll$.

c. Field Curvature. The geometric order of the field distribution turns out to have important consequences on the feasibility estimates. By this we mean the range dependence of the "illumination" field - e.g., $r^{-1}$, $r^{-2}, r^{-3}$. (As much can also be said of the "scattered" field, of course, but one does not have as much control of that.) Evidently, if we place excitation energy (or power) high on the list, we may be best off with a coupling scheme having the lowest-order inverse dependence. Furthermore, multi-element generator arrays can be devised which will produce quasiplanewaves in the radiative domain, or which will produce quasi-static fields having two-dimensional curvature (rather than three-dimensional) in restricted regions. We will see examples of these. 
It may also be possible to improve S/B ratio by resorting to schemes which intrinsically impose a preferential field structure between the two ( $S$ or $B$ ) paths. In the next section, we will write quantitative, range-dependent descriptions and optimization formulae for the simpler source models.

d. The Quasi-Static (EM) Approximation. The condition $x_{g} \gg d$ has significance in the choice of analytic representation of the generator function. But it also has important instrumentation consequences: The phase delays between system elements may be considered as negligible. One hardware consequence of this is that we can distribute and connect generator (and sensor) elements as if they were "D.C." components with regard only to their intrinsic polarizations.

The extent to which this quasi-static field assumption is valid, may be seen by considering our central VLF example, for which $\omega \sim 2 \times 10^{4}$. Assuming that system elements are distributed on a dimensional scale of order $d=200 \mathrm{~m}$, we see that this corresponds to a relative phase angle of: $\phi=360 \frac{\sqrt{k g} \omega d}{2 \pi c} \simeq 2.4$ degrees. This is a measure of "neglect".

e. The Plane-Wave Approximation $\left(x_{g} \ll d\right)$. Again this has both problem-solving implications and hardware consequences. In this radiation domain limit, phase relationships must be preserved. Illumination (and observation) functions may be considered either in terms of independent elements of arbitrary phase, or else account must be kept of phase delays along the various propagation paths and between connected system components. 
"Experience" suggests that it would be difficult to realize a gain of more than $\sim 30 \mathrm{db}(\times 30)$ in local radiation patterns, by the use of phased arrays. But that is certainly worthwhile, especially if we could use this "twice" (at generator and at sensor) in a discriminative way, as between $S$ and $B$ paths. 


\section{Sensor Configurations}

There are three basic options for field measurement, as distinguished by the sensed field component:
(1) Magnetic
(2) Electric
(3) Electromagnetic

The significant sensor features are those which lead to acceptable Signal/Noise and Signal/Background ratios, both at the same time. As indicated before, these tend to be incompatible requirements. (What's good for one is bad for the other and vice versa.) of course, the first two sets of criteria have direct impact here also - for instance - in the removal of the scattering measurement from the regime of competitive real-time noise. A consequence is that these sensor criteria are addressed more to configurational specification.

The magnetic sensor class centers around the proverbial loop as the prototype. But in these general frequency domains, one can afford the increase in sensitivity obtainable from large (effective) areas and many turns, at the price of inductance and time constant. Ferrite cores may be used to keep the devices physically' small, in case one wants to drop them down instrumentation holes. In some geometries, one may want loops with low orthogonal and gradient sensitivities; this might be achieved by means of interlaced windings, for instance. We will also treat with at least one case involving a quadrupole loop. 
Electric sensors would involve measurement of the underground electric field, which may be related to $\sigma E$ and/or to $\omega K_{g}{ }{ }_{0} E$. Presently, there are ambiguities in the treatment of the response of "ground probes" in alluvium, and I have omitted their final evaluation. It is immaterial to the general conclusions for that medium.

An "EM" sensor involves elements which are designed to couple to both components of a radiative field - e.g., "antennas". In the studies to date, I have not encountered situations in which such structures would exhibit significant advantages over the use of the more conventional sensors in (phased) arrays. However, such sensing techniques might find application in the granite-MF systems.

a. Resonance. The trenchant feature in respect to $S / N$ is of course, the use of resonance circuits throughout the system. We have seen that the principal accommodation is to the required bandwidth for information retrieval. And also, the higher frequencies are advantageous in inverse proportion to $\frac{\Delta f}{f}$, in an arbitrarily "white" noise spectrum situation.

The higher frequencies have an instrumentation advantage in several other respects. For sensors which respond to rate (such as loops) a dominant amplitude term will be of form $\dot{B}=\omega B$. Also, since $\Delta f$ is considered invariant in the design exercise, and since $Q \simeq \frac{f}{\Delta f}$, then higher frequencies permit us to work with higher $Q$ systems. In particular, sensors operated at resonance display an implicit gain of order Q, over similar non-resonant sensors. 
In contrast, these features do not directly affect the S/B ratio; frequency selectivity does not in itself, furnish a simple discriminant between the two fields (or signal paths). However, in a sophisticated syștem, one might resort to strictly sideband demodulations, suppressing both the $S$ and $B$ carrier contributions.

b. Phase (or "Polarity"). Closely associated with resonance, is the relative phase of signal and background components. As indicated before, in the VLF domain, this amounts to keeping track of "polarity" (e.g., \pm ); in the MF domain, we have to keep account of "phase rotation" as measured by $\frac{\omega L}{v_{g}}$, for instance.

One can choose sensor and system geometries, circuits, and adjustments which involve self-cancellation or "nulling", of the background contribution, with only partial degradation of the scattered signal. The extent to which this is practically realizable, will depend on many system stability factors - some of which may not be experimentally controllable. Certain types of sensors involve a high degree of phase cancellation in their intrinsic design, such as the quadrupole loop (which amounts to two conventional loops, back-to-back), and low-gradient response loops (i.e., - conversely, loops having low quadrupole moments). c. Polarization (or Orientation). This feature provides us with the major independent "handle" on S/B ratio. Its effectiveness depends on the degree of difference in field topography and in viewing aspect of the source elements and of the conducting cavity. The field distributions adopted here as descriptive of source output, cavity re-radiation, and 
sensor response, exhibit generally weak angular dependence (e.g., sin $\theta$ ). If the relative vieving angles are sufficiently different, the transmitter and receiver elements can be oriented so that they are in each other's minima, without serious sacrifice of cavity field coupling. A self-evident criterion is that all three system elements cannot lie in one another's minima.

Perhaps the most illustrative scheme in this respect is the "crossed 10op" geometry (note figure 4) which is particularly applicable to the VLF domain. In the radiative domain, one may be able to utilize the node structure of multi-element arrays to the same end.

d. Location. Without reference to those more delicate features of Phase and Polarization, a primitive argument can be made with respect to two asymptotic extremes in location:

"Near" - (i.e., as near as possible, like "surface zero"). This usually optimizes "Signal" - but requires extreme expedients to achieve acceptable S/B.

"Far" - (i.e., far enough so that S/B becomes asymptotic). Without "ingenious devices", this usually achieves the best S/:B ratio but the Signal takes a real beating.

Intuitively, these suggest that intermediate situations may provide intermediate $S / N$ and $S / B$ conditions which may be amenable to practical improvement. In the VLF case, the Phase and Polarization criteria suggest that such a location may, in fact, optimize the signal coupling in terms of field orthogonality - as we will further develop below. (In a manner of speaking, we're right back where we empirically started.) 
e. Symmetry. This is frequently implicit in the design of most R.F. systems, because the orders of symmetry of simple elements such as loops, dipole antennas, etc., are universally understood in the R.F. community. Here I emphasize the point, because Test site conditions are not conducive to similarly intrinsic physical symmetries. One has to purposefully work at it.

One of the consequences of invoking a broad configuration symmetry requirement, might be to split the prompt diagnostic cabling system, so that each half lies on the same diameter through the array, to two identical trailer installations equidistant from S.Z. One's imagination can simply go wild on this sort of thing. 


\section{ANALYSIS DESCRIPTIONS}

This is a collection of analytic statements which find use in describing the operation and contributions of each system component along the two relevant paths. It was useful to establish at an early stage, a uniform and correspondent basis among features of common description. We will see that the various prototype systems consist, for the most part, of different combinations of such similar basic components. Numerical examples are provided here for orientation.

There is a correspondence between types' of systems, media, and frequency domain, which is reflected in the categories discussed here: Conduction Current Techniques - DC and VLF in Alluvium only. Quasi-static Magnetic Fields - VLF in Alluvium and Granite. VLF Hardware Aspects - Trasmitter; Receiver. Radiative EM Field Techniques - MF and HF in Granite only.

The class of systems using Electric field generators has been arbitrarily excluded as a technique. The achievable generator field strengths (e.g., in Granite) lead to a cavity field energy density far below that attainable by other schemes. 
Some Dimensional Relations in the Media:

$$
\begin{array}{lll} 
& \text { Alluvium }\left(\frac{1}{\sigma}=400\right) & \text { Granite }\left(\frac{1}{\sigma}=10^{4}\right) \\
& \kappa_{g}=9 \text { for both media } & v_{g}=\frac{c}{\sqrt{\kappa_{g}}}=10^{8} \\
\omega & 2 \times 10^{4} \mathrm{Av} & 2 \times 10^{6} \mathrm{Av} \\
v & \sim 3 \mathrm{kHz} & \sim 300 \mathrm{kHz} \\
x & 10^{5} \mathrm{~m} & 10^{3} \mathrm{~m} \\
x_{g}=\frac{v_{g}}{\omega} & 5 \times 10^{3} \mathrm{~m} & 50 \mathrm{~m} \\
& \delta=180 \mathrm{~m} & \gamma=160 \mathrm{~m}
\end{array}
$$

The Prototype Test $(W=5 \mathrm{kt})$

$$
\begin{array}{ll}
R_{f}=16.5 \mathrm{~W}^{1 / 3} & \text { meters } \rightarrow 28 \mathrm{~m} \\
R_{C}=50 \mathrm{~W}^{1 / 3} & \text { meters } \rightarrow 86 \mathrm{~m} \\
d=115 \mathrm{~W}^{1 / 3} & \text { meters } \rightarrow 200 \mathrm{~m}
\end{array}
$$

This choice of numbers avoids estimation difficulties when $x_{g} \sim \delta$ or $x_{g} \sim \gamma$, and $x_{g} \sim d$.

Note

$$
\frac{\delta}{x_{g}} \sim \frac{1}{25} \quad \frac{x_{g}}{\lambda} \sim \frac{1}{20}
$$




\section{Power-Voltage Considerations}

In designing an "illuminator", two pragmatic factors dominate the hardware: The power requirement, and the maximum voltages. Evidently one could endlessly pyramid the power, in efforts to get better signals. Aside from interference/damage considerations (to T\&F and diagnostic circuits for example), there is basically an economic limit to this, which can be best illustrated by comparing some power system figures, relative to typical event costs:

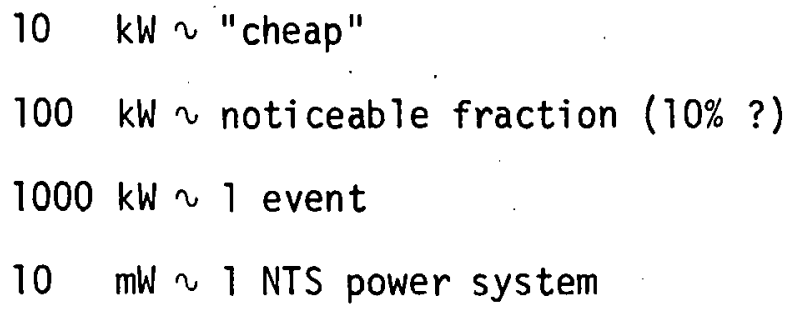

For evaluation purposes, the power ceiling is taken here as $100 \mathrm{~kW}$.

The voltage limit arises from insulation, corona, etc., considerations. Here again there are practical problems in large test site configurations, especially where the system must be designed to withstand large mechanical forces as we 11:

$3 \quad k V$ is easy

$10 \mathrm{kV}$ is modestly difficult

$30 \mathrm{kV}$ is hard work

$100 \mathrm{kV}$ is a major undertaking

For evaluation purposes, this is taken as $10 \mathrm{kV}$. 
Conduction Current Schemes

Topics included:

The Laplace Correspondence.

Earth Current Generators. Real Electrode Configurations.

Field and Current Distributions.

The Immersed Spherical Conductor; moment and fields. 


\section{The Lap lace Correspondence}

This helpful concept permits us to exchange known descriptions among similar quasi-static problems. Electric and Current fields which satisfy the potential definition $\nabla^{2} V \equiv 0$ between conductors described by similar boundary conditions, have identical field and stream lines. Analytical descriptions are directly comparable, if we take:

$$
J=\sigma E . \quad i=\frac{\sigma}{\kappa_{g} K_{0}} q \quad R=\frac{\kappa_{g} K_{0}}{\sigma C}
$$

(and so forth)

Earth Current Generators

As the primitive earth contact,

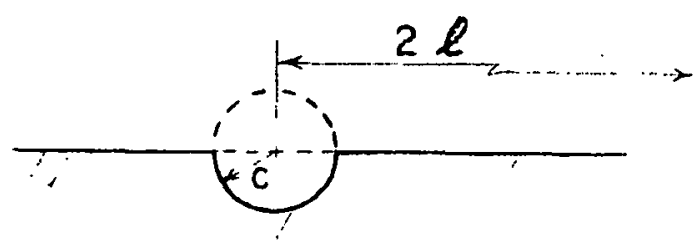
we use a half cylinder of radius $c$ (of length $L$ transverse to paper). There is another similar, parallel conductor at a distance $2 \ell$; we wish to determine the resistance between them.

Consider first, the capacitance (per unit length) between two buried cylinders:

$$
c_{l}=\frac{\pi k^{k} 0}{\cosh ^{-1}(2 \ell / 2 c)}
$$

By the $\nabla^{2}$ principle: $\quad R_{\ell}^{\prime}=\frac{\cosh ^{-1}(\ell / c)}{\pi \sigma}$

Since the geometry is bisected by the earth's surface, the actual resistance will be twice as great, and also, neglecting end effects, $R=\frac{1}{L} R_{\ell}^{\prime}$, whence:

$$
R=\frac{2 \cosh ^{-1}(\ell / c)}{\pi \sigma L}
$$


Consider some practical cases: $L=\ell \sim 200 \mathrm{~m} \cdot \frac{1}{\sigma} \sim 400$. Test a range of $c$ from $\frac{1}{2} \mathrm{~m}$ to $5 \mathrm{~m}$.

$$
\begin{array}{lccccc} 
& & R=\frac{4}{\pi} \cosh ^{-1}\left(\frac{200}{c}\right) & \sim 1.27 \cosh ^{-1}\left(\frac{200}{c}\right) & \\
C= & .5 & 1 & 2 & 5 & \mathrm{~m} \\
\mathrm{R}=8.5 & 7.6 & 6.7 & 5.6 & \Omega
\end{array}
$$

We see that the problem is insensitive to contact radius. Economics will dictate the smaller numbers here and one would be inclined to specify $c \sim 2 \mathrm{ft}$ here so that $R \sim 8 \Omega$. This is the value we will use in the evaluation problems.

Real Electrode Configurations. The pragmatics enter again here. It would probably be expensive to install "good" contacts of that type over uneven terrain. So we will segment the conductor $n$-fold and also install each segment vertically, such that $L \simeq n h$, where $h$ is the conductor depth. Preferably, nh $>$ L. Thus, if $20 \mathrm{~m}$ is a practical depth, $n \sim \frac{\mathrm{L}}{\mathrm{h}} \sim \underline{10}$ (at least).

We indicated $2 \mathrm{ft}$. diam. conductors. We can probably get by with less, say $1 \mathrm{ft}$. pipes, if we also adopt a diligent earth conditioning program - e.g., we modify the conductivity locally, by $\mathrm{CuSO}_{4}$ (or $\mathrm{NaC} \ell$ ) impregnation. This might be done by perforating the conductor pipes and periodically filling them with solution.

Net (and individual) resistance monitoring is certainly in order. 
$-53-$

Derivation of Field and Current Distributions

Due to Parallel Probe System (First-order)

We go back to the half-cylinder idealization. This is a two-dimensional problem; it uses the Laplace Correspondence Principle.

We have, for the potential relative to the central plane:

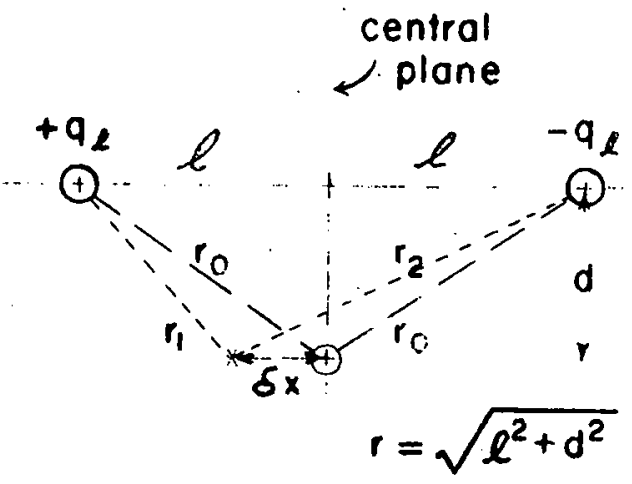

$$
V=\frac{q_{l}}{2 \pi \kappa_{g^{k}}} \ln \frac{r_{2}}{r_{1}}
$$

Where $( \pm) q_{l}$ is the (line) charge on conductors normal to the paper, as

and:

$$
E_{X}=\frac{\partial V}{\partial x}
$$
Indicated.

Consider a (small) displacement $\delta_{X}$ :

$$
\begin{gathered}
r_{1}=r_{0}-\delta r=\sqrt{(\ell-\delta x)^{2}+d^{2}} \\
r_{1}=r_{0}+\delta r=\sqrt{(\ell+\delta x)^{2}+d^{2}} \\
r_{1} \simeq \sqrt{\ell^{2}+d^{2}-2 \ell \delta x}=\left(1-\frac{l \delta x}{\ell^{2}+d^{2}}\right) \sqrt{\ell^{2}+d^{2}}=r_{0}-\frac{l \delta x}{\sqrt{\ell^{2}+d^{2}}} \\
r_{2} \simeq \sqrt{\ell^{2}+d^{2}+2 \ell \delta x}=\left(1+\frac{\ell \delta x}{\ell^{2}+d^{2}}\right) \sqrt{\ell^{2}+d^{2}}=r_{0}+\frac{l \delta x}{\sqrt{\ell^{2}+d^{2}}}
\end{gathered}
$$

Hence $\quad \delta r=\frac{\ell}{r_{0}} \delta x$

$$
\text { or } \delta x=\frac{r_{0}}{l} \delta r
$$


The corresponding potential increment is:

$$
\begin{gathered}
\delta V=\frac{q_{l}}{2 \pi \kappa_{0}} \ln \frac{r_{0}+\delta_{r}}{r_{0}-\delta}=\frac{q_{l}}{2 \pi \kappa_{0}}\left\{\ln r_{0}\left(1+\frac{\delta}{r_{0}}\right)-\ln r_{0}\left(1-\frac{\delta}{r_{0}}\right)\right\} \\
=\frac{q_{l}}{2 \pi \kappa_{0}}\left\{\ln \left(1+\frac{\delta}{r_{0}}\right)-\ln \left(1-\frac{\delta}{r_{0}}\right)\right\} \simeq \frac{q_{l}}{\pi \kappa_{0}} \frac{\delta_{r}}{r_{0}} \\
\left|\frac{\partial V}{\partial X}\right|=\frac{\delta V}{\delta X}=E X=\frac{q_{l}}{4 \pi \kappa_{g} k_{0}} \frac{l}{r_{0}^{2}} .
\end{gathered}
$$

Then

The Laplace correspondence leads to: $q_{\ell}=\frac{K g^{K} \varrho}{\sigma} i_{\ell}$

So that: $\quad E=\frac{i l}{\pi \sigma}\left(\frac{l}{r_{0}^{2}}\right)$ and $J=\frac{i l}{\pi}\left(\frac{l}{r_{0}^{2}}\right)$

where $i_{\ell}$ is the current injected per unit length of conductor. In our problem, the geometry is bisected by the real horizontal ground plane, so that the current density is twice as great for a given line current. Also, neglecting end effects, $i_{\ell}=\frac{I}{L}$ where $I$ is the total current.

Then

$$
J=\frac{2 I}{\pi L} \frac{l}{l^{2}+d^{2}}
$$

Example $\quad l=d=L=200$

$$
\text { and } \quad I=100 \text { amps }
$$$$
\frac{1}{\sigma}=400
$$

Then at the Cavity:

$$
J_{0}=\frac{2 \times 10^{2}}{\pi \times 2 \times 10^{2}} \times \frac{200}{200^{2}+200^{2}}=\frac{1}{400 \pi} \simeq 10^{-3} \mathrm{amps} / \mathrm{m}^{2}
$$

and

$$
E_{c}=\frac{J_{C}}{\sigma}=400 \times 10^{-3}=.4 \mathrm{~V} / \mathrm{m}
$$


The Immersed Spherical Conductor

This approximates the magnetic field due to a spherical conductor (radius a) immersed in a uniform current density. Here again we invoke the Laplace correspondence. In a formerly uniform Electric field $E_{0}$, the surface charge density is:

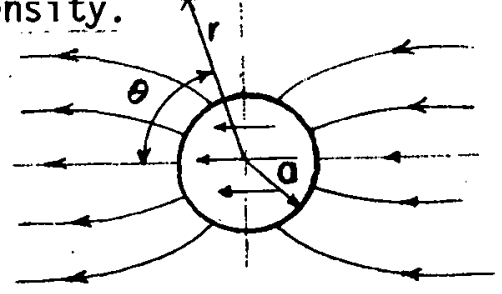

$$
q_{S}=3 k_{g{ }{ }_{0} E_{0}} \cos \theta \quad \text { (Ref: Stratton, p. 205) }
$$

If a conductive medium $\sigma$ surrounds the sphere, the field lines remain unchanged, provided that:

$$
\mathrm{J}=\frac{\sigma}{\mathrm{K}_{\mathrm{g}} \mathrm{K}_{0}} \mathrm{q}_{\mathrm{s}}=3 \sigma \mathrm{E}_{0} \cos \theta=3 \mathrm{~J}_{0} \cos \theta
$$

I define the equivalent Q.S. current moment as:

$$
p=I \delta \ell=2 \int_{0}^{\pi / 2} J a \cos \theta d s
$$

where $d s=2 \pi a^{2} d(\cos \theta)$

(The factor 2 arises from reflection around equatorial plane.)

$$
p=12 \pi a^{3} J_{0} \int_{0}^{\pi / 2} \cos ^{2} \theta d(\cos \theta) \rightarrow 4 \pi a^{3} J_{0}
$$

Using the quasistatic dipole approximation, the "side view" field magnitude is:

$$
B=\frac{\mu_{0} p}{4 \pi} \frac{\sin \theta}{r^{2}}=\mu_{0} J_{0} \frac{a^{3}}{r^{2}} \sin \theta
$$

The principal approximation assumed here, is that the current distribution at (and over) the cavity is relatively uniform prior to its growth. 
Example: We take the previous current density: $J_{0}=10^{-3}$ and assume that the sensor is "overhead": $r \sim d$. Then for the prototype explosion:

$$
B=\mu_{0} J_{0} \frac{R^{3}}{d^{2}} \simeq 4 \pi \times 10^{-7} \times 10^{-3} \times \frac{(28)^{3}}{(200)^{2}} \simeq 7 \times 10^{-10} \mathrm{Web} / \mathrm{m}^{2}
$$

which is of order one gamma (at max cavity radius)! 


\section{Quasistatic Magnetic Fields}

Topics included:

Moments Defined; The Diamagnetic Sphere

The Dipole Equations

The Exact Loop Expressions

Some of the definitions here apply as well to the radiative field descriptions. 
Moments

This is another ambiguously used term, with various suffixes and prefixes. For our purposes here, we need just two basic definitions: The current moment $p$, and the magnetic moment $m$.

The Current Moment is defined as $p=i h$, where $i$ is the current impressed on a conductor of length $h$. Evidently for a continuous harmonic oscillator $i=j \omega q$ so that $p=j \omega m_{e}$, where $m_{e}$ is the classical electric moment.

For an idealized rectangular cylinder with uniform current density J (evidently "impossible"):

$$
p=\pi a^{2} l_{0}=I_{0} l
$$

The Magnetic Moment is basically defined in terms of an infinitesimal magnetized bar. I will use the "small loop" definition: $m=\pi a^{2} \mathrm{Ni}$, where $\mathrm{a}$ is the radius of the $\mathrm{N}$-turn loop carrying the current $\mathrm{i}$.

The Diamagnetic Sphere - for a perfectly diamagnetic sphere placed in a previously uniform field $B_{0}$, the equivalent magnetic moment is:

$$
m=4 \pi a^{3} \frac{B_{0}}{\mu_{0}}
$$

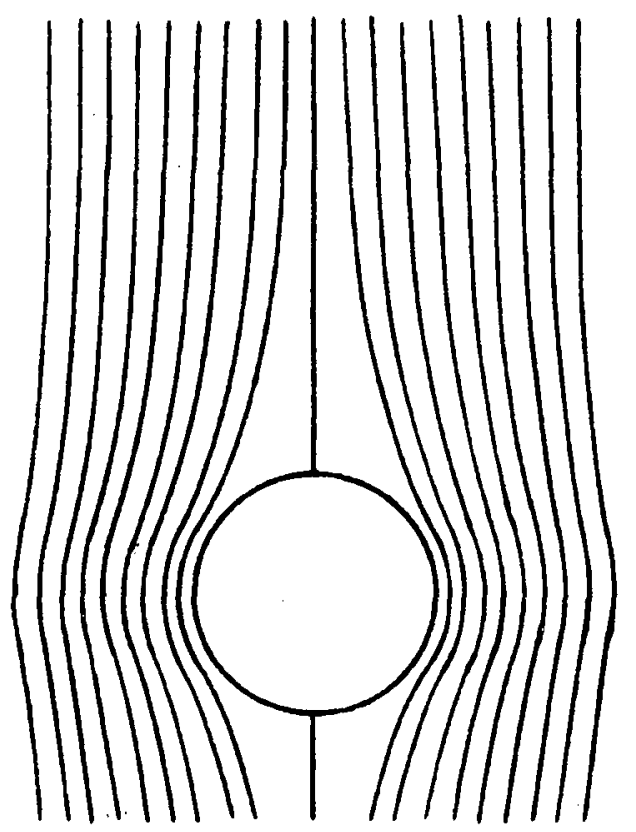


We get this by taking the "crystalline sphere" problem to its diamagnetic limit: $\mu^{\prime} \rightarrow$ o (Smythe III, p. 352). The equivalent loop current is:

$$
I^{\prime}=\frac{4 a}{\mu_{0}} B_{0}
$$

Examples: Consider a $10 \mathrm{~m}$ radius conducting sphere placed in an R.F. field of ten gamma $\left(10^{-8}\right.$ Web $\left./ \mathrm{m}^{2}\right)$ :

$$
m=4 \pi \times 10^{3} \times \frac{10^{-8}}{4 \pi \times 10^{-7}}=100 \mathrm{mks}
$$

Compare with a 1 turn 10op, $100 \mathrm{~m}$ radius and 100 amps of excitation current:

$$
m=\pi \times 10^{4} \times 100 \simeq 3 \times 10^{6} \mathrm{mks}
$$

This begins to provide an appreciation of the "visibility" problem.

\section{The Dipole Equations}

As indicated under "Criteria and Constraints", we managed to avoid (with one or two exceptions) that difficult set of problems for which $x_{g} n, R$, or $x_{g} \sim \delta$, at least in these evaluations. The "exact" program intentionally treats that frequency domain, and as also indicated before, its answers do not depart wildly from reasonable extrapolations from the neighboring domains.

As long as the source (or sensor) object is "sufficiently" smaller than $x_{g}$, a useful approximation for its first-order field pattern is a dipole - electric or magnetic, as appropriate. And further, the significant field function will associate with frequency domains according to the "strength" of the range terms $\left(\frac{\chi_{\mathrm{g}}}{r}\right)^{\mathrm{x}}$ 
Here in particular, it is important to state clear definitions. Various references are ambiguous, both in arithmetic statements, and in depiction of terms such as magnetic dipole, charge dipole, radiative dipole, dipole moments, etc.

The Complete Expressions. The references used here were Harnwel1, Stratton and Smythe. The dipole definitions used by these authors are not completely reconcilable. However, if I restrict application to small elements, to amplitude functions only, and to single, continuous, discrete frequencies, then all these forms converge to the same "infinitesimal" description:

The Dipole Moments are defined as follows:

The current dipole is $p=$ ih

The magnetic dipole is $m=i A$

where $i$ is the "excitation" current

$h$ is the dipole length

$A$ is the loop area (e.g., $A=\pi a^{2} N$ )

Note that this current dipole corresponds to the usual infinitesimal radiative dipole used in $R F$ engineering texts (e.g., Terman). It is not the same as the "electric dipole": $q h=\left|j \frac{i}{\omega} h\right|$. The magnetic dipole is also sometimes called the loop dipole. 
$-61-$

Current Dipole Fields

$$
\begin{aligned}
& E_{r}=\frac{1}{2 \pi \kappa_{0} \omega}\left(\frac{1}{r^{3}} \pm j \frac{1}{x_{r^{2}}}\right) \cos \theta p \\
& E_{\theta}=\frac{1}{4 \pi \kappa_{0} \omega}\left(\frac{1}{r^{3}} \pm j \frac{1}{x_{r^{2}}}-\frac{1}{x^{2} r}\right) \sin \theta p \\
& B_{\phi}=\frac{\mu_{0}}{4 \pi}\left(\frac{1}{r^{2}} \pm j \frac{1}{x_{r}}\right) \sin \theta p
\end{aligned}
$$

Magnetic Dipole Fields

$$
\begin{aligned}
& B_{r}=\frac{\mu_{0}}{2 \pi}\left(\frac{1}{r^{3}} \pm j \frac{1}{\frac{x^{2}}{r^{2}}}\right) \cos \theta m \\
& B_{\theta}=\frac{\mu_{0}}{4 \pi}\left(\frac{1}{r^{3}} \pm j \frac{1}{\left.\frac{x^{2}}{r^{2}}-\frac{1}{x^{2} r}\right) \sin \theta m}\right. \\
& E_{\phi}=\frac{\mu_{0} \omega}{4 \pi}\left(\frac{1}{r^{2}} \pm j \frac{1}{\pi_{r}}\right) \sin \theta m
\end{aligned}
$$

In our usage here, the phase specification of the so-called "induction" term is immaterial.

These expressions are surprisingly good approximations. In a typical case, the quasi-static on-axis field for a loop was calculated by both the "exact" expression and the dipole equations - to better than $20 \%$ agreement.

In the following limit expressions, we have:

$$
\frac{1}{4 \pi \kappa_{0} \omega} \rightarrow\left(\frac{\mu_{0}}{4 \pi}\right) x_{g} v_{g} \quad v_{g}=\omega x_{g}=\frac{c}{\sqrt{\kappa_{g}}}
$$


The Quasistatic Limit (DC and VLF)

Current Dipole $\quad E_{r}=2\left(\frac{{ }_{o} p}{4 \pi}\right)\left(x_{g} v_{g}\right)\left(\frac{\cos \theta}{r^{3}}\right)$

$$
\begin{aligned}
& F_{\theta}=\left(\frac{\mu_{0} p}{4 \pi}\right)\left(x_{g} v_{g}\right)\left(\frac{\sin \theta}{r^{3}}\right) \simeq\left(\frac{x_{g}}{r}\right) v_{g} B_{\phi} \\
& B_{\phi}=\left(\frac{\mu_{0} p}{4 \pi}\right)\left(\frac{\sin \theta}{r^{2}}\right)
\end{aligned}
$$

$\underline{\text { Magnetic Dipole }} \cdot B_{r}=2\left(\frac{{ }^{\mu}{ }^{m}}{4 \pi}\right)\left(\frac{\cos \theta}{r^{3}}\right)$

$$
\begin{aligned}
& B_{\theta}=\left(\frac{\mu_{0} m}{4 \pi}\right)\left(\frac{\sin \theta}{r^{3}}\right) \\
& E_{\phi}=\left(\frac{\mu_{0} m}{4 \pi}\right)\left(\frac{v_{g}}{x_{g}}\right)\left(\frac{\sin \theta}{r^{2}}\right) \simeq\left(\frac{r}{x_{g}}\right) v_{g} B_{\theta} \quad\left(r \ll x_{g}\right)
\end{aligned}
$$

Field Vector Direction. Note the angle $\psi$ defined by:

$$
\tan \psi=\frac{E_{\theta}}{E_{r}}=\frac{B_{\theta}}{B_{r}}=\frac{1}{2} \tan \theta
$$

The direction of the field lines (at $r, \theta$ ) relative to the dipole axis, is given by $(\theta+\psi)$.

The Exact Expressions

For Magnetic Fields Due to a Loop (Quasi-Static case)

(Ref. Stratton, p. 263/Smythe, I-266, III-291)

The general expressions, for a loop of radius a, are:

$$
\begin{aligned}
& B_{r}=\frac{\mu_{0}}{2 \pi} N I \frac{z}{r \sqrt{(a+r)^{2}+z^{2}}}\left[-K+\frac{a^{2}+r^{2}+z^{2}}{(a-r)^{2}+z^{2}} E\right] \\
& B_{z}=\frac{\mu_{0}}{2 \pi} N I \frac{1}{\sqrt{(a+r)^{2}+z^{2}}}\left[K+\frac{a^{2}-r^{2}-z^{2}}{(a-r)^{2}+z^{2}} E\right]
\end{aligned}
$$


The particular cases of interest to us are:
(1) The equatorial plane $z=0$
(2) The central axis
Cylindrical coord.

Note that $K(k), E(k)$ where $k^{2}=\frac{4 r}{(a+r)^{2}}=\frac{4 x}{(1+x)^{2}}$

and $\quad k=\frac{2 \sqrt{x}}{1+x} \quad x=a / r$

$K$ and $E$ are elliptic integrals of first and second kind, respectively.

They are tabulated according to $\sin ^{-1} k$. Some "end" values:

$$
\begin{array}{rlrl}
\sin ^{-1} k & =0^{\circ} & 90^{\circ} . \\
K & =\pi / 2 & \infty \\
E & =\pi / 2 & 1.000
\end{array}
$$

Equatorial Plane $Z=0 \quad B_{r}=0$

$$
\begin{aligned}
B_{z} & =\frac{\mu_{0} N I}{2 \pi} \frac{1}{(a+r)}\left[K+\frac{(a-r)(a+r)}{(a-r)^{2}} E\right] \\
& =\frac{\mu_{0} N I}{2 \pi}\left[\frac{K}{a+r}+\frac{E}{a-r}\right]=\frac{\mu_{0} N I}{2 \pi r}\left\{\frac{K}{1+x}-\frac{E}{1-x}\right\}
\end{aligned}
$$

Central Axis $\quad r=0 \quad x=0 \quad k=0 \quad \sin ^{-1} k=0$

Note $B_{r} \rightarrow \frac{0}{0}$; Symmetry requires $B_{r} \equiv 0$

$$
\begin{aligned}
B_{z} & =\frac{\mu_{0} N I}{2 \pi} \frac{1}{\sqrt{a^{2}+z^{2}}}\left[K+\frac{a^{2}-z^{2}}{a^{2}-z^{2}} E\right] \quad K, E=\pi / 2 \\
& =\frac{1}{2} \mu_{0} N I \frac{a^{2}}{\left(a^{2}+z^{2}\right)^{3 / 2}}
\end{aligned}
$$

In our language: $B=\frac{1}{2} \mu_{0} N I \frac{a^{2}}{\left(a^{2}+r^{2}\right)^{3 / 2}}$ 
Example: Equatorial Plane

In our range of interest, $x=a / r \sim .1$ (for "side illumination")

$$
\text { So } \begin{array}{rlrl}
k & =\frac{2 \sqrt{.1}}{1.1}=\frac{.63}{1.1}=.57 & \sin ^{-1} k & =35^{\circ} \\
\text { and } k & =1.731 & E & =1.432
\end{array}
$$

$$
\left|\left\{\frac{K}{1+x}-\frac{K}{1-x}\right\}\right|=\left|\frac{1.731}{1.7}-\frac{1.432}{.9}\right|=.020
$$

Then: $\quad B_{z}=\frac{1}{100} \frac{\mu_{0} N I}{\pi r}$

Compare the magnetic dipole approximation:

$$
B=\frac{\mu_{0} m}{4 \pi} \frac{1}{r^{3}} \rightarrow \frac{\mu_{0} \pi a^{2} N I}{4 \pi r^{3}} \rightarrow\left(\frac{a}{r}\right)^{2} \frac{\mu_{0} N I}{4 r}
$$

For our example

$$
B=\frac{1}{100} \frac{\mu_{0} N I}{4 r}
$$

which is different by the factor $\pi / 4 \sim .8$. 


\section{VLF Hardware Aspects}

Topics included here:

Transmitter Generator Loop Design

Resonance and $Q$

Power Req't - Earth-Coupled Case; $4 \pi$ Criterion

The Optimum Illumination Problem

Receiver Sensor Loop Design; Resonance Criterion

Loop Signals; Fringe Contributions

Earth Probe Signals

Bandwidth Criteria

The Optimum (Dipole) Coupling Problem

Again some of this also applies to the radiative domain and methods. Generator considerations were restricted here to Loop configurations; mainly because there seems to be no other element geometry which couples energy efficiently for system dimensions small compared to wavelength. (The earth current technique as applied to the VLF domain is discussed under specific cases.)

Certain special sensor schemes (such as a quadrupole 10op) will likewise be developed in connection with the specific case discussions. 


\section{Transmitter Elements}

\section{Generator Loop Design}

Here we consider L.F. loops of small numbers of turns $N$, with very large radii a $\left(10 \mathrm{~m}\right.$ to $\left.10^{3} \mathrm{~m}\right)$, and with "wire" ratios (really cables or bars) $\left(\frac{a}{w}\right)$ in the range $10^{2}$ to $10^{4}$.

Basic Parameters. Even though the conductor dimensions are quite different, much of what is said for sensor loops, applies here too.

$$
L=\mu_{0} N^{2} a(\ln (a / w)-7 / 4)=\mu_{0} N^{2} a f_{S}
$$

$f_{s}$ again lies in the same range (as for sensor loops), so that I use a central value for estimation purposes: $\bar{f}_{s}=6$.

$$
\begin{gathered}
L=\mu_{0} N^{2} a f_{s} \quad R=\frac{2 N \rho a F_{h}}{w^{2}}=\frac{2 \pi N \rho a F_{h}}{A} . \\
Q=\frac{\omega L}{R}=\frac{\frac{2}{2} \mu_{0} \omega N f_{s} w^{2}}{\rho F_{h}}=\frac{1}{2 \pi} \mu_{0} \omega N A \frac{f_{s}}{F_{h}} \frac{1}{\rho}
\end{gathered}
$$

(Note that ${ }_{2}^{3} \mu_{0} \mathrm{Nf}_{S} \mathrm{~W}^{2}$ has the appearance of a "specific inductance".)

$F_{h}$ is the "hollow tube" factor (of order 10). It represents the "hollowness" of practical large conductors. 
$-67-$

Self-Capacitance - Large transmitter loops. These loops have a small number of turns and a different approach is used. The conductor is modeled as a cylindrical capacitance with different effective fields around it. Note also that we assume air dielectric - in all probability, the construction would require non-circuital (but metallic) strength members in an open geometry.

$$
C_{L}=\frac{2 \pi \kappa_{0}}{\ln \frac{w+f_{v} w}{w}}=\frac{2 \pi \kappa_{0}}{\ln \left(1+f_{v}\right)} \quad \text { (per meter) }
$$$$
C_{T}=2 \pi a c_{L}=\frac{4 \pi^{2} k_{o} a}{\ln \left(1+f_{v}\right)}
$$

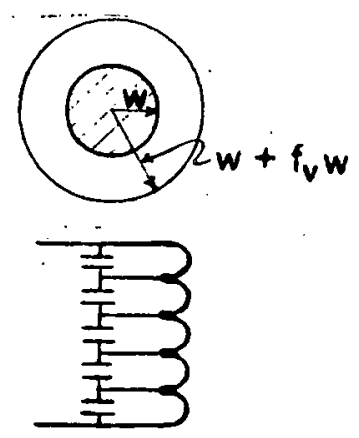

Most of the effective capacitance will be to neighboring conductors of highest relative (instantaneous) potential, both \pm . In the voltage/ capacitive division model, we account for this by assuming that the effective number of capacitances is just half of the per turn number, so that:

and

$$
1 / C \simeq \frac{N}{{ }_{i_{2}} C_{T}}
$$

This model is dependent on $\mathrm{N}$. 
An "Open-Coil" Resonance Criterion. Self-resonance occurs at $\omega=\frac{1}{\sqrt{\mathrm{LC}}}$

$$
L C=\left(\mu_{0} N^{2} a f_{s}\right)\left(\frac{2 \pi^{2} \kappa_{o} a}{N \ln (1+f)}\right)=\frac{2 \pi^{2} N a^{2}}{C_{0}^{2}} \frac{f_{s}}{\ln \left(1+f_{v}\right)}
$$

and

$$
w=\frac{c_{0}}{\sqrt{N} \pi a} \sqrt{\frac{\ln \left(1+f_{v}\right)}{2 f_{s}}}
$$

If we apply the "tuning" criterion - i.e., $\omega \sim h \omega_{0}-$ and set $L C=\frac{1}{h^{2} \omega_{0}^{2}}$, obtain:

$$
\begin{aligned}
& N^{2}=\frac{x_{0}^{2}}{\pi^{2} h^{2}\left(\frac{2 f_{s}}{\ln \left(1+f_{v}\right)}\right)} \\
& \sqrt{N a}=\frac{x_{0}}{\pi h \sqrt{\frac{2 f_{s}}{\ln \left(1+f_{v}\right)}}}
\end{aligned}
$$

Note that in a practical average case, $\frac{2 f_{s}}{\ln \left(1+f_{v}\right)} \sim \frac{2 \times 6}{.4} \sim \underline{30}$.

Thus: $\sqrt{\mathrm{N} a}=\frac{x_{0}}{17 \mathrm{~h}} \quad$ (Loose-winding criterion). 
Example: (Transmitter Loop) $\frac{\mathrm{a}}{\mathrm{w}}=10^{3} \quad \mathrm{a}=100 \mathrm{~m}, \mathrm{w}=.1 \mathrm{~m}$

$$
\begin{aligned}
& F_{h} \sim 10 \quad N=5 \quad f_{v} \simeq .5 \\
& C=\frac{2 \pi^{2} \times 10^{-9} \times 10^{2}}{36 \pi \times 5 \times .4} \sim 10^{-8} \mathrm{fds} \quad(\text { which is "believable") } \\
& L=4 \pi \times 10^{-7} \times 25 \times 100 \times 6=.02 \text { hen } \\
& R=\frac{2 \times 5 \times 1.7 \times 10^{-8} \times 100 \times 10}{.07}=.017 \Omega \\
& \omega=\frac{C}{\sqrt{N} \pi a} \sqrt{\frac{\ln \left(1+f_{v}\right)}{2 f}}=\frac{3 \times 10^{8}}{\sqrt{5} \times 10^{2}} \sqrt{\frac{.4}{12}} \simeq \frac{8 \times 10^{4}}{\text { (self-resonance) }} \\
& Q=\frac{\omega L}{R}=\frac{.16 \times 10^{4}}{.017} \sim 10^{5}(!)
\end{aligned}
$$

(which suggests that radiation and environment coupling losses will dominate the $Q$ of such systems.)

Resonance and $Q$

Among the critical factors which constrain resonant R.F. system component specification, are the required transmitter power and the receiver bandwidth. In resonant systems, both of these can be closely related to "Q", which is of course, the measure of the ratio of stored energy to energy loss per cycle: The stored energy is often represented as appearing as a "circulating" current (I) between the reactive components. 
The Series Definition - Consider a circuit segment which can be represented in terms of lumped reactive elements $L$ and $C$, and a series resistance $R$. Then the circuit $Q$ is defined as:

$$
Q=\frac{Z}{R}=\frac{\omega L}{R}=\frac{1}{\omega C R}
$$

Decrement - This resonance then also has a decrement characterized by $Q$; for $Q \gg>1$, it is $\delta \sim \frac{\pi}{Q}$, such that $e^{-\delta}$ measures the fractional decrease in amplitude of successive cyclic maxima (of same polarity). The amplitude will thus fall by $e^{-1}$ in $\frac{Q}{\pi}$ cycles, requiring a time $\tau=\frac{2 Q}{\omega}=\frac{Q}{\pi f}$.

Power Loss - Consider the stored energy $\varepsilon$, and the rate of power dissipation P:

$$
\begin{aligned}
& \varepsilon=I_{2} L_{L} I^{2}=I_{2} C V^{2}=\frac{1}{2 \omega} \frac{V^{2}}{Z}=\frac{1}{2 \omega} \cdot I^{2} Z \\
& P=I^{2} R=\left(\frac{V}{Z}\right)^{2} R=\frac{V^{2}}{Q Z}=i V
\end{aligned}
$$

where $i=\frac{V}{Q Z}$ is the excitation energy required from outside the circuit segment (at voltage $V$ ), in order to maintain the defined operating level. Evidently also: $\quad I=Q i$

This power loss, as represented by $R$, may include both ohmic (internal and environmental), and radiative contributions. Evidently we will have to be careful how we characterize R - i.e., how we "add up" the contributions. 
Parallel Loading - Q can equally well be defined in terms of a parallel equivalent resistance $R^{\prime}$, "across" the reactive components. In that case, $i=\frac{V}{R^{\top}}$ - the excitation current goes directly into resistive heat, and the lumped reactance can be pictured as an infinite impedance "floating" across the power source. We write instead:

$$
P=\frac{V^{2}}{R^{1}}=\left(\frac{V}{Z}\right)^{2} R=\frac{V^{2}}{Q Z}=i V
$$

The correspondence is thus defined by: $R R^{\prime}=Z^{2}$ and it follows that $Q=\frac{R^{\prime}}{Z}$

and so forth.

Power Req't - Earth-Coupled Case (Alluvium).

There are several "first-order" ways to "do" this problem.

First. Consider an alluvial earth as a coupled, one-turn, resistive load:

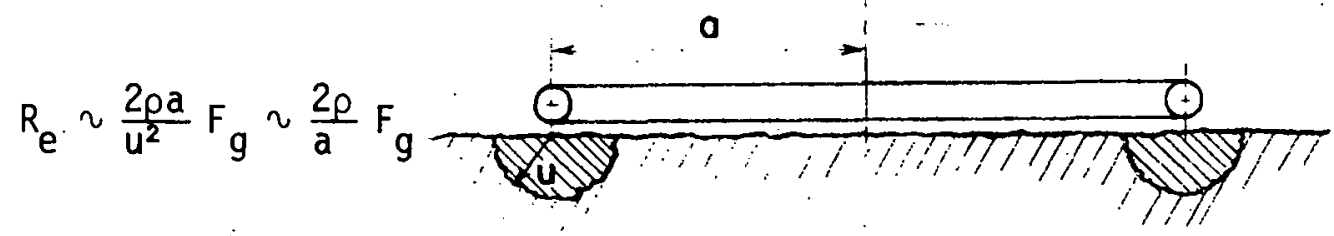

$F_{g}$ is a geometrical "fudge factor", of order 2-3.

Second. Consider the current induced in the earth by the B field.

$$
\begin{gathered}
\oint \dot{B} d s=A \dot{B}=\int E d l=\frac{1}{\sigma} \int J d l=E L \\
\pi a^{2} \omega B \sim E 2 \pi a \\
E=\frac{\pi a^{2} \omega B}{2 \pi a}=\frac{1}{2} \omega B a
\end{gathered}
$$


Power loss per unit volume is $\sigma E^{2}$

and

$$
P=\sqrt[3]{2} \omega^{2} B^{2} a^{2} \sigma
$$

Take Q.S. B $\frac{1}{2} \mu_{0} N I \frac{1}{a}$

So

$$
P \simeq \frac{1}{4} \omega^{2}\left(\frac{3}{4} \mu_{0}^{2} N^{2} I^{2} \frac{1}{a^{2}}\right) a^{2} \sigma=\frac{1}{16} \mu_{0}^{2} \sigma \omega^{2} N^{2} I^{2}
$$

We take $V \sim 8 a^{3}$

$$
P=P V=\frac{1}{2} \mu_{0}^{2} \sigma \omega^{2} N^{2} a^{3} I^{2}
$$

Then

$$
P=\left(\frac{\mu_{0} \sigma \omega}{2}\right)^{2} \frac{2}{\sigma} N^{2} a^{3} I^{2}=N^{2} I^{2} R_{e}
$$

also

$$
\begin{array}{ll}
\delta=\sqrt{\frac{2}{\mu_{0} \sigma \omega}} \text { so that: } \quad\left(\frac{1}{\sigma}=\rho\right) \\
\mathrm{R}_{\mathrm{e}} \approx \frac{2 \rho \mathrm{a}^{3}}{\delta^{4}} \mathrm{~F}_{\mathrm{g}}
\end{array}
$$

In particular if $\delta \sim a$, then the two expressions converge to:

$$
\mathrm{R}_{\mathrm{e}} \sim \frac{2 \rho}{\mathrm{a}} \mathrm{F}_{\mathrm{g}}
$$

which is as close as we can come by these rough methods. It is reasonable when all dimensions are similar.

Example: $\quad \rho \sim 400 \Omega \mathrm{m} \quad \mathrm{a} \sim 200 \mathrm{~m}$

$$
R_{e} \sim 10 \text { ohms }
$$

This reflects effectively as a shunt resistance, so that the effective series resistance would be:

$$
R=\frac{k}{N^{2}} \cdot \frac{Z^{2}}{R_{e}} \sim \frac{.2}{4} \times \frac{80^{2}}{10} \sim 32 \Omega
$$

and $Q \sim 2.5(!)$ 
Effective Q - If we reflect the circuit reactances through this "transformer", they appear as $Z^{\prime}=k \frac{Z}{N^{2}}$ where $k$ is a coupling geometry coefficient ( 2 to .4). Inasmuch as $Z$ will be of order 80 to $100 \Omega$ in our cases, this says that $Z^{\prime} \sim 4 \Omega$, and a closely-coupled alluvium system thus has a $Q \sim \frac{10}{4} \sim 2.5$. It is heavily damped indeed.

The $4 \pi$ Criterion - "Circulating current" models and arithmetic fail badly for $Q$ values less than $\sim 2 \pi$. In practice, it is difficult to design stable (conventional) circuits in which $Q \lesssim 4 \pi$ (for the resonant components). One must turn to non-resonant "conventional" circuits. In the absence of other, more definitive criteria, I will use $Q=4 \pi$ as the specification for heavily-loaded (or broad-banded) resonant sys tems.

This then suggests that one may not want to "couple tighter" (for instance, by burying the loop); if anything, somewhat less coupling may be called for. It also says that buried sensor loops will become "naturally" broad-banded.

The Optimum Illumination Problem

The first exercise was to see what was needed in order to attain a specified field:

$$
B_{C}=\frac{1}{2} \mu_{0}(N I) \frac{a^{2}}{d^{3}} e^{-d / \delta}
$$

This is an axial field definition for $d>>$. We also take $\delta \sim d$. 
Example: $\quad B_{C}=\frac{1}{2} 4 \pi \times 10^{-7}(\mathrm{NI}) \frac{10}{(200)^{3}} e^{-1}$ $\simeq 3.5 \times 10^{-12} \mathrm{NI}$

$$
\text { taking } \begin{aligned}
a & =10 \mathrm{~m} \\
d & =200 \mathrm{~m}
\end{aligned}
$$

1

Thus for $B_{C} \sim 3 \times 10^{-6} \mathrm{Web} / \mathrm{m}^{2} \quad N I=10^{6}$

That's a lot of ampere-turns.

For instance: $10^{4}$ amps and $10^{2}$ turns at $\omega \sim 2 \times 10^{4}$

$$
L \sim \mu_{0} N^{2} a f_{S}=4 \pi \times 10^{-7} \times 10^{4} \times 10 \times 6 \simeq .75 \text { henrys }
$$

Then $Z=\omega L=1.5 \times 10^{4} \mathrm{ohms}$

$$
V \simeq 1.5 \times 10^{8} \text { Volts! }
$$

An alternative is to group these into 10 coils of 10 turns each at $10^{3}$ amps. Then $L \sim .0075$ henrys

$$
\begin{aligned}
& Z=\omega L=150 \Omega \\
& V=1.5 \times 10^{5} \text { Volts which is very high but not incredibly so. }
\end{aligned}
$$

But note the power. Even at $Q \sim 4 \pi$, so that $i \sim \frac{I}{Q} \sim 10^{3}$ amps $\rightarrow 10^{8}$ Watts or 100 Megawatts! Evidently this elementary exercise suggests a different approach. (At $100 \mathrm{~kW}$, we get $\sim 10^{-7} \mathrm{Web} / \mathrm{m}^{2}$ and $2 \times 10^{-3} \mathrm{Web} / \mathrm{m}^{2} \mathrm{sec}$.)

The second exercise was an attempt to simultaneously impose max power and voltage criteria. In particular:

$$
Z=\frac{V_{m}}{I}=\frac{V_{m}^{2}}{Q P}=\omega L_{1} \quad I=\frac{Q P}{V_{m}}
$$

Then $L_{1}=\mu_{0} N^{2} a f_{S}=\frac{V_{m}^{2}}{\omega Q P}$

Determines $N^{2} a=\frac{V_{m}^{2}}{\mu_{0} f_{s} \omega Q P}$

Also $B=\frac{1}{2} \mu_{0}(N I) \frac{a^{2}}{d^{3}}$

(neglecting attenuation). 
This implies that we're better off with large a, small $\mathrm{N}$

$$
B=\frac{2}{2} \mu_{0} N \frac{a^{2}}{d^{3}}\left(\frac{Q P}{V_{m}}\right)
$$

We can write various forms at this point. An interesting one is:

$$
\begin{aligned}
& N_{a}=\frac{V_{m}^{2}}{\mu_{0} f_{s} N \omega Q P} \\
& B=\frac{1}{2} \mu_{0} \frac{a}{d^{3}} \frac{Q P}{V_{m}} \times \frac{V_{m}^{2}}{\mu_{0} N f_{s} \omega Q P}=\frac{V_{m}{ }^{2}}{2 \omega N f_{s} d^{3}}
\end{aligned}
$$

Example: $\quad L_{1}=\frac{10^{8}}{2 \times 10^{4} \times 4 \pi \times 10^{5}}=.004$ henrys

Note also $I \simeq 125$ amps, $Z_{1} \simeq 80 \Omega$

These values will appear throughout the (Alluvium) problem.

$$
N^{2} a=\frac{10^{8}}{4 \pi \times 10^{-7} \times 6 \times 2 \times 10^{4} \times 4 \pi \times 10^{5}} \backsim \underline{\underline{500}}
$$

Thus for

$$
\begin{array}{lll}
N=5 & a=20 & \mathrm{Na}^{2}=2 \times 10^{3} \\
N=1 . & a=500 & \mathrm{Na}^{2}=2.5 \times 10^{5}
\end{array}
$$

But this last case violates the earlier premises -- a $\ll$ d. So we still have work to do.

$$
\text { * } B=\frac{1}{2} \times 4 \pi \times 10^{-7} \times \frac{2 \times 10^{3}}{8 \times 10^{6}} \times 125 \simeq 2 \times 10^{-8} \mathrm{Web} / \mathrm{m}^{2}
$$

Things are improving. 
The Field Optimization Exercise - This is the "full-blush". problem:

We apply the following constraints:

$$
Q=4 \pi \quad P_{\max }, V_{\max } \text { as before }
$$

Axial illumination

$$
\begin{aligned}
& \text { a }>30 \mathrm{~W}^{1 / 3} \quad(250 \mathrm{~m}) \quad \text { ("Save the Coil") } \\
& d=117 W^{1 / 3}(2200 \mathrm{~m})
\end{aligned}
$$

Then:

$$
\begin{aligned}
& B=\frac{1}{2} \mu_{0} N I \frac{a^{2}}{\left(a^{2}+d^{2}\right)^{3 / 2}} \quad I=Q \frac{P_{m}}{V_{m}}=125 \text { amps } \\
& V_{m}=\omega L I \quad \text { and } \quad L=\mu_{0} N^{2} a f_{s}=\frac{V_{m}}{\omega I}=\frac{V_{m}^{2}}{\omega Q P_{m}}=.004 \text { henry }
\end{aligned}
$$

Then $\quad N^{2} a=\frac{V_{m}}{\mu_{0} f_{s} \omega Q P_{m}} \quad N=\frac{1}{\sqrt{a}} \frac{V_{m}}{\sqrt{\mu_{0} f_{s} \omega Q P_{m}}}$

So

$$
B=\frac{1}{2} \sqrt{\frac{\mu_{0} Q P_{m}}{\omega f}} \frac{a^{3 / 2}}{\left(a^{2}+d^{2}\right)^{3 / 2}}
$$

Check Example: (Same numbers as before)

$$
B=\frac{1}{2} \sqrt{\frac{4 \pi \times 10^{-7} \times 4 \pi \times 10^{5}}{2 \times 10^{4} \times 6}} \times \frac{20 \sqrt{20}}{(200)^{3}} \sim 2 \times 10^{-8} \quad \text { "Perfect" }
$$

(The coefficient is $1.8 \times 10^{-3}$ )

Now do

$$
\begin{aligned}
\frac{\partial B}{\partial a} & =\kappa \frac{\partial}{\partial a}\left(\frac{a}{a^{2}+d^{2}}\right)^{3 / 2} \\
& =\kappa \frac{3}{2}\left(\frac{a}{a^{2}+d^{2}}\right)^{\frac{3}{2}} \times\left\{\frac{\left(a^{2}+d^{2}\right)-a(2 a)}{\left(a^{2}+d^{2}\right)^{2}}\right\} \equiv 0
\end{aligned}
$$

This leads at once to $d^{2}-a^{2}=0$. and $\underline{\underline{a} \equiv d}$ at max. field.

$$
\left(\frac{a}{a^{2}+d^{2}}\right)^{3 / 2} \rightarrow\left(\frac{1}{2 d}\right)^{3 / 2}
$$


Another example:

$$
B=\frac{1}{2} \sqrt{\frac{4 \pi \times 10^{-7} \times 4 \pi \times 10^{5}}{2 \times 10^{4} \times 6}} \times \frac{1}{(400)^{3 / 2}} \simeq 2 \times 10^{-7} \mathrm{Web} / \mathrm{m}^{2}
$$

Note $\omega B \sim 4 \times 10^{-3}$ Web $/ \mathrm{m}^{2}$ sec or $4 \mathrm{mv} /$ turn!

So we are able to do almost as well as we started with, using a "not incredible" configuration. 


\section{Receiver Elements}

\section{Sensor Loop Design}

Here we consider L.F. loops of large numbers of turns $N$, with relatively large radii a $(.3 \mathrm{~m}$ to $10 \mathrm{~m})$, and with wire ratios $\left(\frac{\mathrm{a}}{\mathrm{w}}\right)$ in the range $10^{2}$ to $10^{4}$.

$$
L=\mu_{0} N^{2} a\left(\ln \frac{a}{w}-7 / 4\right)
$$

where $w$ is the wire radius or an equivalent dimension $\left(\pi w^{2}=A_{c}\right)$. Consider first the $\ln$ term

$$
f_{\ln }=\ln \frac{a}{w}-7 / 4
$$

$a / w=10^{2} \quad 2 \times 10^{2} \quad 5 \times 10^{2} \quad 10^{3} \quad 2 \times 10^{3} \quad 5 \times 10^{3} \quad 10^{4} \quad 2 \times 10^{14}$

ln $=\begin{array}{llllllll}4.65 & 5.25 & 6.25 & 6.9 & 7.55 & 8.5 & 9.25 & 9.9\end{array}$

$f_{l n}=\begin{array}{llllllll}2.9 & 3.5 & 4.5 & -5.15 & 5.8 & 6.75 & 7.5 & 8.15\end{array}$

In the "interesting" range, $f_{\ell n}$ varies less than a factor of two.

For most evaluation purposes, a "central" value is reasonable to adopt.

I will use $\overline{f_{s}}=6$.

So we write $\underline{L=\mu_{0} N^{2} a f}$
Also

$$
\begin{array}{ll}
R=(2 \pi \mathrm{Na}) \frac{\rho}{\pi \mathrm{W}^{2}} & \text { were } \rho \text { is the wire resistivity } \\
R=\frac{2 \mathrm{~N} \rho \mathrm{a}}{W^{2}} & \text { in particular } \rho_{\mathrm{cu}}=1.7 \times 10^{-8} \mathrm{mks}
\end{array}
$$$$
R=\frac{2 N \rho a}{W^{2}}
$$ 
The loop time constant. This may be determined by external circuit parameters. If we consider only the loop itself, we have $\tau \simeq L / R$ and in particular:

$$
\tau=\frac{\mu_{0} N^{2} a f_{l n} w^{2}}{2 N \rho a}=\frac{1}{2} \mu_{0} N \frac{w^{2}}{\rho} f_{S}
$$

Note that this $\tau$ is (first-order) independent of loop diameter!

Example: $\quad w \sim 10^{-3} \mathrm{~m}$ or $1 \mathrm{~mm}$ and $\mathrm{N} \sim 10^{3}$

$$
\tau=\frac{1}{2} \times 4 \pi \times 10^{-7} \times \frac{10^{3} \times 10^{-6}}{1.7 \times 10^{-8}} \times 6 \sim .2 \mathrm{sec}
$$

Loop resonance. The loop has a self-capacitance $C$ such that it will have a "free" resonance at $\omega=\frac{1}{\sqrt{L C}}$.

This resonance will have an associated decrement characterized by $Q=\frac{\omega L}{R}$. When an inductive element is used in a resonant system, it is generally the practice to make its self-capacitance not more than $v^{\frac{1}{2}}$ the required total capacitance, for reasons of circuit stability, adjustment, etc., (usually much less). In order to make further progress, we need to consider loop self-capacitance features.

Self-Capacitance - Large Sensor Loops. This is treated by considering successive turn layers as equivalent to concentric conductor sheets: Each sheet is $22 w$ thick, and the effective layer is $f_{w} w$, where $f_{w}$ measures 
the combined effect of turn packing and insulation thickness as a fraction of the wire radius $w$. Generally, $f_{w}$ will lie in the range .5 to 1 . We will see that the problem is not too sensitive to

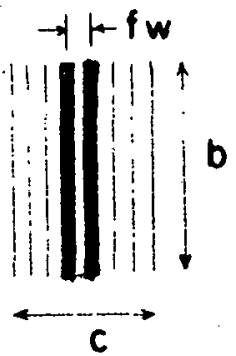
precise choice of this number. Again in the interests of conservative results, I have adopted $f_{w}=1$.

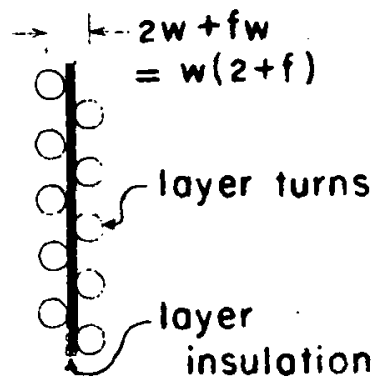

where $k$ is the (effective) dielectric constant for the interspace.

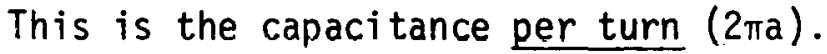

If we approximate the overall reactive shunt consequences by incremental division, then the effective shunt capacitance is

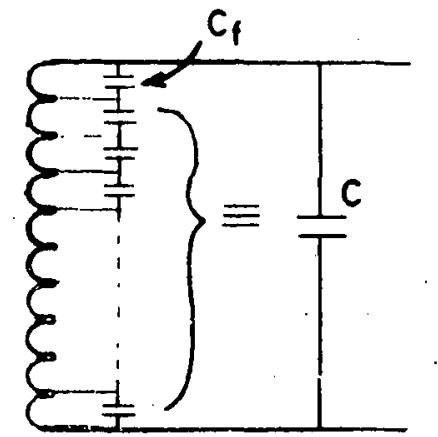

determined by: $\frac{1}{C}=\frac{1}{C_{f}}+\frac{1}{C_{f}}+\ldots=\frac{N^{\prime}}{C_{f}}$

where $N^{\prime}$ counts the number of such sheet condensers. Each 1ayer occupies $w\left(2+f_{w}\right)$ of the coil thickness, as shown, so that $N^{\prime}=\frac{6}{w\left(2+f_{W}\right)}$

Finally: $\quad C=\frac{C_{f}}{N^{\top}}=2 \pi \kappa \kappa_{0} \frac{a b}{f_{w} w^{w}} \times \frac{w(2+f)}{C}=2 \pi \kappa \kappa_{0} \frac{a b}{C}\left(\frac{2+f}{f_{w}}\right)$

If we design so that $b \sim c$ then: $\quad C=2 \pi \kappa_{0} a\left(\frac{2+f_{w}}{f_{w}}\right)$

Note that $C$ is independent of $w$ or $N$ in this model. It only depends on $a$ and on the "packing fraction" $f$. 
Example: (Sensor 10op) $\frac{a}{w}=10^{3} \quad w=10^{-3} \quad a=1 \mathrm{~m}$

Then $\quad C=2 \pi \times 3 \times \frac{10^{-9}}{36 \pi} \times 1 \times\left(\frac{3}{7}\right)=5 \times 10^{-10}$ fds or $\quad 500 \mu \mu \mathrm{fds}$

Also $L=4 \pi \times 10^{-7} \times 10^{6} \times 1 \times 6=2.4 \pi=\underline{7.5 \text { henry }}$

and $\quad R=\frac{2 \times 10^{3} \times 1.7 \times 10^{-8} \times 1}{10^{-6}}=\underline{34 \text { ohms }}$

Resonance occurs at

$$
\begin{aligned}
& \omega=\frac{1}{\sqrt{L C}}=\sqrt{\frac{10^{8}}{.375}} \sim 1.6 \times 10^{4} \text { av or } \quad f \sim 2.5 \mathrm{kHz} \\
& Q=\frac{\omega L}{R} \sim \frac{1.6 \times 10^{4} \times 7.5}{34} \sim 3000 \\
& Z=\omega L=\frac{1}{\omega C} \sim 1.2 \times 10^{5} \text { ohms }
\end{aligned}
$$

This $Q$ is too high to really believe - it only accounts for copper losses in a "DC approximation". This coil is $\sim 3$ " in square section.

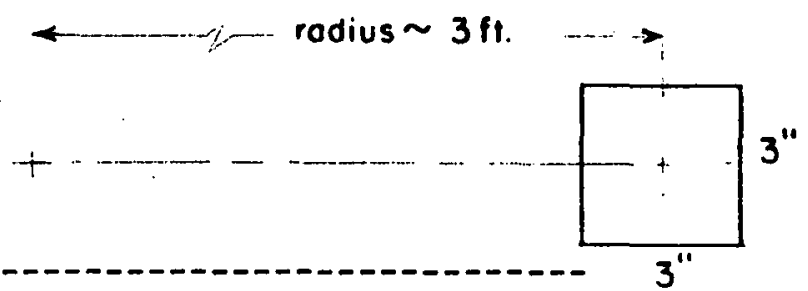

A Multi-Layer Resonance Criterion. We can derive an "inverse" design criterion involving the choice of component resonant frequency. In a "non-resonant" system, it is good practice to locate severe phase/amplitude anomalies well outside the system pass-band - perhaps as much as a factor of three in frequency (with respect to pass-band "edges").. In a "resonant" 
system, we noted elsewhere that the self-capacity of an inductance should be $\frac{1}{2}$ (or less) of the required resonant "tuning" capacitance. Suppose we write in general:

$$
\omega=\frac{1}{\sqrt{L C}} \sim h \omega_{0}=\frac{h c_{0}}{x_{0}} \quad \begin{aligned}
& h \sim 2 \text { or } 3 \text { or so } \\
& \text { (a "sma11 number") } .
\end{aligned}
$$

where $\omega_{0}$ is a criterial frequency - either the system operating frequency, or the edge of the pass-band. Then

$$
\begin{aligned}
& L C \sim \frac{1}{h^{2} \omega_{0}^{2}}=\left[2 \pi \kappa \kappa_{0} a\left(\frac{2+f_{w}}{f_{w}}\right)\right]\left[\mu_{0} N^{2} a f_{s}\right] \\
& L C=\frac{2 \pi \kappa N^{2} a^{2}}{C_{0}^{2}}\left(\frac{2+f_{w}}{f_{w}}\right) f_{S}=\frac{1}{h^{2} \omega_{0}^{2}}
\end{aligned}
$$

where $c_{0}$ is the velocity of light. $x_{0}=\frac{c_{0}}{\omega_{0}}$

Finally

$$
N a=\frac{x_{0}}{h \sqrt{2 \pi \kappa f_{s}\left(\frac{2+f_{w}}{f_{w}}\right)}}
$$

This is an interesting result, as it expresses the coil design requirement in terms of the (air) wavelength.

or also

$$
\omega=\frac{1}{\sqrt{L C}}=\frac{c_{0}}{\sqrt{2 \pi k N a}} \sqrt{\frac{f_{w}}{f_{S}\left(2+f_{w}\right)}}
$$

Note that in the "practical average" case

$$
\sqrt{2 \pi \kappa f_{s}\left(\frac{2+f_{w}}{f_{w}}\right)} \sim \sqrt{2 \pi \times 3 \times 6 \times 4}=12 \sqrt{\pi}=\underline{20}
$$

Thus we write: $\mathrm{Na}=\frac{x_{0}}{20 h} \quad$ (Multi-layer criterion) 
Example: For $x_{0}=1.5 \times 10^{4}$ (corresponding to $\omega=2 \times 10^{4}$ ).

$$
\mathrm{Na} \simeq \frac{1.5 \times 10^{4}}{20 \times 2} \sim \underline{400}
$$

Note that the resonance criteria define the uniqueness between the $\mathrm{Na}$ factor and frequency.

\section{Loop Signals}

Consider the signal induced in a "small" loop due to an arbitrary sinusoidal field B:

$$
V=\pi N \omega a^{2} B \cos \xi
$$

where $\xi$ is the angle made by the fleld vector and the loop axis. We take advantage of this response by virtue of system geometries in which $\xi \rightarrow 0$ with respect to cavity-scattered field terms, and in which $\xi \rightarrow \pi / 2$ in direct generator-coupled fields ("nulling").

Example: Consider a $10^{3}$ turn loop, one $\mathrm{m}$ radius, in a typical signal field $\sim 10^{-10} \mathrm{Web} / \mathrm{m}^{2}(.1$ gamma) at VLF:

$$
V=\pi \times 10^{3} \times 2 \times 10^{4} \times 10^{-10} \simeq .006 \text { volts }
$$

This is the order of signal magnitude with which we are concerned throughout the numbers exercise. Backgrounds are generally $10^{3}$ larger. 


\section{"Fringe" Contribution to Nulled Loops}

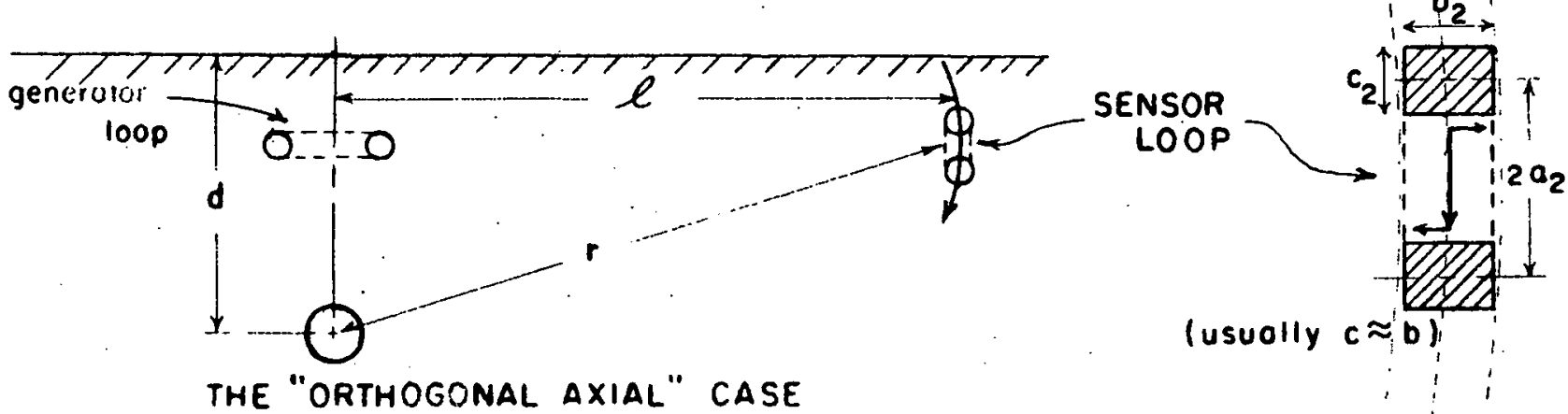

Looking at the various $B$ vectors, the nulling is remarkably good in both crossed loop situations. It is hard to find a first or second-order coupling component. This assumes deliberate provisions for symmetry. For instance, by requiring an even number of layers, so that each pair of layers behaves like a loop turned back on itself. Of course, $b$ is the most critical dimension, and it would appear that in this application, it should be thinner - e.g., $c \sim 2 b$ perhaps.

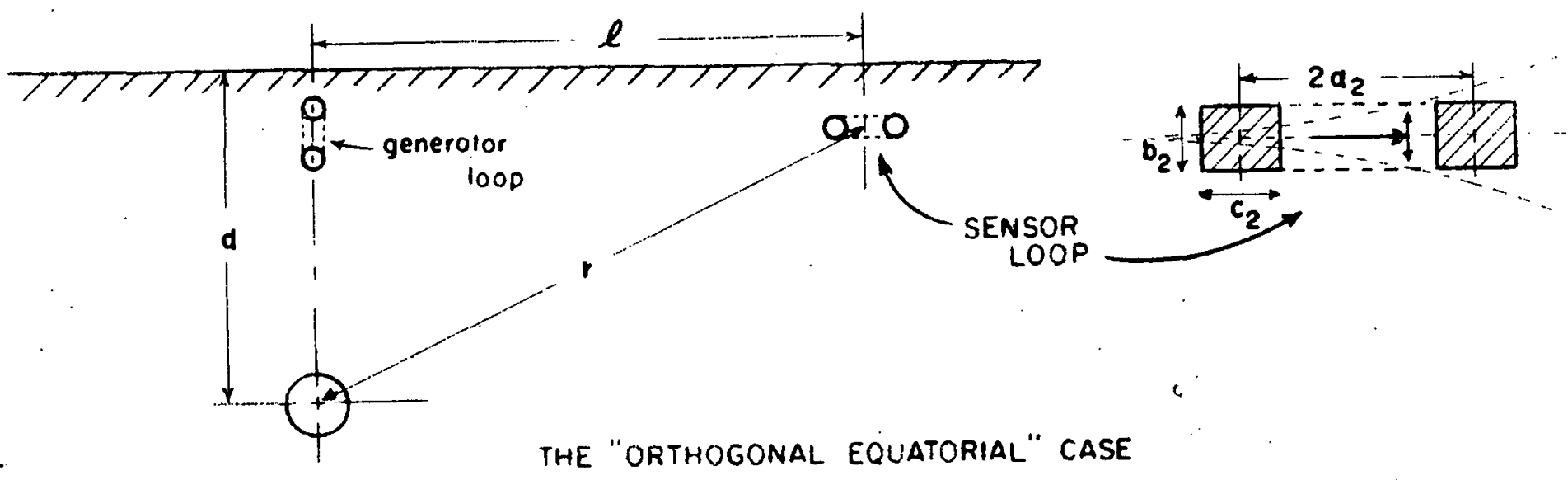


Earth Probe Signals - (High-resistance measuring system)

There are two cases:

Low-conductivity medium, in which case $E=B v_{g}$ and

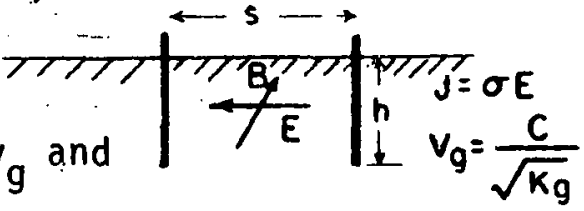
$V=E s=V_{g} B s$.

High-conductivity medium, in which case $E=B v^{\prime}$

and we have to look a bit further:

$$
\delta V=E \delta=\dot{B} \delta A=\omega B \delta^{2}
$$

So

$$
E=\omega B \delta=v^{\prime} B
$$$$
\text { so } \underline{v^{\prime}=\omega \delta}=\frac{\delta}{x_{g}} v_{g}
$$

where $\delta$ is the "skin-depth".

In effect, we say that for $x_{g} \gg \delta$, the local electric field must be related to the magnetic field over a dimension of order $\delta$. Then

$$
V=E s=V^{\prime} B s=\omega B s \delta
$$

Note that $h$ does not enter into these expressions: It will oniy matter when the probes are loaded, since then we must estimate the probe resistance.

\section{Bandwidth Criteria}

As indicated before, $Q$, Power, and Bandwidth are intimately related. If we speak of a bandpass $\Delta \omega$ as determined by the half-amplitude ( $6 \mathrm{db}$ ) response points of a system component, centered at $\omega$, then:

$$
\frac{\Delta \omega}{\omega} \simeq \frac{2}{Q}
$$

If $m$ such components appear serially in a system, the amplitude response at those $\Delta \omega$ points would be $\sim\left(\frac{1}{2}\right)^{m}$. Note that, for $Q \sim 4 \pi: \Delta \omega \simeq \frac{1}{2 \pi} \omega$. 
In order to further specify system response, we should relate bandwidth to some characteristic signal time constant, such as a maximum ( $10 \mathrm{~g})$ rate-of-change or " $\alpha$ ". In a preceding section, the "cavity $\alpha$ " was indicated as one such possibility. $\left(\alpha_{c}=\frac{1}{R} \frac{d R}{d t}\right)$

The Rate-of-Change Criterion. There are several "traditional" hardware criteria for $\alpha$ response:

$$
\left.\begin{array}{l}
\Delta f=\frac{\alpha_{m}}{\pi} \\
\alpha_{m}=\sqrt{\omega_{H} \omega_{L}}
\end{array}\right\} \quad \text { NRL } 4354
$$

These are quite subject to misinterpretation.

There are two cases of interest:

(1) Non-resonant broad-band amplifier $\left\{\begin{array}{l}\omega_{H} \text { is the upper cutoff } \\ \omega_{L} \simeq 0\end{array}\right.$

(2) Tuned broad-band amplifier $\left\{\begin{array}{l}\omega_{H} \\ \omega_{L}\end{array}\right\}=\omega_{0} \pm \delta \omega$

We impose the requirement that an element will "follow" $\alpha$, within. an amplitude fraction $g$, of its infinite bandwidth response. Note "GRF" in NRL 4354.

$$
G R F=\frac{G}{G_{\infty}} \quad g=1-\frac{G}{G_{\infty}} \quad(\text { for small } g)
$$

Thus

$$
\underline{G R F=1-g}
$$
(1) $\operatorname{GRF}=\frac{1}{1+\frac{\alpha}{\omega_{H}}}=1-g$
$1+\frac{\alpha}{\omega_{H}}=\frac{1}{1-g}$

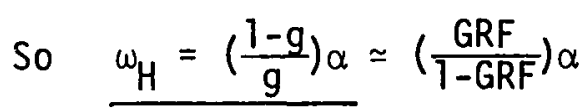
Also see figure 7 in NRL $4354(n=1)$ 
The GRF for $\omega_{H}=\alpha$ is .5

$$
\text { for } \omega_{H}=2 \alpha \text {, it is } .67 \text { (corresponds to } \Delta f=\frac{\alpha_{m}}{\pi} \text { ) }
$$

(Note that $\omega_{H}$ is defined as the " $3 \mathrm{db}$ " point.)

(2) The "tuned" case is really identical to the non-resonant case, as can be seen by considering the single-sideband model. For it, $\delta \omega \sim \omega_{H}$, etc. Thus:

$$
\delta \omega \simeq\left(\frac{l-g}{g}\right) \alpha=\frac{1}{2} \Delta \omega
$$

Example: Suppose $g=.1$ (10\% "error") then:

$$
\begin{aligned}
\omega_{H}=\delta \omega \simeq \frac{.9}{.1} \alpha & =9 \alpha \quad(!) \\
\delta f & =\frac{9 \alpha}{2 \pi} \sim 1.5 \alpha \quad \text { (This is severe }
\end{aligned}
$$

Note that it is not $\frac{\alpha}{\pi}$ :

Correspondence with the resonance criterion. If a self-resonant $\left(\omega_{0}\right)$ element appears in a non-resonant system, then it is usually desirable for $\omega_{0} \gg\left\{\begin{array}{c}\Delta \omega \\ \omega_{H}\end{array}\right\}$, by about the same-factor as indicated for resonant "tuning": $\omega_{\mathrm{O}} \sim h \omega_{H}$.

Center frequency criterion. The minimum "carrier" frequency follows immediately:

$$
\omega_{\min }={ }^{1} 0_{\min } \Delta \omega=9 \alpha Q_{\min }
$$

For $\alpha_{c} \sim 500$, and $Q_{\min } \sim 4 \pi$, find $\omega_{\min } \sim 6 \times 10^{4}$ ! 
Sensor Example (1): Suppose $h=2 . \quad x_{0}=\frac{3 \times 10^{8}}{2 \times 10^{4}}=1.5 \times 10^{4}$

$$
\begin{aligned}
& k=3 \quad f_{s}=6 \quad f_{w}=1 \\
& N a=\frac{1.5 \times 10^{4}}{2 \sqrt{2 \pi \times 3 \times 6 \times 3}} \sim \underline{400}
\end{aligned}
$$

For a $\sim 1$ met, we can afford $\sim 400$ turns by this criterion.

Sensor Example (2): $\quad(10 \%)$ Broadband system for which $\Delta \omega \sim \omega_{H} \sim 1.5 \alpha$

$$
\text { Take } \quad \alpha \sim 10^{3} \quad \lambda_{0}^{1} \simeq \frac{3 \times 10^{8}}{1.5 \times 10^{3}}=2 \times 10^{5}
$$

(Everything else the same)

Since $\mathrm{Na} \propto \ddot{\lambda}_{0}$, we have at once

$$
\mathrm{Na}=400 \times \frac{2 \times 10^{5}}{1.5 \times 10^{4}} \sim \underline{5000} \text { and so forth }
$$

The Optimum Coupling Problem (Dipole Field)

We want to find the effective signal field component at an arbitrary location $(r, \theta)$ with orthogonal element orientations.

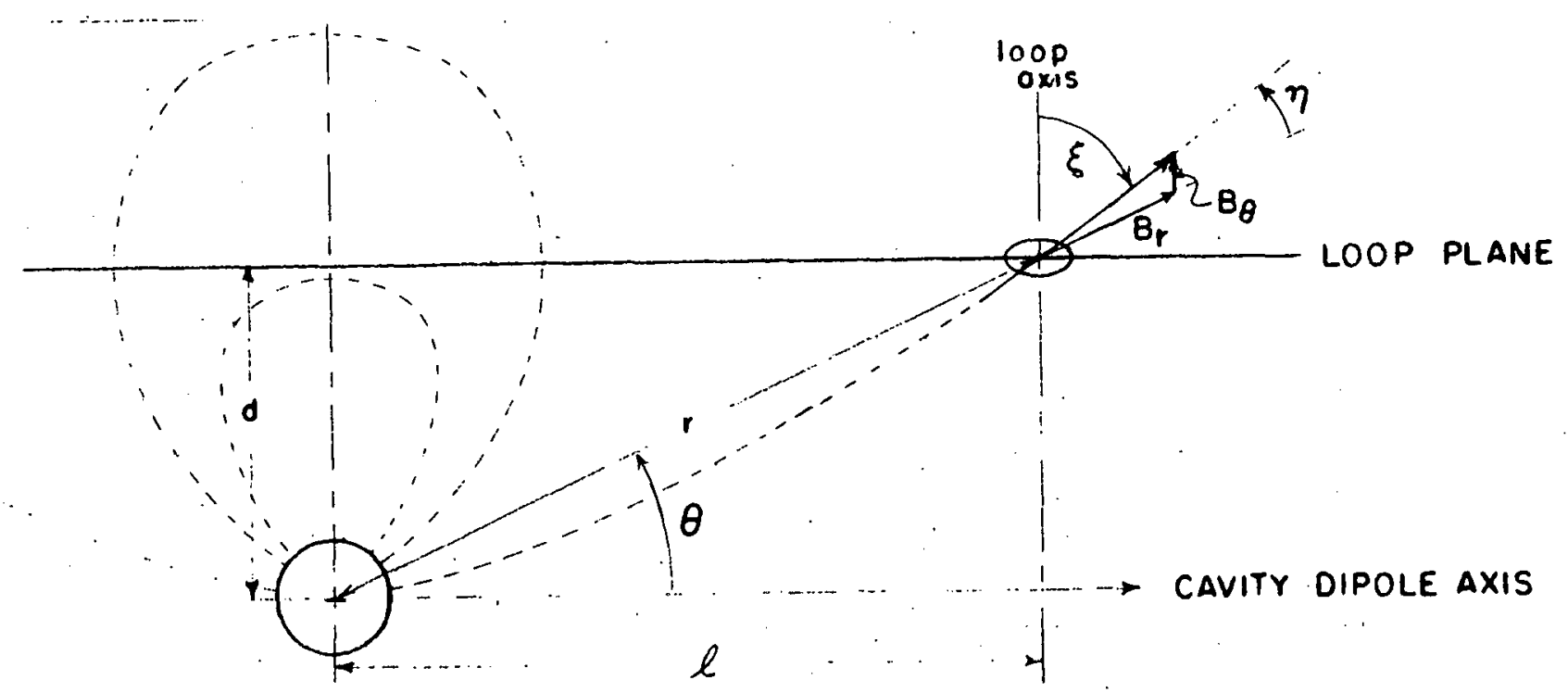




$$
B=\sqrt{B_{r}^{2}+B_{\theta}^{2}}=\frac{\mu_{o} m}{4 \pi} \frac{1}{r^{3}} \sqrt{2 \cos ^{2} \theta+\sin ^{2} \theta}
$$

Note that $\quad \eta=\tan ^{-1} \frac{B_{\theta}}{B_{r}}=\tan ^{-1}\left(\frac{1}{2} \tan \theta\right)=\tan ^{-1} \frac{1}{2} d / \ell$

$$
\theta=\tan ^{-1} \mathrm{~d} / \ell
$$

Then

$$
\xi=\pi / 2-(\eta+\theta)=\pi / 2-\left(\tan ^{-1} d / \ell+\tan ^{-1} d / 2 \ell\right)
$$

and

$$
B_{\text {eff }}=B \cos \xi \quad \text { as so defined }
$$

The Interception Optimization Problem

$$
\cos \xi=1 \text { for } \theta+n=\pi / 2
$$

Horizontal Dipole Axis

$$
\tan ^{-i}\left(\frac{d}{\ell}\right)+\tan ^{-1}\left(\frac{d}{2 \ell}\right)=\pi / 2
$$

Vertical Dipole Axis simply reverses $d$ and $\ell$

$$
\tan ^{-1}\left(\frac{\ell}{d}\right)+\tan ^{-1}\left(\frac{\ell}{2 d}\right)=\pi / 2
$$

We write the problem as $\tan ^{-1}(2 x)+\tan ^{-1} x=\pi / 2$

where $X$ can be either ratio.

$$
\begin{aligned}
& \text { At } \psi_{1}=34.3^{\circ} \\
& \tan \psi_{1}=.707=\sqrt{2} / 2 \\
& \psi_{2}=54.7^{\circ} \\
& \tan \psi_{2}=1.414=\sqrt{2}
\end{aligned}
$$

Thus $\left.\begin{array}{l}\ell / d \\ d / \ell\end{array}\right\}=\sqrt{2}$ defines the locations providing maximum coupling.
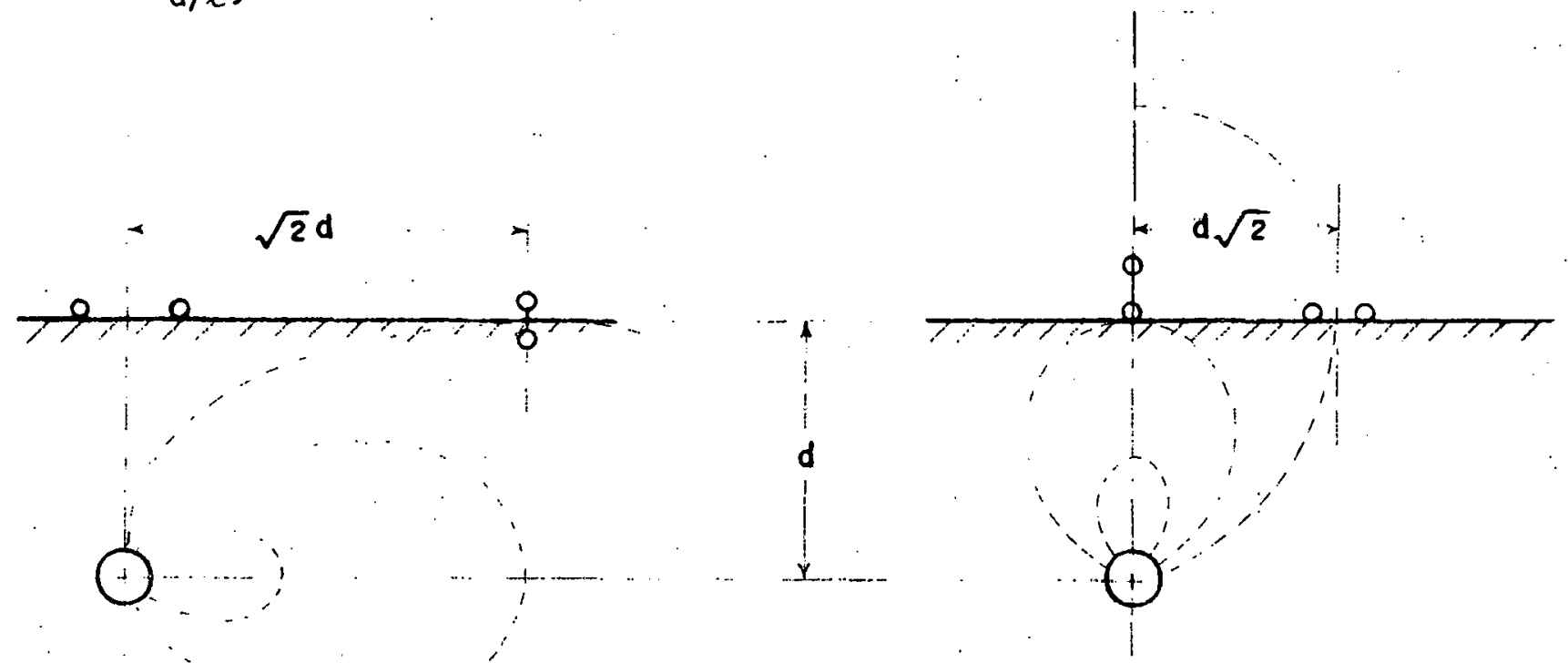
Radiative Field Descriptions

Topics included here:

Radiative Dipole Expressions

Attenuation

Power-Radiation Resistance

-. An Induced Current Argument

In this frequency domain, bandwidth is a relatively trivial consideration. It comes "naturally".

For reasons of expediency, I have used the infinitesimal dipole approximations to describe generator and sensor elements. For a given radiative power, this provides representative answers. Clearly if one goes to finite elements involving phased arrays, it should be possible to "do better".

The Dipole Equations in the Radiative Limit (MF and HF):

Current Dipole $\quad E_{\theta}=\left(\frac{\mu_{o} p}{4 \pi}\right)\left(\frac{v g}{x_{g}}\right)\left(\frac{\sin \theta}{r}\right)=v_{g} B_{\phi}$

$$
B_{\phi}=\left(\frac{\mu_{0} P}{4 \pi}\right)\left(\frac{l}{x_{g}}\right)\left(\frac{\sin \theta}{r}\right)
$$

Magnetic Dipole $\quad B_{\theta}=\left(\frac{\mu_{0}^{m}}{4 \pi}\right)\left(\frac{1}{x_{g}^{2}}\right)\left(\frac{\sin \theta}{r}\right)$

$$
E_{\phi}=\left(\frac{\mu_{0}^{m}}{4 \pi}\right)\left(\frac{v_{g}}{x_{g}^{2}}\right)\left(\frac{\sin \theta}{r}\right)=v_{g} B_{\theta}
$$




\section{Attenuation}

I characterize an "EM mfp" or diffusion length in alluvium by the "skin depth": $\delta=\sqrt{\frac{2}{\mu_{0} \sigma \omega}}$ and in granite by the frequency-independent length: $\quad \gamma=\frac{2}{\sigma} \sqrt{\frac{k_{g^{k}}}{\mu_{0}}}$. Somewhat arbitrarily, I introduce absorption by virtue of $\mathrm{e}^{-r / x}$ in the two cases, respectively. This is "exact" for radiative limit problems. It is an approximation in the VLF domain for which $x_{g} \gg \delta$; the error is generally no greater than a factor of two.

Power Req't - Radiative Case (Granite only)

We only consider "small" source elements (i.e., $d \ll x_{g}$ ). We also neglect losses in the medium (i.e., $d \ll \gamma$ also). First notice the propagation impedance in earth:

$$
z_{g}=\sqrt{\frac{\mu_{o}}{\kappa_{g} \kappa_{o}}}=\frac{1}{\sqrt{\kappa_{g}}} z_{0}=1 / 3 \times 377 \simeq 126 \Omega
$$

Small Loop of moment $m=\pi a^{2} \mathrm{NI}$

Peak Power: $\quad P=\frac{Z_{0} m^{2}}{12 \pi \sqrt{K_{g}} \lambda_{g}^{4}}=\frac{\pi}{12} Z_{g}\left(\frac{a}{\lambda_{g}}\right)^{4} N^{2} I^{2}$

Rad Res: $\quad R=\frac{2 P}{I^{2}}=\frac{\pi}{6} Z_{g}\left(\frac{a}{X_{g}}\right)^{4} N^{2}$

Current Element of current moment $p=I \ell$

Peak Power $\quad P=\frac{1}{12 \pi} z_{g}\left(\frac{l}{x_{g}}\right)^{2} I^{2}=\frac{z_{g}}{12 \pi x_{g}^{2}} p^{2}$

Rad Res: $\quad R=\frac{2 P}{I^{2}}=\frac{Z g}{6 \pi}\left(\frac{l}{x_{g}}\right)^{2}$ 


\section{An Induced Current Argument; Cavity Moments}

I continue to assume that the elements (generator, cavity, sensor) are dimensionally small compared to the wavelength. In particular, I can use the "immersion" expressions provided that a current density can be defined. To do this, I use the displacement current, writing:

$$
J=\kappa_{g} \kappa_{0} \frac{\partial E}{\partial t}=\kappa_{g} \kappa_{0} \omega E .
$$

Then

$$
p=4 \pi R^{3} J=\left(4 \pi R^{3}\right)\left(\kappa_{g} \kappa_{0} \omega\right) E
$$

Also.

$$
m=\left(4 \pi R^{3}\right) \frac{1}{\mu_{0}} B
$$

It is easily seen that these are the same thing:

$$
\begin{aligned}
P_{p} & =\frac{Z_{g}}{12 \pi x_{g}^{4}} p^{2}=\frac{Z_{g}}{12 \pi x_{g}^{2}}\left(4 \pi R^{3}\right)^{2}\left(\kappa_{g} K_{0} \omega E\right)^{2} \\
& =\frac{Z_{g}}{12 \pi x_{g}^{2}}\left(4 \pi R^{3}\right)\left(\kappa_{g} \kappa_{o} \mu_{0}\right)^{2} \omega^{2} \frac{\left(v_{g}{ }^{B}\right)^{2}}{\mu_{o}^{2}}=\frac{Z_{g}}{12 \pi x_{g}^{2}}\left(\frac{\omega}{v_{g}}\right)^{2}\left(4 \pi R^{3}\right)^{2}\left(\frac{B}{\mu_{o}}\right)^{2} \\
& =\frac{Z_{g}}{12 \pi x_{g}^{4}} m^{2}=P_{m}
\end{aligned}
$$




\section{THE PROTOTYPE CASES}

Having so laboriously set the stage, the selection and evaluation of an array of representative (but distinguishable) cases is almost consequential. In actual fact, there was much less causality in this development, than this neat organization implies.

The examples calculated here include both singular schemes and typifications of broad classes. There is nothing particularly special about the chosen dimensions and other non-specific features. It would be surprising to find that nominal variations within each class had any significant consequence.

These estimates make use of the various model descriptions outlined in the previous section. They are believed to be good to better than an order of magnitude, and some numbers are probably good to a factor of two for the assumed problem. It is important to recognize that actual test geology, "real" instrumentation and other significant practical factors, would not reproduce the case idealizations shown by the figures. Thus, greater precision in problem description and solution would not be warranted, without much more elaborate detailing of "real" circumstances.

Reference should be made to Table II for a summary of the results obtained here and for direct comparison. 
The Conduction Schemes (Figure 3)

These configurations are restricted to relatively high-conductivity media, such as alluvium and tuff, and to DC and VLF energy injection. (Because of the system dimensions.) The basic idea is that the lowresistance cavity plasma introduces a "current concentration" element, which generates a magnetic field whose time history is related to the cavity growth.

In the D.C. case, this signal is sensed as a real-time rate-of-change in the surface magnetic field. There is no "carrier" in this singular problem. Inasmuch as the steady-state (pre-detonation) condition has no rate-of-change, the $S / B$ ratio is essentially infinite. However, we will see that the signal amplitude itself is discouragingly small, and that overwhelming competition arises from several other noise mechanisms. The required environmental control is prohibitively difficult and I evaluate this scheme as technically unfeasible.

In the VLF case, the carrier removes the cavity modulation from the principal noise domain, although the question remains open as to the possible interaction of several of the noise generators with the VLF field. This is only a slightly less unattractive scheme.

Aside from the formidable noise problems, these schemes have several other difficulties. The geometry is idealized as a pair of buried half-cylinders, as used in the analytic model above. In practice, it would more likely consist of a number of vertical casings 
connected in parallel rows. In either case, there is a severe (VLF) current supply problem, in that the inductive reactance of those lines can easily be comparable to the earth resistance. Hence one would have to go to some effort to minimize this, by deliberate conductor layout and parallel feed cables. In turn, this could easily create a severe local background problem in the sensor vicinity.

That problem would not exist in the D.C. case. Instead, it is replaced by noise from power supply ripple (generator commutation, rectifier switching, etc.). The allowable (and almost preposterously smal1) limit for such noise fields will also be indicated.

We abandon these dark thoughts, in the pursuit of numbers. Of course, we use the prototype cavity in "typical" alluvium.

The prototype sensor in both cases will be a many-turn loop placed above ground. Evidently its plane must be parallel to the current direction in order to couple to the cavity dipole. This means that it will also couple to the "diffuse" background field due to the exciting current itself. In the VLF case, this leads to a poor signal/background number, but not hopelessly so. 


\section{The D.C. Conduction Case}

Cavity Illumination: Current density -

$$
\mathrm{J}_{0}=\frac{2 \mathrm{I}}{\pi \mathrm{L}} \frac{\ell}{\ell^{2}+\mathrm{d}^{2}}
$$

Current Dipole:

$$
p=4 \pi R^{3} J_{0}
$$

Signal Field:

$$
B_{S}=\left(\frac{\mu_{0} p}{4 \pi}\right) \frac{\sin \theta}{d^{2}}
$$

Signal:

$$
S=\left(\pi N a_{2}^{2}\right) \frac{\partial B_{S}}{\partial t} \cos \xi
$$

Rate-of-change:

$$
\begin{aligned}
\frac{\partial B}{\partial t} & =B_{S}\left(\frac{1}{p} \frac{\partial p}{\partial t}\right)=B_{S} \frac{1}{R^{3}} \frac{\partial}{\partial t}\left(R^{3}\right) \\
& =3 B_{S}\left(\frac{1}{R} \frac{\partial R}{\partial t}\right)=3 \alpha_{c} B_{S}
\end{aligned}
$$

Then:

$$
S=\left(3 \pi N_{2} a_{2}^{2}\right) \alpha_{c}\left(\frac{\mu o}{4 \pi}\right)\left(\frac{\sin \theta}{d^{2}}\right) \cos \xi\left(4 \pi R^{3}\right)\left(\frac{2 I_{1}}{\pi L}\right)\left(\frac{l}{\ell^{2}+d^{2}}\right)
$$

Find:

$$
S=\left(6 \mu_{0} N_{2} I_{1} \alpha_{c}\right)\left(\frac{a_{2}^{2} \ell d}{L\left(\ell^{2}+d^{2}\right)}\right) k^{3} \sin \theta \cos \xi
$$

This signal depends on $\alpha_{c} k^{3}$. These don't maximize together, obviously. Figure 2 also has this function plotted, and its maximum value is about .01 per sec.

Signal Numbers: Since the load resistance was pegged at $8 \Omega$, then: $P=I^{2} R=10^{5}$ watts which leads to $I_{1} \simeq 110$ amps and $V \sim 880$ volts. These are reasonable numbers.

The Sensor is specified by $h=2, x_{\alpha}=\frac{c_{0}}{a_{m}} \sim \frac{3 \times 10^{8}}{1.5 \times 10^{3}} \sim 2 \times 10^{5} \mathrm{~m}$.

Then:

$$
N_{2} a_{2}=\frac{x_{\alpha}}{20 h} \sim \frac{2 \times 10^{5}}{40}=5 \times 10^{3}
$$


Evidently we're better off with large $a_{2}$ and small $N_{2}$. We choose $a_{2}=5 \mathrm{~m}, \mathrm{~N}_{2}=10^{3}$.

and

$$
S=\left(6 \times 4 \pi \times 10^{-7} \times 10^{3} \times 110 \times .01\right)\left(\frac{25 \times(200)^{2}}{200 \times 2 \times(200)^{2}}\right)=5 \times 10^{-4} \text { Volt }
$$

That's not many volts out of such a large coil!

Noise

Suppose we first estimate what it takes to make "competitive" Noise:

$$
\begin{gathered}
V=\pi N_{2} a_{2}^{2} \alpha^{\prime} B \\
\alpha^{\prime} B \simeq \frac{V}{\pi N_{2} a_{2}^{2}} \sim \frac{5 \times 10^{-4}}{\pi \times 10^{4}} \sim 10^{-8} \mathrm{Web} / \mathrm{m}^{2} \mathrm{sec}
\end{gathered}
$$

Considering that 1 gamma is $10^{-9} \mathrm{Web} / \mathrm{m}^{2}$, then at $\omega \sim 377$ (60 cps), we have $\alpha B \simeq 3.8 \times 10^{-7} \mathrm{Web} / \mathrm{m}^{2} \mathrm{sec}$. Thus the signal is comparable to a .03 gamma power field:

This criterion gives us a measure of competition for other noise generators:

$$
\begin{array}{ll}
\text { Power Supply } & \text { Earth Motion } \\
\text { Coil Motion } & \text { Casing Current }
\end{array}
$$

Power Supply - We look ahead a bit to obtain the expression:

$$
N \simeq\left(\mu_{0} N_{2} i_{1} \omega\right)\left(\frac{a_{2}^{2} d}{L l}\right)
$$

where $i_{1}$ is the power source ripple current at frequency $w$.

Numerically, find $N \simeq\left(4 \pi \times 10^{-7} \times 10^{3} \times i_{1} \omega\right)\left(\frac{25 \times 200}{(200)^{2}}\right)$

$$
=500 \pi \times 10^{-7} i_{1} \omega=5 \times 10^{-4} \quad \text { for unity } S / B
$$


For instance, if the power supply as a 360 cycle comutation ripple, then: $\quad i=\frac{5 \times 10^{-4} \times 10^{7}}{500 \pi \times 2 \pi \times 360} \simeq .0015 \mathrm{amp} \quad$ (out of $2100 \mathrm{amps}$ )

So the power supply ripple must be held down to $2.001 \%$.

Power Cable - Consider a single nearby power cable (long loop return). Its field rate is given by:

$$
\omega B=\frac{\mu_{0} i}{2 \pi r} \omega \simeq 10^{-8} \quad \text { (for } S / N=1 \text { ) }
$$

Then $\quad \frac{i}{r}=10^{-8} \times \frac{2 \pi}{4 \pi \times 70^{-7}} \times \frac{1}{377}=1.5 \times 10^{-4} \mathrm{amp} / \mathrm{met}$

At 10 meters, a 60 cycle power cable carrying one milliampere of "unbalanced" current produces as large a signal as the cavity!

Coil Motion - In the earth's field $\left(\sim 10^{-4} \mathrm{Web} / \mathrm{m}^{2}\right)$, we estimate the competitive rate of motion, writing:

$$
S=\left(\pi N_{2} a_{2}^{2}\right) \alpha_{L} B_{e} \cos \xi
$$

where $\alpha_{L}=\frac{1}{a} \frac{d x}{d t}$ measures the coil's motion in terms of its dimension.

$$
S=\left(\pi \times 10^{3} \times 25\right) \alpha_{L} \times 10^{-4} \times .7=5 \times 10^{-4} \text { again. }
$$

Find

$$
\alpha_{L}=10^{-4} \text { per sec. }
$$

Hence $\frac{d x}{d t}=10^{-4} \mathrm{a}=5 \times 10^{-4} \mathrm{~m} / \mathrm{s}$.

The coil's velocity may never exceed $\sim .05 \mathrm{~cm} / \mathrm{sec}$.

Its acceleration can be estimated by invoking that the worst competitive signal is one that looks like the cavity signal, for which $\alpha \sim 100 / \mathrm{sec}$. Therefore the maximum permissible acceleration is $\sim 5 \mathrm{~cm} / \mathrm{sec}^{2}$ or $.005 \mathrm{G}$ (for unity signal/noise).

Earth Motion - The worst earth motion situation is that due to strong shock, for which $\frac{\Delta x}{\Delta t} \sim 500$. This induces a current in the medium measured by:

$$
J=\sigma E=\sigma v_{s} B \text { e } \cos \xi \simeq \frac{500 \times .7 \times 10^{-4}}{400} \sim 10^{-4} \mathrm{amps} / \mathrm{m}^{2}
$$


That's competitive with the excitation current, and we find:

$$
B_{S} \simeq \frac{1}{2} \mu_{0} I_{S}
$$

where $I_{s}$ is the effective surface current density.

We take $\quad I_{S}=J v_{s h} \Delta t \sim 10^{-4} \times 2 \times 10^{3} \times 10^{-3} \sim 2 \times 10^{-4} \mathrm{amps} / \mathrm{m}$

( $v_{s h} \Delta t$ is a characteristic shock length)

So

$$
B_{S} \sim 10^{-10} \text { Web/m }
$$

Then

$$
\begin{aligned}
N & =\left(\pi a_{2}^{2} N_{2}\right) B_{s} / \Delta t \\
& =\left(\pi \times 25 \times 10^{3}\right) \times 10^{3} \times 10^{-10}=.01 \text { volt }
\end{aligned}
$$

This noise source leads to $S / N \sim .05$.

Casing Current - Consider an initial casing current pulse of order 3000 amps and $\omega \sim 2000$ (at the top end only).

$$
\omega B \simeq \frac{\mu_{0} I c a}{2 \pi l} \omega
$$

and

$$
\begin{aligned}
N & =\left(\pi a_{2}^{2} N_{2}\right) \omega B=\frac{1}{2}\left(\mu_{0} N_{2} I_{c a} \omega\right) \frac{a_{2}^{2}}{l} \\
& =\frac{1}{2}\left(4 \pi \times 10^{-7} \times 10^{3} \times 3 \times 10^{3} \times 2 \times 10^{3}\right) \times \frac{25}{200} \\
& =150 \pi \sim 500 \text { Volts! } \quad \text { So } N_{f} \sim 10^{6}
\end{aligned}
$$

Evaluation - As thereby implied, the required environmental control is prohibitively difficult, and I evaluate this scheme as technically and programmatically unfeasible. 


\section{The VLF Conduction Case}

The signal expressions are the same (as the $D C$ case) down to the rate-of-change definition, which now becomes simply:

$$
\frac{\partial B_{s}}{\partial t}=\omega B_{s}
$$

We should also include a somewhat arbitrary attenuation factor and a $Q$ resonance factor:

Then: $\quad S=\left(\pi N_{2} a_{2}^{2}\right) \omega\left(\frac{\mu_{0}}{4 \pi}\right)\left(\frac{\sin \theta}{d^{2}}\right) \cos \xi\left(4 \pi R^{3}\right)\left(\frac{2 I_{1}}{\pi L}\right)\left(\frac{l}{l^{2}+d^{2}}\right) Q_{2} e^{-2 d / l}$

and $S=\left(2 \mu_{0} N_{2} I_{1} \omega\right)\left(\frac{a_{2}^{2} l d}{L\left(\ell^{2}+d^{2}\right)}\right) k^{3} Q_{2} e^{-2 d / \delta}$

This signal depends only on $k^{3}$ (and hence on how late the plasma remains meaningfully an "infinite conductor").

Background Field - This is derived as an approximation, using

$$
E=\frac{J_{s}}{\sigma}=v^{\prime} B_{b} \sim \omega \delta B_{b}
$$

so that

$$
\begin{gathered}
B_{b} \simeq \frac{J_{s}}{\sigma \omega \delta}=\frac{1}{2} \mu_{0} \\
J_{s}=\frac{2 I}{\pi L l}
\end{gathered}
$$

Background -

$$
\begin{aligned}
B & =\left(\pi N_{2} a_{2}^{2}\right) \omega B_{b} Q \\
& =\left(\pi N_{2} a_{2}^{2}\right) \omega\left(\frac{1}{2} \mu_{0} \delta\right)\left(\frac{2 I_{1}}{\pi L \ell}\right) Q
\end{aligned}
$$

and

$$
B=\left(\mu_{0} N_{2} I_{1} \omega\right)\left(\frac{a_{2}^{2} \delta}{L \ell}\right) Q_{2}
$$


A key number in this exercise is the frequency itself. It will be specified in all of the VLF examples by:

$$
\delta=\sqrt{\frac{2}{\mu_{0} \sigma \omega}} \simeq d=200 \mathrm{~m}
$$

This leads to $\omega=2 \times 10^{4}$ or about $3.3 \mathrm{kHz}$.

The superficial difficulty with this "carrier" frequency is that it hardly appears sufficient to support the signal modulation imposed by the bandwidth criteria. There are several ways around this, such as single-sideband system design, response compensation (elsewhere), or higher frequency. (Doubling w hardly changes our arguments at all, since $\omega e^{-d / \delta}$ is almost a constant in this domain.) We'll stick with it for the purposes of this study.

Signal Numbers: Again we use the same power input assumptions:

$$
\begin{array}{ll}
\mathrm{P}=10^{5} \text { Watts } & \mathrm{R}=8 \Omega \\
I=110 \mathrm{amps} & \mathrm{V}=880 \mathrm{~V}
\end{array}
$$

We also have

$$
\omega=2 \times 10^{4} \quad x=1.5 \times 10^{4} \mathrm{~m}
$$

Here I use the sensor loop specified for the next (VLF) case:

$$
\begin{gathered}
a_{2}=5 \mathrm{~m} \quad N_{2}=80 \\
S=\left(2 \times 4 \pi \times 10^{-7} \times 80 \times 110 \times 2 \times 10^{4}\right)\left(\frac{25 \times(200)^{2}}{200 \times 2 \times(200)^{2}}\right) \times 10^{-3} \times 4 \pi \mathrm{e}^{-2}=.05 \text { Volt }
\end{gathered}
$$

This is a relatively "strong" signal. 
Signa $]$ /Background

$$
S / B=2\left(\frac{l^{2}}{l^{2}+d^{2}}\right)\left(\frac{d}{\delta}\right) e^{-2 d / \delta} k^{3}
$$

Inasmuch as $\omega$ is chosen so that $d \sim \delta$, and also $\ell \sim d$, then:

$$
S / B+e^{-2} k^{3} \sim \underline{1 / 7 \times 10^{-3}} \quad \underline{N_{f} \sim 7000}
$$

This is an interesting result. It is not "hopeless" since no auxiliary background suppression has yet been imposed on the system. In particular, there are two things we could do to treat the sensor vicinity:

Install a local group of earth electrodes through which a cancelling current can be passed to achieve zero surface current.

Install a guard loop system adjusted to null out the residual background field at the sensor. Since this is poorly coupled to the cavity, it should not noticeably affect that signal.

In addition, one can always inject further "trace" signals in the equipment itself for steady-state nulling purposes.

Evaluation. Nevertheless, I evaluate this scheme as unattractive from a programmatic standpoint, and technically marginal. 


\section{The Quasistatic Magnetic Field Schemes (Figure 4 thru 6)}

We consider three specific cases whose analysis appears to be valid for both media: 400 ohm-m alluvium and $10^{4}$ ohm-m in granite (as well as anything in between). As illustrated, the basic idea is to immerse the cavity in a VLF magnetic field, and to observe its perturbation as a re-radiated component. The first case resembles the original "spontaneous" early concept for the entire scheme, as mentioned earlier under frequency criteria. That is, simply to place a transmitter loop and a receiver loop somewhere near the event and "see what happens".

These three specific cases were chosen to be close to geometrical optimums in each configuration sub-class:

1. Axial coupling with orthogonal elements.

2. Axial coupling with concentric elements.

3. Equatorial coupling with orthogonal elements. It will be noted that all three cases show the generator element as being placed directly above the detonation point. Evidently there are innumerable other choices of location and orientation. Some of these were also considered, but none revealed anything unique relative to the chosen geometry. They did confirm the optimization requirement that one or the other element occupy that central position. The choice of generator element is a consequence of the pragmatic symmetry criterion discussed earlier, as well as of the economic penalties associated with off-center generator loops. For the axial cases, the optimum generator loop would neatly surround the subsidence crater. In principle, it could then be reusable. 
The numbers are obtained for the alluvium case; granite can be simply extrapolated by omitting the attenuation term.

1. Axial Illumination, Orthogonal Loops. ("End-Fire")

The "end-fire" schemes involve the use of a large, resonant, field generator loop whose axis passes through the detonation point. The optimization exercise (see "Descriptions") indicated that maximum coupling for such a geometry obtains with $a_{1} \simeq d$. This is adequately larger than the crater, of course.

This sub-class uses a sensor loop oriented so that its axis is orthogonal to the generator loop, and located for optimum cavity field interception. (i.e., $\cos \xi \sim 1$ )

Physically, the generator loop is envisioned as consisting of a copper bus-bar (of moderately large cross-section $\sim .01 \mathrm{~m}^{2}$ ) mounted on a circle of sunken concrete block anchors (by means of insulators). The sensor loop would be an interlaced, multi-layer winding of large stranded conductor, plastic-cast into a rigid doughnut form. 
Generator Loop Specs.

$$
\text { Criteria: } \begin{aligned}
P & =I_{1}^{2} R_{1}=10^{5} \mathrm{~W} \\
V_{m} & =I_{1} Z_{1}=10^{4} \mathrm{~V} \\
Q_{1} & =\frac{Z_{1}}{R_{1}}=4 \pi \\
\omega & =2 \times 10^{4} \\
x & =1.5 \times 10^{4} \mathrm{~m}
\end{aligned}
$$

Find: $\quad Z_{1}=\omega L_{1}=80 \Omega$

$$
\begin{aligned}
& R_{1}=\frac{Z_{1}}{Q}=6.4 \Omega \text { equiv. } \\
& I_{1}=125 \text { amps } \\
& L_{1}=4 \times 10^{-3} \text { hen. } \\
& C_{1}=.67 \text { ufd equiv (!) }
\end{aligned}
$$

Also: $\quad d=200 \mathrm{~m}$

$$
a_{1}=200 \mathrm{~m}
$$

Inductance criterion leads to:

$$
\begin{aligned}
L_{1} & =\mu_{0} N_{1}^{2} a_{1} f_{S} \quad \text { where } f_{s} \simeq 6 \\
\text { or: } \quad N_{1} & =\sqrt{\frac{L_{1}}{\mu_{0} a_{1} f_{S}}}=\sqrt{\frac{4 \times 10^{-3}}{4 \pi \times 10^{-7} \times 200 \times 6}} \sim 2 \text { turns (more-or-7ess) }
\end{aligned}
$$

Finally, the loop moment is:

$$
m_{1}=\pi a_{1}^{2} N_{1} I_{1}=\underline{3 \times 10^{7}}
$$




\section{Sensor Loop Specs.}

Criteria: $h=2$ (self-resonance)

$$
L C=\frac{1}{\omega^{2}} \simeq 6.3 \times 10^{-10}
$$

Multi-layer self-resonance imposes:

$$
N_{2} a_{2} \simeq \frac{x}{20 h} \simeq 400
$$

We use: $\quad a_{2}=5 \mathrm{~m}$

$$
\begin{aligned}
& N_{2}=80 \\
& L_{2}=\mu_{0} N_{2}^{2} a_{2} f_{S}=.25 \text { hen } \\
& C_{2} \simeq .0025 \mu \mathrm{fd}
\end{aligned}
$$

Also $\quad Q_{2} \simeq 4 \pi$ (somewhat arbitrarily)

$$
\begin{aligned}
& k^{3}=\left(\frac{R}{d}\right)^{3} \simeq 3 \times 10^{-3} \\
& \ell=\sqrt{2} d \simeq 280 \mathrm{~m}
\end{aligned}
$$

Signal (Alluvium)

$$
\begin{aligned}
B_{c} & =\frac{\mu_{0} m_{1}}{2 \pi} \frac{1}{\left(a_{1}^{2}+d^{2}\right)^{3 / 2}} e^{-d / \delta} \\
m_{c} & =4 \pi R^{3} \frac{B_{c}}{\mu_{0}} \quad \sin \theta=\sqrt{2 / 3} \quad \cos \xi=1 \\
B_{S} & =\frac{\mu_{0} m_{c}}{4 \pi} \frac{\sin \theta}{r^{3}} e^{-r / \delta} \quad r=\sqrt{3} d \quad a_{1}=d \\
S & =\left(\pi a_{2}^{2} N_{2}\right) \omega B_{s} Q_{2} \cos \xi \quad r \\
& =\left(\pi a_{2}^{2} N_{2}\right) \omega\left(\frac{\mu_{0}}{4 \pi}\right)\left(\frac{\sin \theta}{r^{3}}\right) e^{-r / \delta}\left(\frac{4 \pi R^{3}}{\mu_{0}}\right)\left(\frac{\mu_{0} m_{1}}{2 \pi}\right) \frac{1}{\left(a_{1}^{2}+d^{2}\right)^{3 / 2}} e^{-d / \delta_{Q_{2}} \cos \xi} \\
& =\frac{1}{36}\left(\mu_{0} m_{1} \omega Q_{2} N_{2}\right) \frac{a_{2}^{2}}{d^{3}} k^{3} e^{-2.73}
\end{aligned}
$$




$$
\text { Find: } \quad \begin{aligned}
\quad & =\frac{1}{36}\left(4 \pi \times 10^{-7} \times 3 \times 10^{7} \times 2 \times 10^{4} \times 4 \pi \times 80\right) \frac{25}{8 \times 10^{6}} \times 10^{-3} \times \frac{1}{15} \\
& \simeq .004 \text { volt } \max
\end{aligned}
$$

In Granite $S \rightarrow .06$ volt $\max$

Background - This geometry has basically a very high order of background rejection. Assuming a truly symmetric generator field distribution (axial and equatorial), the background signal depends on the precision of sensor loop construction and axial alignment (in the equatorial plane of the generator 100p). If these geometrical criteria are satisfied, then the small radial field components threading "upper" and "lower". halves of the sensor loop produce cancelling constributions.

Perhaps the most significant higher-order background mechanism in the practical situation, would be that due to the distortion of the field lines due to eddy-current "drag" at an air-alluvium interface. We can estimate this by virtue of the characteristic relaxation time time of the medium: $\tau=\frac{\mathrm{Kg}^{\mathrm{K}}}{\sigma} \sim 3 \times 10^{-8} \mathrm{sec}$. The "drag" angle will be of order:

$$
\sin \psi \sim \cos \xi \sim \omega \tau=2 \times 10^{4} \times 3 \times 10^{-8}=6 \times 10^{-4} \text { radian }
$$

The equatorial field is:

$$
B_{b}=\left(\frac{\mu_{0}{ }_{1}}{4 \pi}\right) \frac{1}{\ell^{3}} \quad\left|\frac{\partial B_{b}}{\partial r}\right|=\frac{B_{b}}{3 \ell}
$$

and

$$
\begin{aligned}
B & \simeq\left(\pi a_{2}^{2} N_{2}\right) \omega\left(\frac{2}{3} a_{2} \frac{\partial B_{b}}{\partial r}\right) Q_{2} \cos \xi \\
& =\frac{1}{18}\left(\mu_{0} m_{1} \omega Q_{2} N_{2}\right) \frac{a_{2}^{3}}{l^{4}} \cos \xi
\end{aligned}
$$


This leads to: $S / B=2 e^{-2.73} \frac{l^{4}}{d^{3} a_{2}} \frac{k^{3}}{\cos \xi}=8 e^{-2.73} \frac{d}{a_{2}} \frac{k^{3}}{\cos \xi}$

$$
=\frac{8}{15} \times \frac{200}{5} \times \frac{10^{-3}}{6 \times 10^{-4}} \simeq \underline{40} \text { in alluvium }
$$

Even this can be improved by tipping the sensor "slightly" (.010!). Note that this $S / B$ is worse in granite, since $\tau \rightarrow 10^{-6}$, and $\cos \xi \rightarrow .02$.

Find

$$
S / B \simeq 20 \text { in granite }
$$

In itself, this is apparently not a serious problem in the VLF domain.

In order to evaluate the required precision of system symmetry, we calculate $N_{f}$ with respect to the maximum possible background contribution at $90^{\circ}$ sensor rotation:

$$
\mathrm{B}_{\mathrm{b}}=\frac{\mu_{0}{ }^{m} 1}{4 \pi} \frac{1}{\ell^{3}} \quad \ell=\sqrt{2} \mathrm{~d}
$$

and

$$
\begin{aligned}
B & \simeq\left(\pi a_{2}^{2} N_{2}\right) \omega B_{b} Q_{2} \\
& =\frac{3}{4}\left(\mu_{0} m_{1} \omega Q_{2} N_{2}\right) \frac{a_{2}^{2}}{l^{3}}
\end{aligned}
$$

Then

$$
\begin{aligned}
S / B & =\frac{1}{9}\left(\frac{l}{d}\right)^{3} k^{3} e^{-2.73} N_{f}=(2 \sqrt{2} / 9) e^{-2.73} k^{3} \\
& =2 \times 10^{-4} N_{f} \quad \text { and } \quad N_{f} \sim 5000 \text { in Alluvium }
\end{aligned}
$$

In Granite

$$
S / B \sim 3 \times 10^{-3} N_{f} \text { and } \quad \underline{N_{f} \sim 300}
$$

Evaluation. Inasmuch as the intrinsic geometric nulling furnishes at least a factor of 100 , I evaluate this scheme as being marginal from a technical standpoint. We will see that it is the only. VLF scheme which passes this test. However, on grounds of the scale of the hardware concept, it is programmatically very unattractive. 


\section{Axial Illumination, Concentric Loops}

In this singular scheme, the sensor loop is coaxial and coplanar with the generator loop, their mutual axis passing through the detonation point. The sensor loop is constructed as a quadrupole, so that one obtains first-order self-cancellation of the mutual-coupling background. By virtue of nulling coils, interlaced windings, etc., one hopes to achieve higher-order self-cancellation.

I assume the same generator element as was specified in the previous case. The sensor quadrupole will be considered here, as equivalent to two of the previous sensor loops, connected back-to-back. (It would most likely not be constructed that way.) The additional essential specification required by this case, is the effective sensor quadrupole separation. I take this as $s_{2}=5 \mathrm{~m}$.

Signal (Alluvium)

$$
\begin{aligned}
& B_{c}=\frac{\mu_{0} m_{1}}{2 \pi} \frac{1}{\left(a^{2}+d^{2}\right)^{3 / 2}} e^{-d / \delta} \\
& m_{c}=4 \pi R^{3} \frac{B_{c}}{\mu_{0}} \\
& B_{s}=\frac{\mu_{0} m_{c}}{4 \pi} \frac{\sin \theta}{d^{3}} e^{-d / \delta}
\end{aligned}
$$

Now

$$
S=\left(\pi a_{2}^{2} N_{2}\right) \omega B_{e f f} Q_{2} \cos \xi
$$

in which: $\quad B_{\text {eff }}=s_{2}\left|\frac{\partial B}{\partial r}\right|+s_{2}\left(\frac{3}{d}+\frac{1}{\delta}\right) B_{S}$ 
$-110-$

Then: $\begin{aligned} S & =\left(\pi a_{2}^{2} N_{2}\right) \omega S_{2}\left(\frac{3}{d}+\frac{l}{\delta}\right)\left(\frac{\mu_{0}}{4 \pi}\right)\left(\frac{\sin \theta}{d^{3}}\right) e^{-d / \delta}\left(\frac{4 \pi R^{3}}{\mu_{0}}\right) \frac{{ }_{0}{ }_{0}{ }_{1}}{2 \pi} \frac{1}{\left(a_{2}+d^{2}\right)^{3 / 2}} e^{-d / \delta} Q_{2} \cos \xi \\ & =\frac{1}{2}\left(\mu_{0} m_{1} \omega Q_{2} N_{2}\right) \frac{a_{2}^{2} s_{2}}{\left(a_{1}^{2}+d^{2}\right)^{3 / 2}}\left(\frac{3}{d}+\frac{1}{\delta}\right) k^{3} \sin \theta \cos \xi \mathrm{e}^{-2 d / \delta} .\end{aligned}$

Inasmuch as $\delta \sim d \sim a_{1}$ and $\sin \theta=\cos \xi \equiv 1$

$$
\begin{aligned}
S & =\left(\mu_{0} m_{1} \omega Q_{2} N_{2}\right)\left(\frac{a_{2}^{2} S_{2}}{2}\right) k^{3} e^{-2} \\
& =\left(4 \pi \times 10^{-7} \times 3 \times 10^{7} \times 2 \times 10^{4} \times 4 \pi \times 80\right)\left(\frac{25 \times 5}{\sqrt{2} \times 16 \times 10^{8}}\right) \times 10^{-3} \times \frac{1}{7} \\
& \simeq .006 \text { Volt max }
\end{aligned}
$$

In Granite: $S \rightarrow .04$ Volt $\max$

Background - In order to evaluate the severity of this problem we write the signal induced in half of the quadrupole:

$$
\text { and } \begin{aligned}
B & =\left(\pi a_{2}^{2} N_{2}\right)\left(\omega B_{0}\right) Q_{2} \\
& =\left(\frac{\mu_{0} m_{2}}{2 \pi} \mu_{0} m_{1} \omega Q_{2} N_{2}\right) \frac{a_{2}^{2}}{a_{1}^{3}}
\end{aligned}
$$

So $S / B=2 \frac{a_{1}^{3}}{a_{2}^{2}} \frac{a_{2}^{2} s_{2}}{\sqrt{2} d^{4}} k^{3} e^{-2} N_{f}=\sqrt{2} \frac{a_{1}^{3} s_{2}}{d^{4}} k^{3} e^{-2} N_{f}$

Since $a_{1}=d: \quad S / B \rightarrow \sqrt{2} \frac{S_{2}}{d} k^{3} e^{-2} N_{f}$

where $N_{f}$ is the ruling factor.

Then in Alluvium:

$$
S / B \sim \sqrt{2} \times \frac{5}{200} \times 10^{-3} \times \frac{1}{7} N_{f} \sim 5 \times 10^{-6} N_{f}
$$


Evidently for unity ratio, $\mathrm{N}_{\mathrm{f}} \sim 2 \times 10^{5}$. This is a major instrumentational challenge, even with the quadrupole concept, local field nulling, etc., to help us out.

In Granite $S / B \rightarrow 3 \times 10^{-5} \mathrm{~N}_{f}$ and $\quad \mathrm{N}_{f} \sim 3 \times 10^{4}$

Evaluation - The suppression requirement appears to be beyond the state-of-the-art. In addition, this scheme involves the most expensive hardware embodiment. I evaluate it as unfeasible on combined grounds (technical and programmatic).

\section{Equatorial Illumination, Orthogonal Loops ("Broadside")}

The "broadside" schemes suffer from the severe dimensional restriction on the generator loop (which is now vertical). The field equations intrinsically showed (thru the moment), that for given power/voltage limits, the $i l l$ umination depends strongly on loop radius:

Here I will use the same sensor loop specificiation as in the first case, but the generator loop needs to be redefined. The basic criteria remain the same, and $d=200 \mathrm{~m}$ still. Now take $a_{1} \sim 10 \mathrm{~m}$.

Inductance criterion becomes:

$$
N_{1}=\sqrt{\frac{L}{\mu_{0} a_{1} f_{S}}}=\sqrt{\frac{4 \times 10^{-3}}{4 \pi \times 10^{-7} \times 10 \times 6}} \simeq 7 \text { turns }
$$

and

$$
m_{1}=\pi a_{1}^{2} N_{1} I_{1}=\underline{3 \times 10^{5}} \text { which is much smaller. }
$$

A11 other problem numbers remain the same as the first case. 
Signal. For Alluvium:

$$
B_{c}=\frac{\mu_{0} m_{1}}{2 \pi^{2} a_{1}^{2} d}\left\{\frac{k}{1+x}-\frac{E}{1-x}\right\} e^{-d / \delta} \quad x=\frac{a_{1}}{d} \quad \text { etc. }
$$

which is here:

$$
\begin{aligned}
B_{c} & \simeq .01\left(\frac{\mu_{0} m}{\pi^{2} a_{1}^{2} d}\right) e^{-d / \delta} \\
m_{C} & =4 \pi R^{3} \frac{B_{c}}{\mu_{0}} \\
B_{S} & =\frac{\mu_{0} m_{c}}{4 \pi} \frac{\sin \theta}{r^{3}} e^{-r / \delta} \quad \sin \theta=\sqrt{2 / 3} \quad \cos \xi=1 \\
S & =\left(\pi a_{2}^{2} N_{2}\right) \omega B_{S} Q_{2} \cos \xi \quad r=\sqrt{3 / 2} d \\
& =\left(\pi a_{2}^{2} N_{2}\right) \omega\left(\frac{0}{4 \pi}\right)\left(\frac{\sin \theta}{r^{3}}\right) e^{-r / \delta}\left(\frac{4 \pi R^{3}}{\mu_{0}}\right) .01\left(\frac{\mu_{0} m_{1}}{\pi^{2} a_{1}^{2} d}\right) e^{-d / \delta} Q_{2} \cos \xi \\
& =\frac{.04}{9 \pi}\left(\mu_{0} m_{1} \omega Q_{2} N_{2}\right) \frac{a_{2}^{2}}{a_{1}^{2} d} k^{3} e^{-2.25}
\end{aligned}
$$

Find: $\quad S=\frac{.04}{9 \pi}\left(4 \pi \times 10^{-7} \times 3 \times 10^{5} \times 2 \times 10^{4} \times 4 \pi \times 80\right) \frac{25}{100 \times 200} \times 10^{-3} \times \frac{1}{9}$

In Granite: $\quad S+$ ol Volt $\max \quad \simeq 0075$ Volt $\max$

Background - In principle, this configuration also has a very high order of background rejection. Assuming a truly symmetric axial field, then the background signal depends on the extent to which the sensor loop can be made symetric with respect to a diameter. 
In order to evaluate the required precision of such symmetry, again we consider the maximum coupling case $\left(90^{\circ}\right.$ sensor rotation):

$$
\begin{aligned}
B_{b} & =\frac{\mu_{0} m_{1}}{2 \pi} \frac{1}{\left(a_{1}^{2}+\ell^{2}\right)^{3 / 2}} \\
B & =\left(\pi a_{2}^{2} N_{2}\right) \omega B_{b} Q_{2} \\
& =\frac{1}{2}\left(\mu_{0} m_{1} \omega Q_{2} N_{2}\right) \frac{a_{2}^{2}}{\left(a_{1}^{2}+\ell^{2}\right)^{3 / 2}} \quad\left(a_{1}^{2}+\ell^{2}\right)^{3 / 2} \sim \ell^{3}
\end{aligned}
$$

Then

$$
\begin{aligned}
S / B & \simeq \frac{.04}{18 \pi} \frac{l^{3}}{a_{1}^{2} d} k^{3} e^{-2.25} N_{f} \\
& =\left(\frac{.04}{18}\right) \times \frac{200^{3}}{(\sqrt{2})^{3} \times 10^{2} \times 200} \times 10^{-3} \times \frac{1}{9} N_{f} \\
& \sim 10^{-5} N_{f}
\end{aligned}
$$

And again for unity $S / B, N_{f} \sim 10^{5}$ in Alluvium.

In Granite, $S / B \rightarrow 10^{-4} N_{f}$ and $N_{f} \sim 10^{4}$.

These are very challenging numbers in terms of symmetry implications alone.

Evaluation - I evaluate this scheme as infeasible technically, being somewhat beyond the state-of-art. Programmatically, it would involve a relatively light penalty. 
The Radiation Schemes (Figure 6, typical)

These schemes involve the use of radiation-mode elements. They are applicable only to low-conductivity media, such as dry granite and basalt. The basic concept is to illuminate the cavity using a MF EM flux. The frequency criterion used here is $d>x_{g}>R_{c}$; we can then assume plane-wave illumination, with quasi-static coupling. The cavity communicates its history in the form of a re-radiated flux which would be observed by more-or-less conventional R.F. techniques.

As indicated before, there may be some severe "impedance matching" problems due to the air-granite interface, depending on the manner of illumination. The characteristic impedance above ground is $z_{0}=\sqrt{\frac{\mu_{0}}{k_{0}}}=377$ ohms of course; below ground, it is $z_{g} \simeq 126$ ohms. Thus the question of "burial" of elements is particularly salient to these schemes, inasmuch as the price of effective burial is also higher here. I have calculated these cases without taking account of the interface that is, as if the elements were imbedded in the granite.

There is one singular example, in which the cavity is 117 uminated by an axial field from a loop generator. The cavity field must then be described by the induction term, al though the observed "scattered" flux is calculated as radiative.

There are two "side illumination" radiative cases - one using a "smal1-loop" radiator, the other using a current dipole.

These estimates use a somewhat different approach, inasmuch as these various "simple" illumination schemes all lead to about the same cavity dipole strength. I have elected to complete the sensor part of the exercise using the "brightest" of those illuminators. 
I have not specified any particular background suppression schemes here, nor have I discussed more sophisticated possibilities using multielement arrays. In the MF domain, improvements latent in such schemes are large and comparatively well-known. Their application to shorterrange illumination and observation in granite should not encounter any basic difficulties, al though they may be somewhat expensive in practice. I "cut off" such further discussion mainly because it would involve new classes of selection and optimization rules, and because just the simple dipole examples presented here, appear to be relatively optimistic. Indeed, the problem becomes rather one of "selling granite" (as a test medium).

\section{Element Dimensions and Frequencies}

The Radiative Domain is defined essentially as the regime in which one encounters exterior cavity resonance. We saw that this would occur at $\sim 600 \mathrm{kHz}$ for our prototype cavity (at $R_{\max }$ ). We will perform the varicus radiation calculations at about half this frequency, so that the small dipole and quasistatic coupling approximations remain credible. The results will certainly be at least suggestive of the expectations throughout the domain.

Note that: $\quad x_{g}^{\prime}=\frac{7.3}{2 \pi} R_{\max } \sim 18 \mathrm{~W}^{1 / 3}$

Then at $5 \mathrm{kt}$ : $\quad x_{\mathrm{g}}^{\prime}=32 \mathrm{~m}$

The test case will be $\pi_{\mathrm{g}} \sim 50 \mathrm{~m} \ll \mathrm{d} \sim 200 \mathrm{~m}$.

and

$$
\omega \sim 2 \times 10^{6}
$$


In the "method of moments" adopted in these estimates, the precise element dimensions are unimportant. Evidently they should be comparatively small; where it matters, we will use $a=l=10 \mathrm{~m}$.

The "universal" path attenuation number is:

$$
e^{-d / \gamma} g=e^{-1.25} \simeq 1 / 3
$$

(This is actually conservative)

Transmitter Dipole Moments and Cavity Fields

We calculate the moments using the magnetic coupling mode, inasmuch as $\omega<\omega_{\text {res }}$. We assume that all power loss is radiative.

Sma11 Loop Generator:

$$
P=\frac{Z_{g}}{12 \pi x_{g}^{4}} m^{2}
$$

Thus

$$
m^{2}=\frac{12 \pi x_{g}^{4}}{Z_{g}}=2 \times 10^{11}
$$

Axial Induction Field:

$$
\begin{aligned}
B_{r} & \simeq \frac{\mu_{o}^{m}}{2 \pi} \frac{1}{x_{g}} \frac{\cos \theta}{d^{2}} e^{-d / \gamma} \\
& \simeq \frac{4 \pi \times 10^{-7} \times 4.5 \times 10^{5}}{2 \pi} \times \frac{1}{50} \times \frac{1}{(200)^{2}} \times \frac{1}{3} \\
& \simeq 1.5 \times 10^{-8} \mathrm{Web} / \mathrm{m}^{2} \text { peak }
\end{aligned}
$$


Equatorial Radiation Field:

$$
\begin{aligned}
B_{\theta} & \simeq \frac{\mu_{0} m}{4 \pi} \frac{1}{x_{g}^{2}} \frac{\sin \theta}{d} e^{-d / \gamma} \\
& \simeq \frac{4 \pi \times 10^{-7} \times 4.5 \times 10^{5}}{4 \pi} \frac{1}{(50)^{2}} \times \frac{1}{200} \times \frac{1}{3} \\
& =3 \times 10^{-8} \mathrm{Web} / \mathrm{m}^{2}
\end{aligned}
$$

Short Wire Generator

$$
\begin{aligned}
p & =\frac{z_{g}}{12 \pi x_{g}^{2}} p^{2} \\
\text { Thus } \quad p^{2} & =\frac{12 \pi x_{g}^{2}}{Z_{g}} \quad p^{2}=7.5 \times 10^{7} \quad p \sim 9 \times 10^{3} \text { (mks) }
\end{aligned}
$$

Equatorial Radiation. Field

$$
\begin{aligned}
B_{\phi} & =\frac{\mu_{0} p}{4 \pi} \frac{1}{x_{g}} \frac{\sin \theta}{d} e^{-d / \gamma} \\
& \sim \frac{4 \pi \times 10^{-7} \times 9 \times 10^{3}}{4 \pi} \times \frac{1}{50} \times \frac{1}{200} \times \frac{1}{3} \\
& =3 \times 10^{-8} \text { Web } / \mathrm{m}^{2}
\end{aligned}
$$

It looks like a good illumination number by any method is:

$\begin{array}{ll} & B=3 \times 10^{-8} \\ \text { Correspondingly } & E \sim 3 \mathrm{~V} / \mathrm{m}\end{array}$

Note that the displacement current density is almost as high as the conduction current density in the D.C. Alluvium case:

$$
J_{k}=k_{g} k_{o} \omega E \simeq 5 \times 10^{-4} \mathrm{amp} / \mathrm{m}^{2}
$$


$-118-$

Cavity Reradiation and Signals

Illumination: $\quad B_{O} \sim 3 \times 10^{-8}$ Web $/ \mathrm{m}^{2} \quad$ as obtained above

Cavity Moment: $\quad m_{C}=4 \pi R^{3} \frac{B_{0}}{\mu_{0}} \simeq 7 \times 10^{3} \quad \sin \theta=\frac{d}{\sqrt{\ell^{2}+d^{2}}}$

Signal Field: $\quad B_{S}=\frac{\mu_{0} m_{c}}{4 \pi} \frac{l}{x_{g}^{2}} \frac{\sin \theta}{\sqrt{\ell^{2}+d^{2}}} e^{-r / \gamma}$

and

$$
B_{S}=\left(\frac{\mu_{0}}{4 \pi}\right)\left(\frac{4 \pi}{\mu_{0}}\right) R^{3} B_{0} \frac{1}{x_{g}^{2}}\left(\frac{d}{\ell^{2}+d^{2}}\right) e^{-r / \gamma}=B_{0} \frac{d^{4}}{\left(\ell^{2}+d^{2}\right) x_{g}^{2}} k^{3} e^{-2} .
$$

We assume $\ell \sim d=200 \mathrm{~m}$.

$$
\begin{array}{ll}
B_{S} & =3 \times 10^{-8} \frac{(200)^{4}}{2 \times(200)^{2} \times(50)^{2}} \times 10^{-3} \times \frac{1}{7} \sim 3 \times 10^{-11} \text { Web } / \mathrm{m}^{2} \text { peak } \\
\text { Correspondingly } & E_{S} \simeq .003 \mathrm{~V} / \mathrm{m}
\end{array}
$$

The reradiate power is interesting:

$$
P=\frac{Z g}{12 \pi x_{g}^{4}} m^{2}=25 \text { watts : }
$$

Signal: First we establish what's permissible in loop geometry. Here we must use the "open coil" criterion:

$$
\begin{aligned}
N_{2} a_{2}^{2} & \simeq \frac{x_{0}^{2}}{300 h^{2}} \frac{(150)^{2}}{300 \times(3)^{2}} \simeq 10 \\
S & =\left(\pi N_{2} a_{2}^{2}\right) \omega B_{S} Q_{2} \cos \xi \\
& =(\pi \times 10) \times 2 \times 10^{6} \times 3 \times 10^{-11} \times 10^{2} \times .7 \simeq .15 \text { volt }
\end{aligned}
$$$$
\text { Then finally: } \quad S=\left(\pi N_{2} a_{2}^{2}\right) \omega B_{S} Q_{2} \cos \xi
$$ 
Background - We estimate this as an axial induction field, obtaining a suppression factor $N_{f}$, as for the previous examples:

$$
B_{r} \simeq \frac{\mu_{0} m}{2 \pi} \cdot \frac{1}{x_{g}} \frac{1}{l^{2}}=\frac{\mu_{0} m}{4 \pi} \frac{1}{x_{g}} \frac{1}{d^{2}}
$$

The signal field expression is:

$$
B_{\theta} \simeq \frac{\mu_{0}^{m}}{4 \pi} \frac{d^{3}}{x_{g}^{4}\left(\ell^{2}+d^{2}\right)} k^{3} e^{-3}
$$

So that: $\quad S / B=\left(\frac{d}{x_{g}}\right)^{3}\left(\frac{d^{2}}{l^{2}+d^{2}}\right) k^{3} e^{-3} N_{f}$

which is $\quad=4^{3}\left(\frac{(200)^{2}}{2 \times(200)^{2}}\right) \times \frac{10^{-3}}{20} N_{f}=1.5 \times 10^{-3} N_{f}$

(without implicit nulling)

So it looks like one does not have to work very hard at suppression here:

$$
\underline{N_{f}} \sim 600
$$

Evaluation - Some form of radiation scheme would appear to be feasible in Granite. The hardware requi rements are (comparatively) modest. However, the Test program implications of "moving to Granite" are extensive and probably prohibitive. 


\section{TABLE I - FEASIBILITY STUDY CONTENTS}

Introduction

Vocabulary

Summary Tables

THE EXPERIMENTAL CONDITIONS

The Prototype Explosion

The Earth Medium

The Air-Earth Interface

The Plasma Cavity Model

Cavity Growth

Strong Shock

Cracking

CONFIGURATION CRITERIA \& CONSTRAINTS

Frequency Domain Considerations

"Evolution"; Domain Choice Factors:

a. Power Frequency

b. EM MFP

c. Quasistatic vs. Plane-Wave Approximations

d. Information Bandwidth

e. Cavity Resonance

Generator Coupling

Adiabatic Equilibrium Assumption

Displacement Criterion

Field Curvature

Quasistatic Approx.

Plane-Wave Approx.

Sensor Configurations

Resonance

Phase (or "Polarity")

Polarization (or Orientation)

Location

Symmetry 


\section{ANALYSIS DESCRIPTIONS}

Some Dimensional Relations; The Prototype Test

Power-Voltage Considerations

Conduction Current Schemes

Laplace Correspondence

Earth Current Generators

Field \& Current Distributions

The Immersed Spherical Conductor

Quasistatic Magnetic Fields

Moments Defined; Diamagnetic Sphere

Dipole Equations; Current \& Magnetic Dipoles

The Quasistatic Limit

Exact Loop Expressions

VLF Hardware Aspects

Transmitter: Generator Loop Design

\section{Resonance \& Q}

Power Req't - Earth-coupled case

The Optimum Illumination Problem

Receiver: Sens.or Loop Design

Loop Signals; Earth Probe Signals

Bandwidth Criteria

The Optimum Coupling Problem

Radiative Field Descriptions

Radiative Dipole Expressions

Attenuation

Power Req't \& Radiation Resistance

Induced Current; Cavity Moments 
THE PROTOTYPE CASES

The Conduction Schemes

D.C. Conduction Case; Noise

VLF Conduction; Signal/Background

Quasistatic Magnetic Field Schemes

Axial Illum., Orthogonal loops

Axial Illum., Concentric loops

Equatorial Illum., Orthogonal loops

The Radiation Schemes

Element Dimensions, Frequencies

Transmitter Moments \& Cavity Fields

Cavity Reradiation \& Signals 
TABLE II - FEASIBILITY STUDY SUMMARY

Basic Assumptions: Source Power: $100 \mathrm{~kW}$

Source Voltage: $10 \mathrm{kV}$

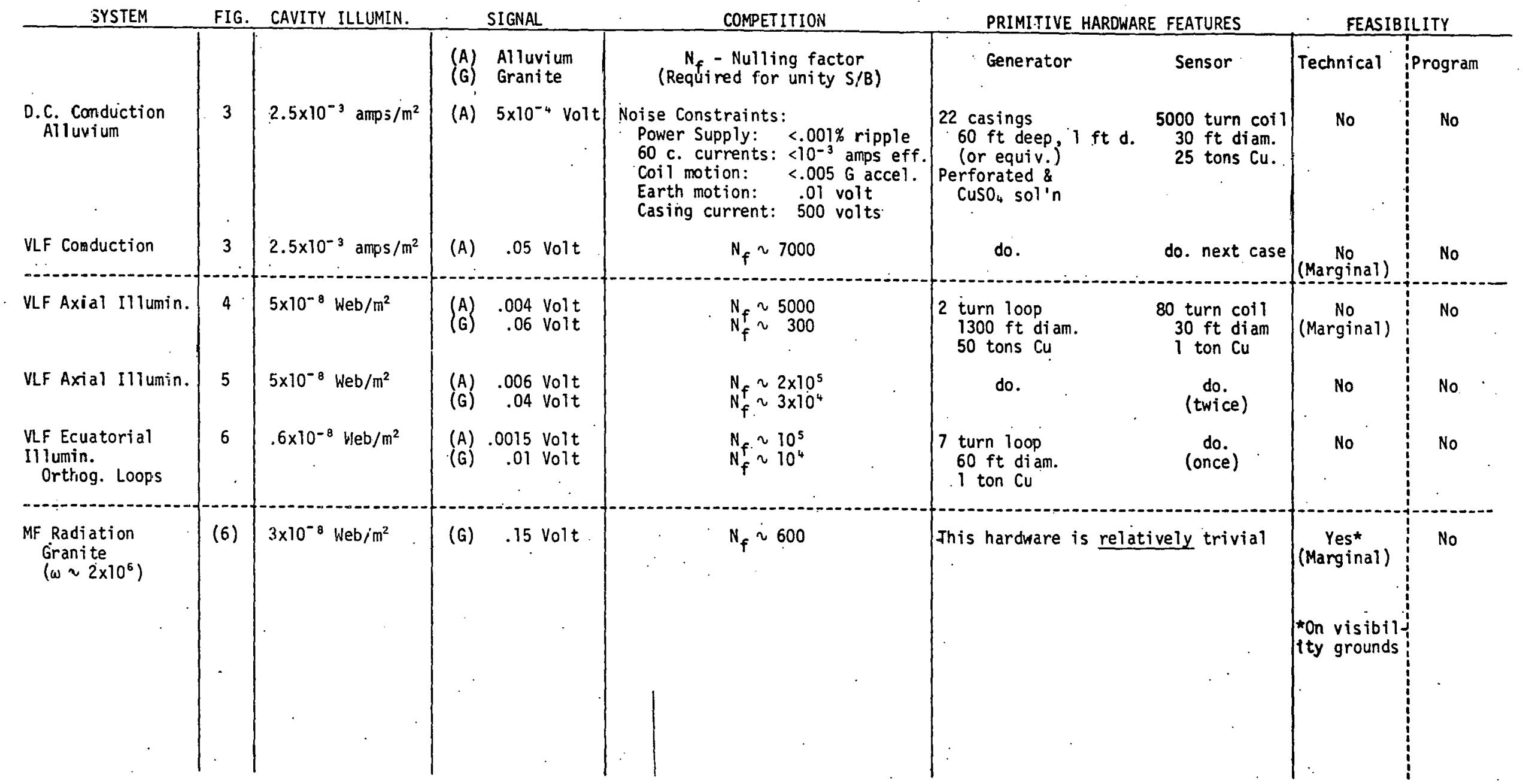




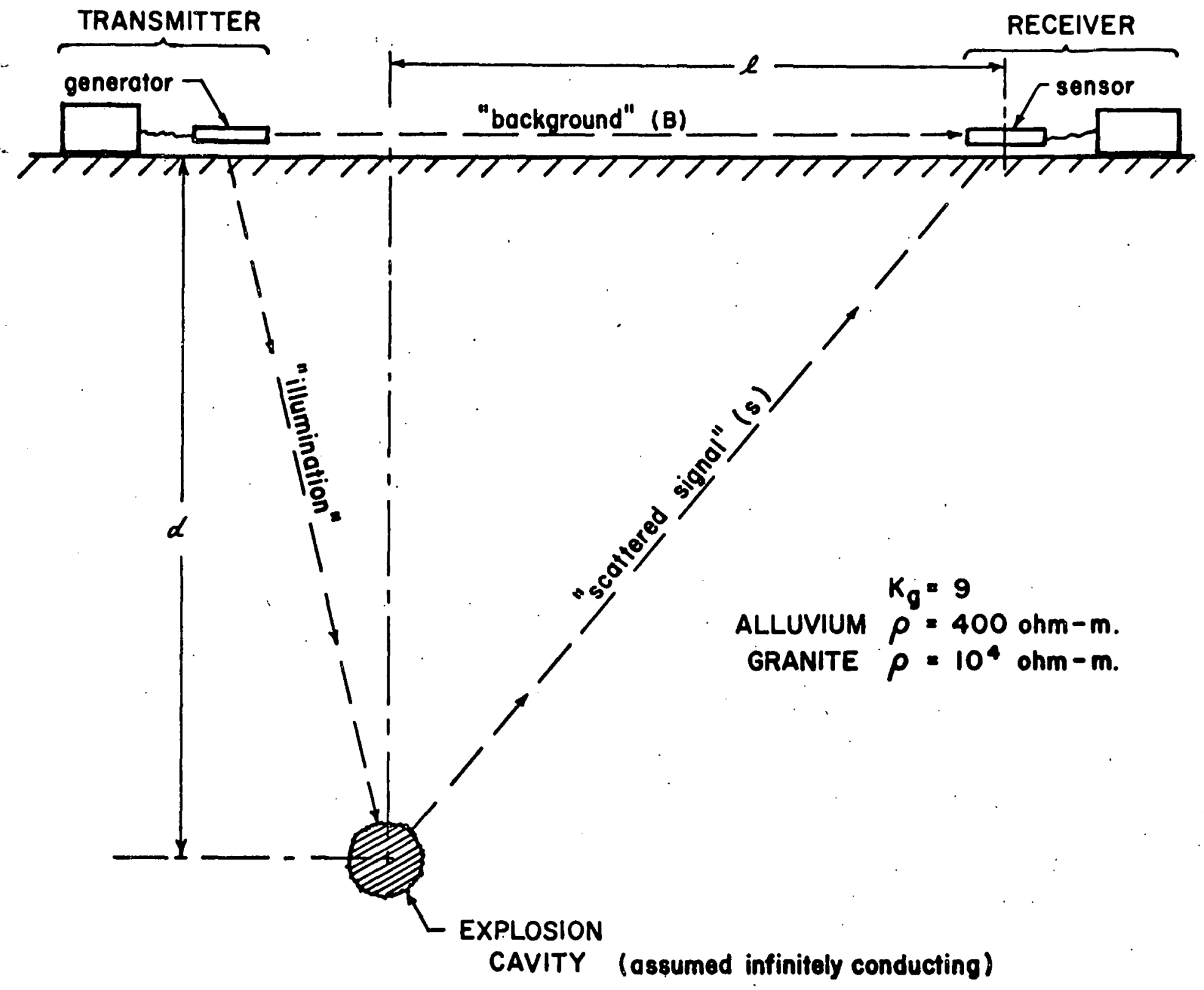

Fig I THE BASIC CONCEPT 


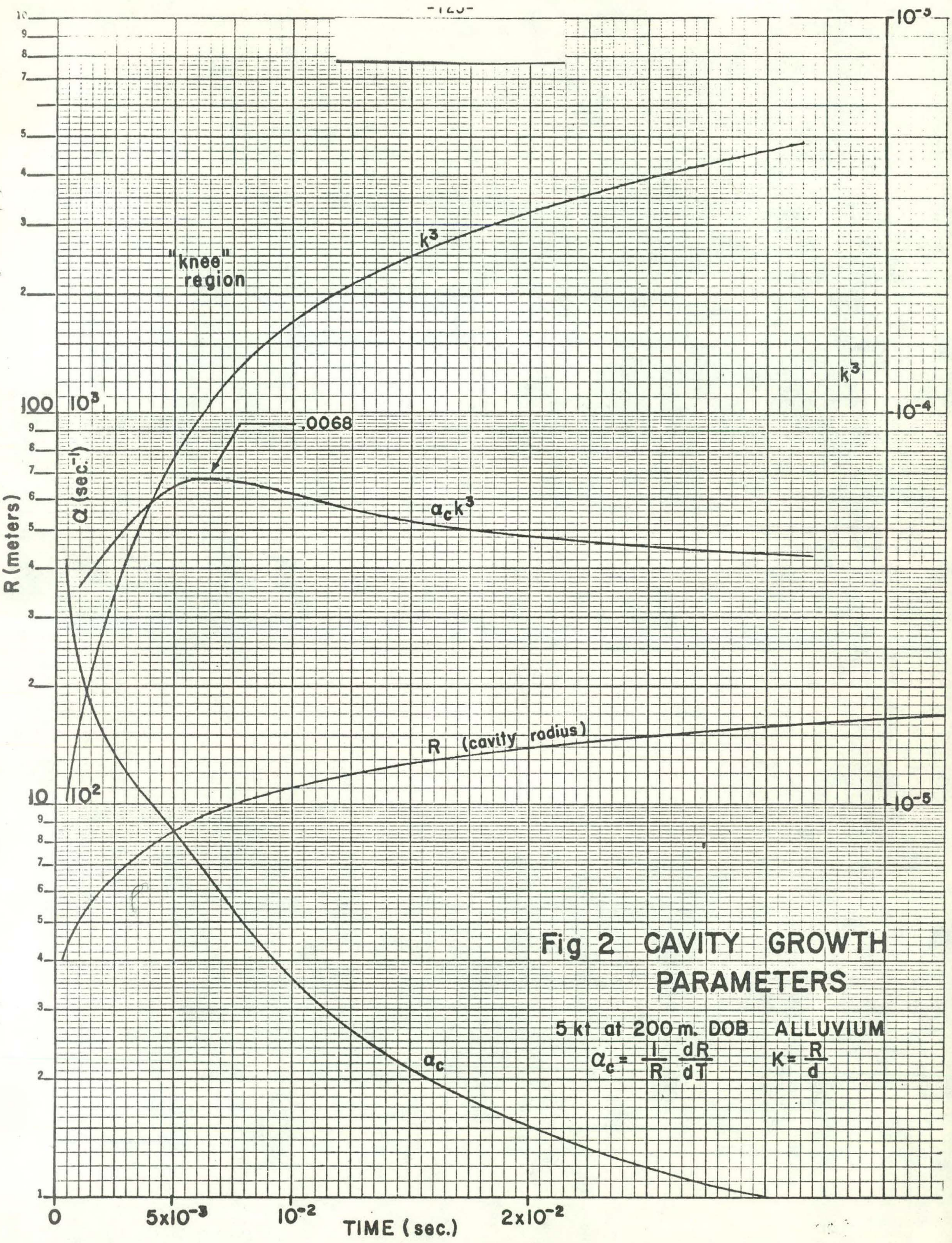




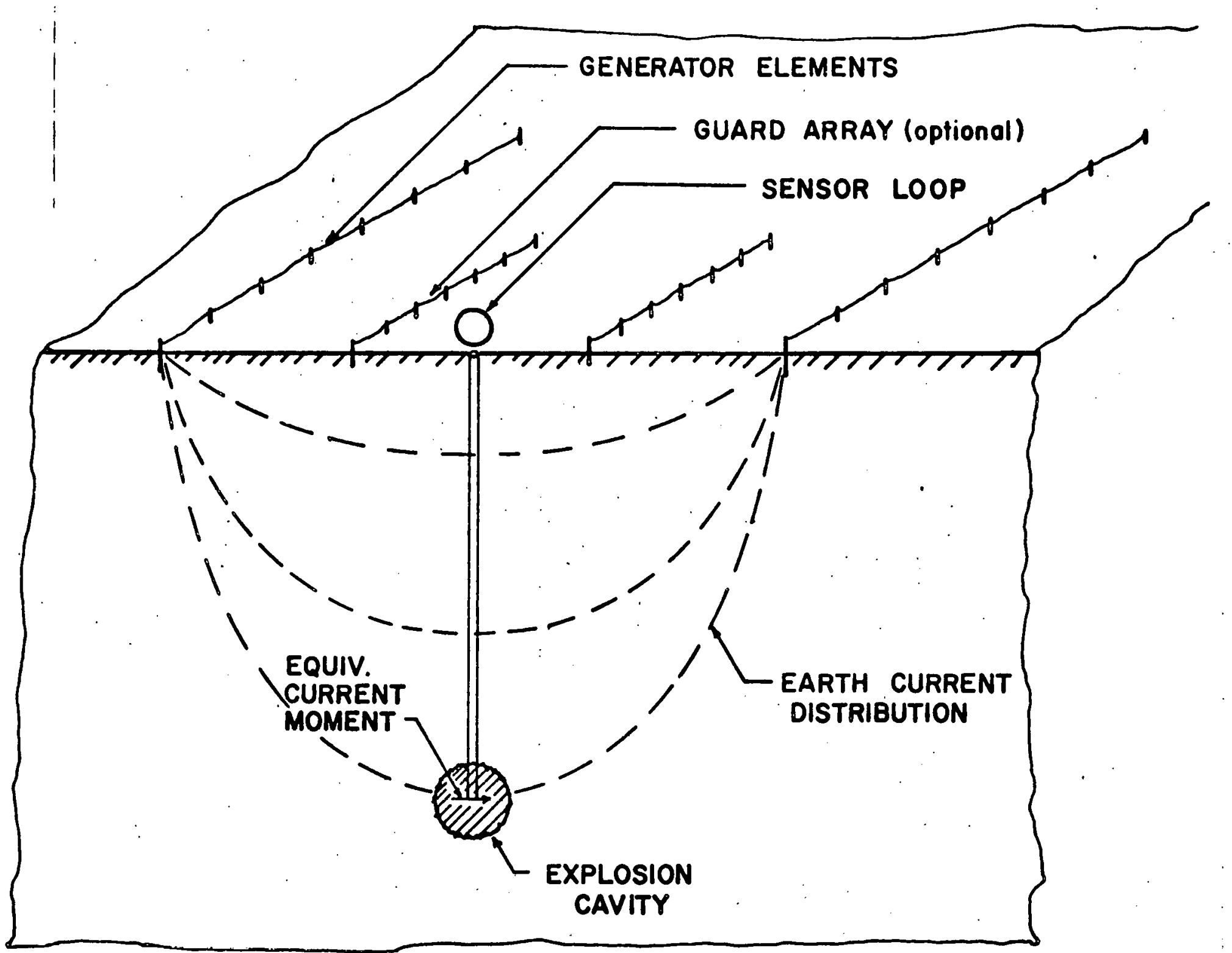

Fig 3 EARTH CONDUCTION SCHEMES IN ALLUVIUM (DC \& VLF) 


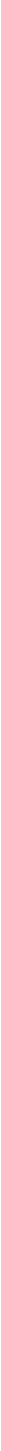

Fig 4 VLF AXIAL ILLUMINATION, CROSSED LOOPS 


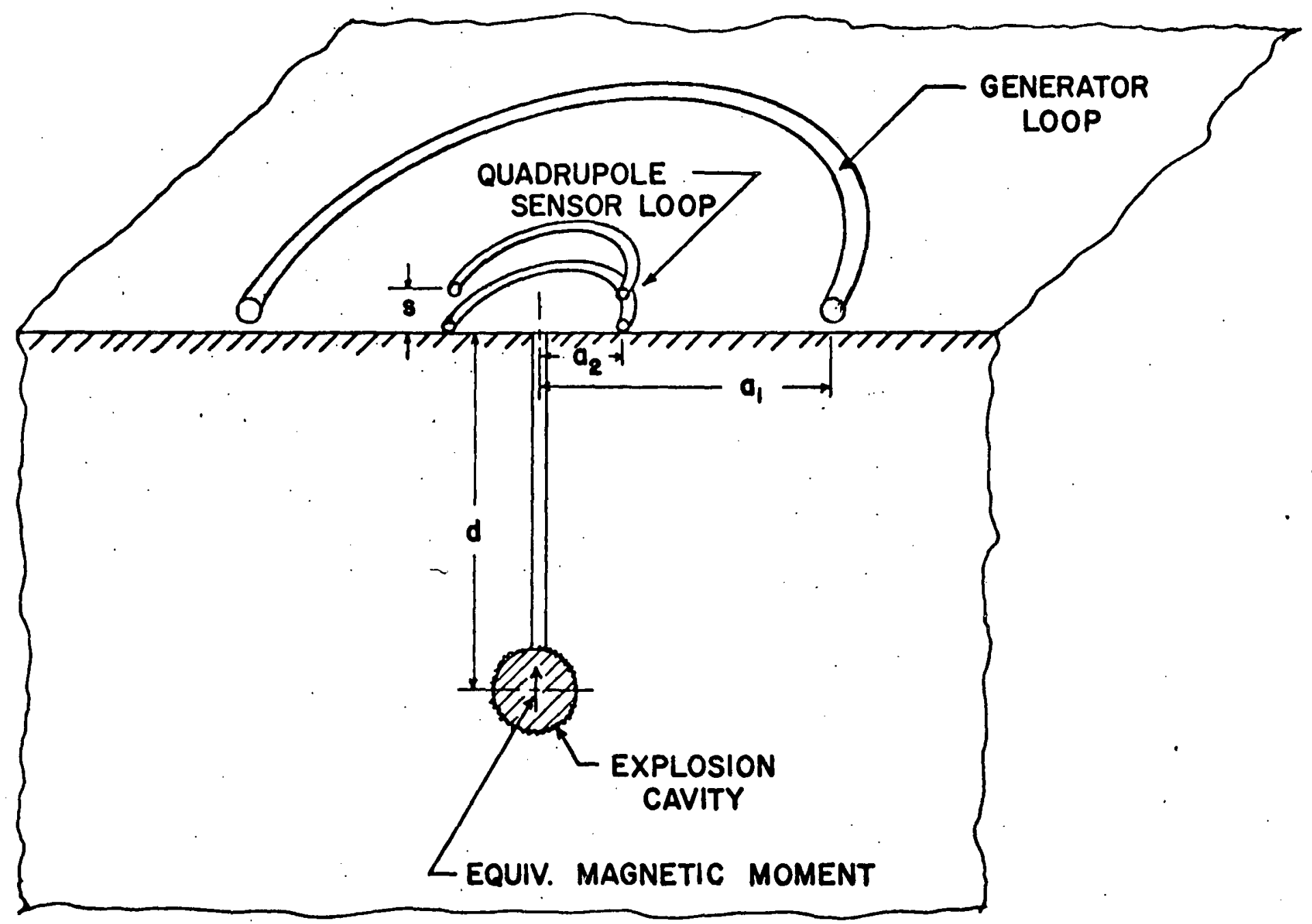

Fig 5 VLF AXIAL ILLUMINATION QUADRUPOLE SENSOR 


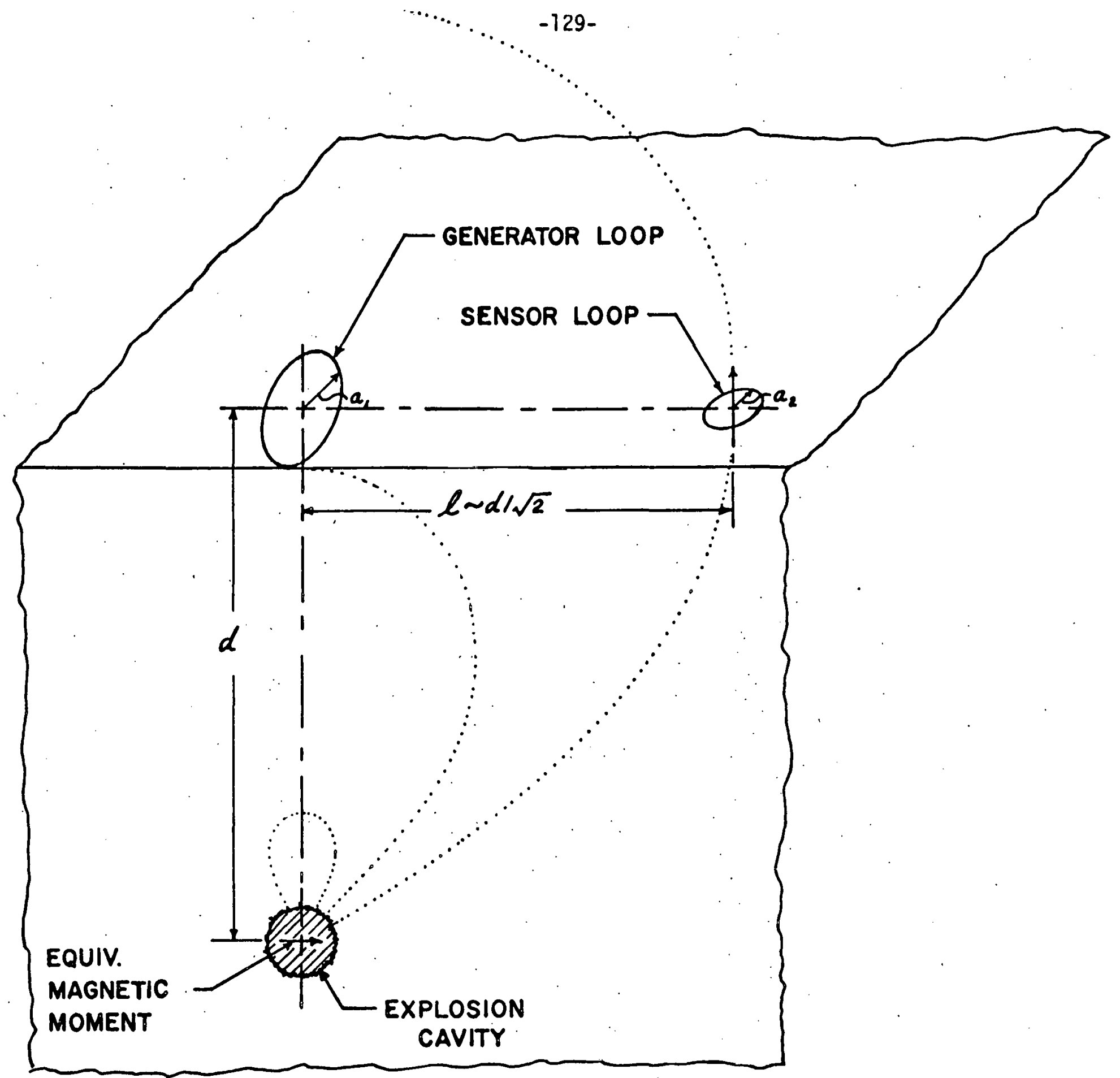

Fig 6 VLF EQUATORIAL ILLUMINATION CROSSED LOOPS 


\section{Distribution}

T.I.D. (15)

L. F. Wouters (6)

External:

R. H. Beers/J. J. Walker, EG\&G, Los Alamos, NM TIC, Oak Ridge, TN (27).

NOTICE

"This report was prepared as an account of work sponsored by the United States Government. Neither the United States nor the United States Energy Research \& Development Administration, nor any of their employees, nor any of their contractors, subcontractors, or their employees, makes any warranty, express or implied, or assumes any legal liability or responsibility for the accuracy, completeness or usefulness of any information, apparatus, product or process disclosed, or represents that its use would not infringe privately-owned rights."

Printed in the United States of America Available from

National Technical Information Service

U.S. Department of Commerce 5285 Port Royal Road

Springfield, Virginia 22151

Price: Printed Copy $\$ \quad *$; Microfiche $\$ 2.25$

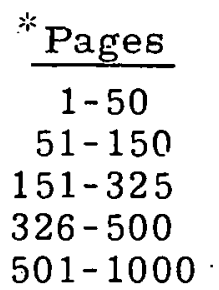

NTIS

Selling Price

$\$ 4.00$

$\$ 5.45$

$\$ 7.60$

$\$ 10.60$

$\$ 13.60$ 